\title{
CHAPTER 5 BURNING PLASMA STUDIES AT JET
}

\author{
S. E. SHARAPOV, ${ }^{\text {* }}$ L.-G. ERIKSSON, ${ }^{\mathrm{b}}$ A. FASOLI, ${ }^{\mathrm{c}}$ G. GORINI, ${ }^{\mathrm{d}}$ J. KÄLLNE, ${ }^{\mathrm{e}}$ V. G. KIPTILY, ${ }^{\mathrm{a}}$ \\ A. A. KOROTKOV, ${ }^{a}$ A. MURARI, ${ }^{f}$ S. D. PINCHES,${ }^{a}$ D. S. TESTA,,${ }^{c}$ and P. R. THOMAS ${ }^{b}$ \\ ${ }^{a}$ Euratom/UKAEA Fusion Association, Culham Science Centre, Abingdon, Oxfordshire OX14 3DB, United Kingdom \\ ${ }^{\mathrm{b}}$ Association Euratom-CEA, CEA/DSM/DRFC, CEA-Cadarache, France \\ ${ }^{\mathrm{C}}$ CRPP/EPFL, Association EURATOM-Confédération Suisse, Lausanne, Switzerland \\ distituto di Fisica del Plasma, EURATOM-ENEA-CNR Association, Milan, Italy \\ e INF, Uppsala University, Association Euratom-VR, Uppsala, Sweden \\ ${ }^{\mathrm{f}}$ Consorzio RFX-Associazione Euratom-ENEA sulla Fusione, Padova, Italy
}

Received June 22, 2007

Accepted for Publication August 3, 2007

Studies establishing key phenomena and developing diagnostics for energetic particle physics, which are essential for the next step burning plasma experiments such as the International Thermonuclear Experimental Reactor (ITER), have been performed at the Joint European Torus (JET). Experiments have demonstrated clear selfheating of deuterium-tritium (D-T) plasma by alpha particles as a maximum in electron temperature at an optimum mixture of $60 \pm 20 \%$ tritium. The change in electron temperature produced by alpha heating, $\Delta T_{e}(0)=1.3 \pm$ $0.23 \mathrm{keV}$, was as expected from classical heating, whereas the heating of thermal ions was higher than expected from reference deuterium discharges. Alfvén eigenmodes were stable in the highest fusion performance D-T plasmas, in agreement with the modeling. Systematic studies on the existence and properties of Alfvén eigenmodes with external antenna driving and detecting Alfvén eigenmodes are presented. The formation of fuel ion tails due to alpha-particle knock-on effects is described as derived from neutral particle analyzer and neutron emission spectrometry in D-T experiments. The gamma-ray diagnostics are shown to measure profiles and energy distribution functions of high-energy ions and alpha particles. Time- and space-resolved gamma-ray images demonstrated for the first time the possibility of measuring several types of energetic ions simultaneously. The novel technique of detecting unstable Alfvén eigenmodes with interferometry is found to be superior in detecting corelocalized Alfvén eigenmodes.

KEYWORDS: alpha particles, Alfvén eigenmodes, tokamak Note: Some figures in this paper are in color only in the electronic version.

\section{Contents-Chapter 5}

I. INTRODUCTION

II. OBSERVATION OF ALPHA HEATING IN JET D-T PLASMAS

II.A. Alpha-Particle Heating of Electrons

II.B. Alpha-Particle Heating of Bulk Ions

II.C. Summary

III. ENERGETIC PARTICLE-DRIVEN AEs IN JET D-T PLASMAS

III.A. Instability Mechanisms and Linear AEs

III.B. Stability Diagram for Alpha-Particle-Driven AEs in Hot-Ion H-Mode

III.C. AEs in High-Fusion Power-Optimized Shear D-T Plasmas in JET

*E-mail: sergei.sharapov@jet.uk
III.D. AE Observation in the Afterglow Phase of Optimized Shear Discharges

III.E. Conclusions

IV. ACTIVE STUDIES OF AES USING EXTERNAL ANTENNAS

IV.A. Experimental Setup

IV.B. Demonstration of the Existence of Stable, Weakly Damped AEs

IV.C. Damping Rate of Global AEs as a Function of the Plasma Shape

IV.D. Damping Rate of Global AEs as a Function of Plasma Parameters

IV.E. Conclusions and Outlook

V. DEVELOPING NEW DIAGNOSTIC TECHNIQUES FOR BURNING PLASMA

V.A. Measurements of the Distribution Function of Energetic Ions with a Neutral Particle Analyzer

V.B. Information on the Confined Alpha-Particle Population in the Neutron Emission Spectrum 
V.C. Gamma-Ray Spectroscopy and Gamma-Ray Tomography in JET Plasmas with ICRH-Accelerated Ions

V.D. First Gamma-Ray Measurements of Fusion-Born Alpha Particles in Trace Tritium Experiments in JET

V.E. Interferometry/Reflectometry Detection of Fast IonDriven Alfvén Cascade Eigenmodes in Reversed Shear JET Discharges

V.F. Simultaneous Time-Resolved Measurements of ICRHAccelerated ${ }^{3} \mathrm{He}$ Ions with $E>500 \mathrm{keV}$ and Coupled Energetic Ion-Driven AEs

V.G. Further Improvements of Fast Ion Diagnostics in JET VI. CONCLUSIONS AND OUTLOOK

REFERENCES

\section{INTRODUCTION}

Experimental study of thermonuclear burning plasmas on ITER, in which the power of plasma self-heating by fusion-born alpha particles exceeds auxiliary heating power, is an essential next step for magnetic nuclear fusion. ${ }^{1}$ To meet the requirements anticipated for this next step, present-day tokamaks worldwide study in depth the physics of energetic particles, which is a key issue for the burning plasmas. The JET tokamak ${ }^{2}$ is one of the best present-day machines for performing burning plasma studies since JET has the D-T capability for performing experiments with fusion-born alpha particles and, historically, the JET tokamak was designed and built to "operate in conditions where alpha particles are produced and confined" in D-T plasmas. ${ }^{3}$ Typical values of the plasma currents, $I_{p} \cong 3$ to $4.5 \mathrm{MA}$, used in JET highperformance operations are high enough for the first orbit losses of ions with energies in the $\mathrm{MeV}$ range to be negligible. The toroidal field ripples in JET are very small, $\delta \equiv\left(B_{\max }-B_{\min }\right) /\left(B_{\max }+B_{\min }\right) \cong 1 \%$ at the edge, and do not cause any significant losses of energetic ions either. ${ }^{4}$ Furthermore, the experimentally measured nonclassical (fluctuation-induced) diffusion coefficient for ions accelerated by ion cyclotron resonance heating (ICRH) was estimated in JET to be $D \leq 0.2 \mathrm{~m}^{2} / \mathrm{s}$ only, ${ }^{5}$ except during magnetohydrodynamic (MHD) events such as sawteeth or when the wave-particle resonance becomes possible, as in the case of Alfvén eigenmodes (AEs). This value of the energetic ion transport is much smaller than the thermal plasma transport, $D \geq 1 \mathrm{~m}^{2} / \mathrm{s}$. One concludes that the JET tokamak is well suited for studies of energetic ions since these are well confined within the plasma.

The good confinement of energetic ions and thermal plasma in JET makes it possible to generate and sustain significant populations of ions with energies significantly higher than plasma temperature and with the energy content up to that of the thermal plasma. Energetic ions in JET are produced with neutral beam injection (NBI) and ICRH techniques capable of accelerating hydrogen isotopes $\mathrm{H}, \mathrm{D}, \mathrm{T}$, and ${ }^{3} \mathrm{He}$ up to the $\mathrm{MeV}$ energy range.$^{6-8}$ A population of energetic ${ }^{4} \mathrm{He}$ ions can be obtained in D-T fusion reactions with quite significant val- ues of the fast ion density and energy contents. ${ }^{9,10}$ It is also possible to accelerate a population of ${ }^{4} \mathrm{He}$ ions up to the $\mathrm{MeV}$ energy range with NBI plus ICRH technique in radiation-free helium plasma. ${ }^{11}$

Among the techniques of plasma heating, NBI heating with a power in excess of $20 \mathrm{MW}$ plays a major role in JET (Ref. 12). All high-performance scenarios rely on an NBI-produced fast ion population as the principal source of plasma heating. ${ }^{10}$ The NBI system in JET consists of two sources, which in the case of deuterium beams have nominal energy $E_{1}^{\mathrm{D}}=70$ to $80 \mathrm{keV}$ (octant 4, total power up to $P_{1}^{\mathrm{D}}=13.6 \mathrm{MW}$ ) and $E_{2}^{\mathrm{D}}=130$ to $140 \mathrm{keV}$ (octant 8 , total power up to $P_{2}^{\mathrm{D}}=7.6 \mathrm{MW}$ ). Both octants have beams with a tangency radius $1.3 \mathrm{~m}$ (normal) and $1.85 \mathrm{~m}$ (tangential). Apart from deuterium, both beams can inject hydrogen, helium, and ${ }^{3} \mathrm{He}$. A high-voltage NBI source in octant 8 was also used for injecting tritium beam with up to $E_{2}^{\mathrm{T}}=160 \mathrm{keV}$ and $P_{2}^{\mathrm{T}}=10.5 \mathrm{MW}$ in high-fusion power D-T experiments. Hydrogen gases produce neutral beams in three fractions at $E, E / 2$, and $E / 3$ energies, while helium beams are produced at energy $E$ only.

NBI heating in JET is ideally suited for providing heating of bulk ions since the injection energy of the beams is usually below the critical energy associated with the typical high electron temperature in JET. With high-power NBI, high-performance plasmas with bulk ion temperatures higher than the electron temperatures, $T_{i} \cong 2 T_{e}$, are usually obtained. In addition to heating, NBI is also providing fueling. In particular, tritium NBI at energy of $160 \mathrm{keV}$ was very effective in penetrating to the plasma core in hot-ion $\mathrm{H}$ modes, thus providing tritium fueling close to the optimum $\mathrm{D}: \mathrm{T}=50: 50$ mixture in high-fusion power D-T experiments in JET (Ref. 10).

The ICRH plant in JET is very flexible and allows a variety of ICRH schemes to be employed..$^{6-8,11,13}$ ICRH is used for heating of both electrons and ions, depending on the ratio between the critical energy and the tail temperature of ICRH-accelerated ions. In this chapter, only ICRH experiments generating temperatures of energetic ion tail in the $\mathrm{MeV}$ energy range are considered, since these ions can mimic fusion ions of a burning plasma. A more complete description of ICRH techniques used for achieving highest D-T neutron yields and for the mode conversion can be found in Ref. 8 in this special issue. Table I shows a comparison of some characteristic parameters of energetic ions in the $\mathrm{MeV}$ energy range obtained in high-fusion D-T experiments in JET and with different ICRH and ICRH plus NBI acceleration techniques versus ITER parameters.

In the classical scheme of plasma heating by fast ions, the fast ions transfer their energy to the thermal ions and electrons by Coulomb collisions. If the energy of the fast ions is less than a critical value, power flows mainly to thermal ions rather than to electrons (see, e.g., Ref. 14 and references therein). The critical energy at which the power to the electrons equals that to the ions is given by 
TABLE I

Characteristics of ICRH-Accelerated Ions and Fusion-Born Alpha Particles in JET Experiments*

\begin{tabular}{|c|c|c|c|c|c|}
\hline \multirow[b]{2}{*}{ References } & \multicolumn{4}{|c|}{ JET } & \multirow{2}{*}{$\frac{\text { ITER }}{1}$} \\
\hline & 6 and 7 & 6 and 7 & 11 & 1 and 9 & \\
\hline Type of fast ions & Hydrogen & ${ }^{3} \mathrm{He}$ & ${ }^{4} \mathrm{He}$ & Alpha & Alpha \\
\hline Source & ICRH tail & ICRH tail & ICRH tail & Fusion & Fusion \\
\hline Mechanism & Minority & Minority & $\begin{array}{l}\text { Third harmonic } \\
\text { of NBI }\end{array}$ & D-T nuclear & D-T nuclear \\
\hline Slowing down time, $\tau_{S}(\mathrm{~s})$ & 1.0 & 0.9 & 0.4 & 1.0 & 0.8 \\
\hline $\begin{array}{l}\text { Heating power per volume at the magnetic axis, } \\
P_{f}(0)\left(\mathrm{MW} / \mathrm{m}^{3}\right)\end{array}$ & 0.8 & 1.0 & 0.5 & 0.12 & 0.55 \\
\hline $\begin{array}{l}\text { Ratio of the on-axis fast ion density to electron } \\
\text { density, } n_{f}(0) / n_{e}(0)(\%)\end{array}$ & 1.0 & 1.5 & 1.5 & 0.44 & 0.85 \\
\hline On-axis fast ion beta, $\beta_{f}(0)(\%)$ & 2 & 2 & 3 & 0.7 & 1.2 \\
\hline Volume-averaged fast ion beta, $\left\langle\beta_{f}\right\rangle(\%)$ & 0.25 & 0.3 & 0.3 & 0.12 & 0.3 \\
\hline $\begin{array}{l}\text { Normalized radial gradient of fast ion beta, } \\
\qquad \max \left|R \nabla \beta_{f}\right|(\%)\end{array}$ & $\approx 5$ & $\approx 5$ & 5 & 3.5 & 3.8 \\
\hline
\end{tabular}

*Predicted values of similar parameters are also given for alpha particles in ITER.

$$
E_{c r i t}=14.8 A_{f} T_{e}\left(\sum_{i} n_{i} Z_{i}^{2} / n_{e} A_{i}\right)^{2 / 3}
$$

where

$$
\begin{aligned}
A_{f} & =\text { atomic mass of the fast ions } \\
A_{i} & =\text { atomic mass of the thermal ions } \\
T_{e} & =\text { electron temperature } \\
n_{i} & =\text { ion density } \\
n_{e} & =\text { electron density } \\
Z_{i} & =\text { atomic number of the thermal ions. }
\end{aligned}
$$

The amount of energy that goes from ions with initial energy $E$ into the plasma ions is given by ${ }^{14}$

$$
G_{i}=\frac{E_{c r i t}}{E} \int_{0}^{E / E_{c r i t}} \frac{d y}{1+y^{3 / 2}},
$$

and this function $G_{i}\left(E / E_{c r i t}\right)$ is illustrated in Fig. 1. In accordance with Eqs. (1) and (2), the classical plasma heating by fast ions in JET plasmas with a D-T mixture $\mathrm{D}: \mathrm{T}=50: 50$ can be classified as shown in Table II.

It is of paramount importance for burning plasma studies to assess experimentally whether D-T plasma heating by fusion-born alpha particles is classical, so that one may rely upon the heat fluxes to electrons and ions used for predictions of plasma performance in a fusion reactor. The good confinement of the energetic ions has made feasible investigating effective D-T plasma self-heating by the fusion-born alpha particles during high-power D-T experiments in JET. The first observation of measurable alpha-particle heating was performed on TFTR with a

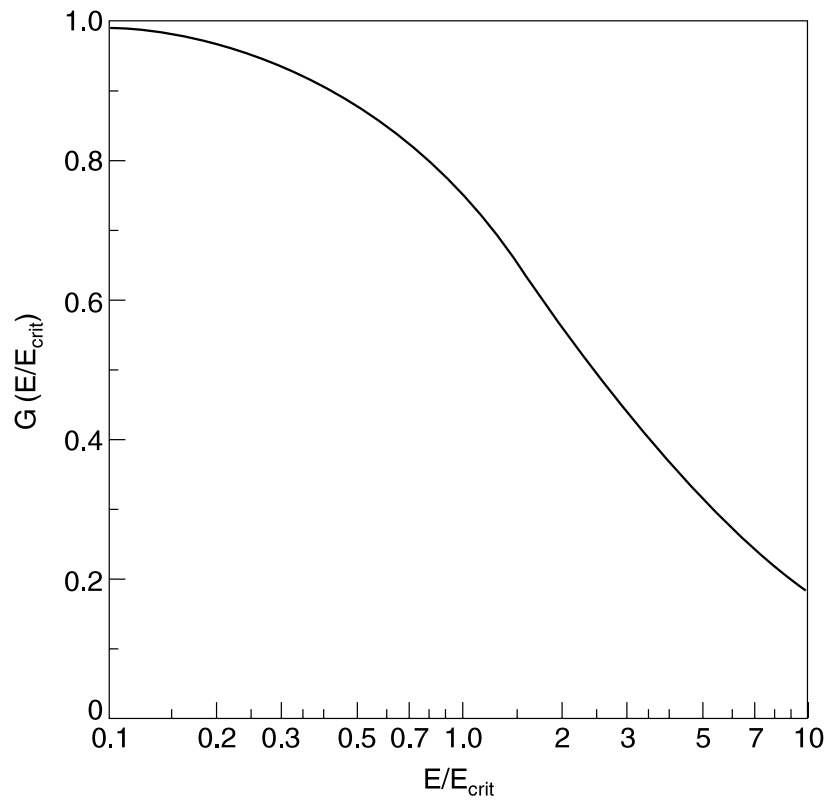

Fig. 1. Graph of the function $G\left(E / E_{c r i t}\right)$ given by formula (2) from Stix. ${ }^{14}$

fusion gain up to $Q \equiv P_{\text {fus }} / P_{\text {in }} \cong 0.27$, where $P_{\text {fus }}$ is the fusion power and $P_{i n}$ is the total input power to the machine. ${ }^{15}$ More favorable conditions for alpha-particle heating existed in the JET hot-ion H-mode plasmas with a significantly higher fusion gain, ${ }^{16} Q \cong 0.65$. It was nevertheless a challenging problem for JET to demonstrate clearly the alpha heating effect, and a specifically designed set of experiments was performed to observe the 
TABLE II

Main Types of Energetic Ions in JET Plasmas with a D-T Mixture D:T $=50: 50$, Their Initial and Critical Energies, and Ratio Between Energies Flowing from These Ions to Thermal Ions $G_{i}$ and to Thermal Electrons $G_{e}$

\begin{tabular}{|l|c|c|c|c|}
\hline & $\begin{array}{c}E \\
(\mathrm{keV})\end{array}$ & $E_{c r i t} / T_{e}$ & $\begin{array}{c}E / E_{c r i t} \text { for } \\
T_{e}=14 \mathrm{keV}^{\mathrm{a}}\end{array}$ & $\begin{array}{c}G_{i} / G_{e}= \\
G_{i} /\left(1-G_{i}\right)\end{array}$ \\
\hline Fusion alpha particles & $3.52 \cdot 10^{3}$ & 33 & 7.62 & 0.3 \\
Deuterium NBI & 140 & 16.5 & 0.61 & 5.67 \\
Tritium NBI & 160 & 25 & 0.46 & 9 \\
ICRH-accelerated hydrogen & $\approx 500$ & 8.25 & 4.33 & 0.54 \\
\hline
\end{tabular}

a The estimate of $E / E_{c r i t}$ is given for electron temperature $14 \mathrm{keV}$ achieved in highest fusion power discharge [JET pulse 42976 (Ref. 10)].

effect. The results of the JET experiments demonstrating plasma heating by the alpha particles are reviewed in Sec. II of this paper.

Alfvén instabilities are an important issue for burning plasma studies (see, e.g., Ref. 9 and references therein). Since alpha particles are born in fusion reactions at a speed exceeding the Alfvén velocity, these alpha particles may excite Alfvén eigenmodes via Landau resonance during the alpha-particle slowing down. The spectrum of AEs (frequencies and most unstable mode numbers) and the saturation amplitudes of AEs in burning plasmas constitute a major source of uncertainty, which can significantly affect alpha-particle pressure profiles and alpha-particle losses. AEs are observed in the majority of JET discharges with ICRH (Ref. 17); recent developments in diagnosing the modes via electron density perturbations ${ }^{18}$ significantly expanded the reliability and accuracy of AE detection. Usually, frequencies of the observed AEs in JET are found to be in robust agreement with the existing spectral MHD codes on JET so that obtaining information on plasma parameters from the observed AE spectrum (MHD spectroscopy) becomes possible on JET (Ref. 19). It is important to note, however, that during the high-fusion power D-T experiments, ${ }^{9}$ unstable AEs were not observed in hot ion $\mathrm{H}$-mode even at the highest fusion power. On the other hand, in the optimized shear plasmas aiming at developing internal transport barriers ${ }^{20}$ (ITBs), AEs were driven unstable by the ICRH-accelerated minority ions when ICRH power as low as $1 \mathrm{MW}$ was applied so that possible AE instabilities driven by alpha particles could not be unambiguously identified. ${ }^{9}$ Experimental data on AEs observed in JET and theoretical considerations of the AE stability in JET high-power D-T experiments are presented in Sec. III.

In parallel with the studies of unstable AEs excited by fast ions in JET, stable AEs were studied with external Alfvén antennas. ${ }^{17,21,22}$ In this technique, the relevant Alfvén frequency range is probed with an externally launched wave of sweeping frequency. AEs are detected as high-quality, $\omega / \gamma \cong 10^{2}$ to $10^{3}$, resonances in the plasma response at the AE frequency $\omega$. The damping rate $\gamma$ of such AEs can then be deduced from the width of the resonance. These studies of AEs are presented in Sec. IV.

Diagnosis of energetic ions and energetic ion-driven instabilities is of major importance for burning plasma devices. ${ }^{1}$ This important problem is challenging, too, since the burning plasma diagnostics under the harsh conditions of a D-T plasma must be capable of performing accurate simultaneous measurements of several populations of energetic ions: fusion-born alpha particles, NBIproduced $\mathrm{D}$ ions in the $\mathrm{MeV}$ energy range, and minority ions accelerated with ICRH (Ref. 1). In addition, reliable techniques for detecting electromagnetic plasma oscillations coupled to some of these energetic ions via the wave-particle resonances, such as AEs, are also required since these perturbations may affect transport and losses of the energetic ions. Section $\mathrm{V}$ reviews the development and testing of diagnostic techniques measuring the distribution functions of energetic ions and energetic iondriven instabilities in JET during the decade 1996 to 2006. Several new diagnostics of both confined and lost energetic ions installed in JET in 2006 and 2007 are also described briefly in Sec. VI.

The authorship of this chapter is as follows: Section I (the Introduction) was written by S. E. Sharapov and L.-G. Eriksson. Section II was written by P. R. Thomas and edited by S. E. Sharapov. Section III was written by S. D. Pinches and S. E. Sharapov. Section IV was written by A. Fasoli and D. S. Testa. Section V was written by G. Gorini, J. Kallne, V. G. Kiptily, A. A. Korotkov, A. Murari, and S. E. Sharapov. Section VI (the Conclusions and Outlook) was written by S. E. Sharapov.

\section{OBSERVATION OF ALPHA HEATING IN JET D-T PLASMAS}

The original tokamak reactor concept was based on the idea that once ignited, the D-T plasma in a tokamak 
becomes entirely self-heated through the fusion-born alpha particles, which carry $20 \%$ of the D-T fusion power. ${ }^{1}$ For the alpha-particle power to be absorbed, the alphas must be magnetically confined and slow down in the plasma. Thus, the alpha particle generation, heating, and transport must be understood well enough to predict with certainty alpha-particle behavior in a fusion reactor. A direct experimental investigation of the effectiveness of the alpha-particle heating ${ }^{16}$ was of crucial importance in the D-T experiments performed in JET since only such an investigation could confirm alpha heating models applied to tokamak reactor designs such as ITER.

For fusion plasmas, the energy of fusion-born alpha particles is about an order of magnitude higher than the critical energy at which the power from alpha particles to the electrons equals that to the bulk ions. Thus, the alphaparticle heating of electrons is the dominant effect produced by the alphas during their slowing down, and the electron temperature measurement was considered as the main indicator of alpha heating. For typical electron temperatures of $10 \mathrm{keV}$, more than $95 \%$ of the alpha power is transferred to the electrons.

The TFTR team were the first to observe alpha heating. ${ }^{15}$ The alpha power was $3 \%$ of the total heating power absorbed by the plasma, so the electron heating due to alphas was only twice the error arising from pulse-topulse variation. With a fusion power gain three to four times that of TFTR, JET was in a better position to observe alpha heating. The TFTR experiment established the importance of eliminating isotopic effects, due to the change from D to D-T fuel, and averaging out, or eliminating, the effects of MHD instabilities.

In addition to possible effects due to anomalous plasma thermal conductivity, the isotopic mixture of the plasma and NBI influences heating deposition profiles, electron-ion coupling, neutral penetration, and plasma rotation frequencies, with the latter affecting MHD stability of the plasma. Thus, if alpha heating is to be observed when it is much less than the total power input to the plasma, these isotopic effects must be eliminated. This was accomplished in this experiment by scanning the plasma mix from pure $\mathrm{D}$ to as nearly pure $\mathrm{T}$ as could be managed. Since the alpha production at the extremes of the scan should be small, a maximum must lie in between that should be visible in the plasma temperature.

A 3.8-MA/3.4-T NBI-heated hot-ion $\mathrm{H}-$ mode $^{23}$ was chosen for the alpha heating experiment. It featured a low target density $\left(\sim 1 \times 10^{19} \mathrm{~m}^{-3}\right)$ and low levels of neutral recycling to achieve the hot ion regime. The NBI provided a large core particle source, so its D-T mixture had to be varied in concert with that of the plasma. To maintain constant power and little variation in the particle source, the power was constrained, by features of the JET NBI system and its operation with tritium, to $10.5 \mathrm{MW}$ and the scan to 5 points. Nevertheless, the plasma density, energy content, and fusion yield grew continuously until terminated by a type I edge-localized mode ${ }^{24}$ (ELM),
2.5 to $3 \mathrm{~s}$ after the start of NBI heating. The highperformance phase is significantly longer than the central alpha-particle slowing-down time and the plasma energy confinement time, both of which are $\sim 1 \mathrm{~s}$. The NBI particle source varied from $6 \times 10^{20}$ atoms $/ \mathrm{s}$ in T to $8 \times 10^{20}$ atoms/s in D. The volume average electron density just before the ELM was $\sim 4 \times 10^{19} \mathrm{~m}^{-3}$ for all the pulses in the scan. The TRANSP code ${ }^{25}$ predictions indicated that the fusion output would be 5 to $6 \mathrm{MW}$ with a 50:50 D-T mixture and that the alpha heating should be 30 to $40 \%$ of the power input to the core electrons.

In order that the recycling composition was the same as that of the gas and NBI sources, the vacuum vessel walls and divertor target had to be loaded with the required D-T mixture. This was done by loading up to $\sim 90 \%$ tritium first, using high-density ICRH tritiumfueled pulses followed by the alpha heating pulse type with $3 \mathrm{MW}$ of ICRH and no NBI. The use of ICRH kept the neutron yield to acceptably low levels, and the final use of the alpha heating pulse restored the required low recycling conditions. Subsequent pulses with lower tritium content were prepared in the same way, using deuterium-fueled plasmas to load the walls and the required mix for the ICRH rehearsal of the alpha heating pulse. The first attempt at an $\sim 100 \%$ tritium plasma was somewhat unsatisfactory. Although $\mathrm{T}_{\alpha} / \mathrm{D}_{\alpha}$ light emission, a neutral particle analyzer, and Penning discharge spectroscopy of the divertor exhaust gas indicated that the edge tritium level was $90 \%$, it was clear from the neutron yield that the core concentration was closer to $75 \%$. There must have been a wall source of deuterium that was not seen by the diagnostics. The rest of the scan was completed from this starting point, and the $100 \%$ tritium pulse was repeated after an intensive period of pure tritium fueling for other purposes. This time, the neutron yield was about $15 \%$ of that obtained with $60 \%$ tritium and corresponded to a core tritium concentration of $92 \%$.

The fusion performance of these plasmas turned out to be slightly better than anticipated. The best was pulse 42847, which produced a maximum of 6.7-MW fusion power with $60 \%$ tritium concentration. Some traces for this pulse are shown in Fig. 2, together with those for pulse 43011 , with $92 \%$ tritium. The alpha heating power is computed in TRANSP using the computed birth profile normalized to the measured fusion rate. The trapping and slowing down of the alphas are modeled in a Monte Carlo package, similar to that for NBI. It can be seen that although the absorbed NBI powers are nearly identical, the $92 \%$ tritium pulse has a very much lower alpha heating power. Since the pure deuterium pulses have essentially zero fusion power, the aim of obtaining a clear maximum in the fusion yield had been achieved. Notice that the neutral beam power of 43011 is larger than that of 42847 for the first $0.5 \mathrm{~s}$. As a result, both energy content and temperatures grow more rapidly at the start. Once it has built up, the effect of the alpha heating in 


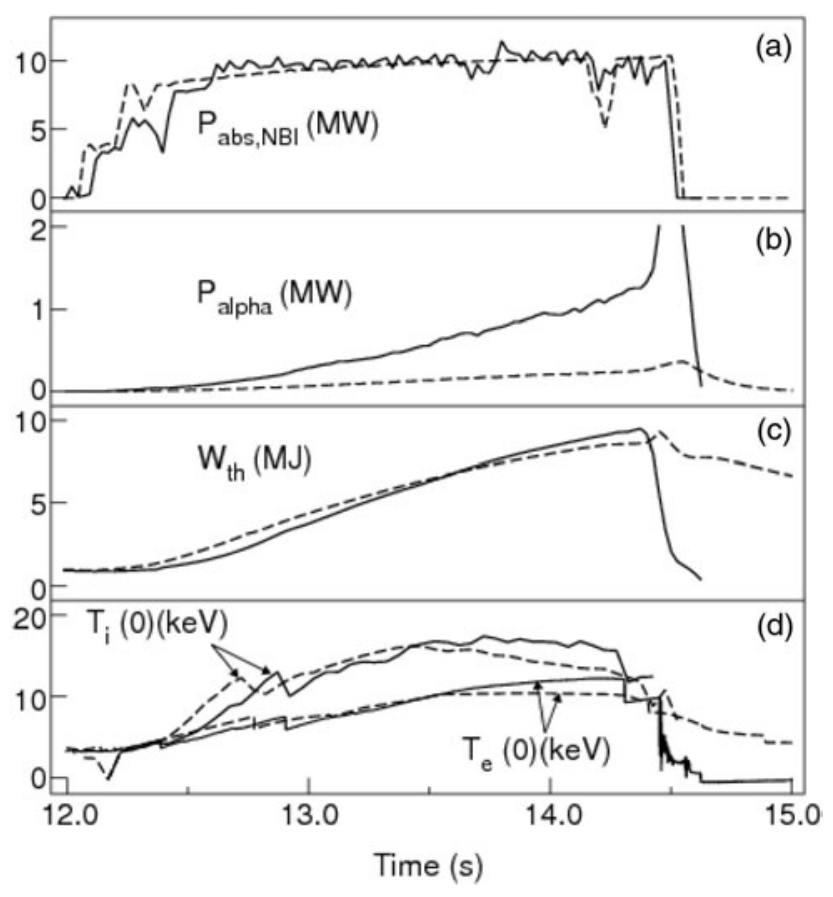

Fig. 2. Traces of (a) absorbed NBI power, (b) alpha heating power, (c) thermal plasma energy content, and (d) central ion (CXS) and electron (ECE) temperatures for pulse 42847 (solid line), which had the largest fusion output of the scan, and pulse 43011 (dashed line), which had the largest tritium content. ${ }^{16}$

42847 is apparent during the last second of the beam pulse. The ion temperature increase is due to changes in equipartition and NBI power to the electrons rather than direct alpha heating.

\section{II.A. Alpha-Particle Heating of Electrons}

The alpha heating of the electrons can be seen clearly in the contour plot of the central electron temperature, determined from electron cyclotron emission (ECE), versus time and D-T mixture, which is shown in Fig. 3. The $\mathrm{D}-\mathrm{T}$ mixture is adjusted to reconcile TRANSP estimates of the peak neutron yield, using experimental temperature and density profiles, with the experimental values. The maximum $T_{e}$ grows with increasing tritium concentration, up to a maximum at $60 \%$, and then declines to nearly the same value as in pure deuterium. The effect of sawteeth is strongest at low $\mathrm{T}$ concentration, and alpha heating is best seen in the electron temperature reached just before sawtooth crashes. This is why pulses 42847 and 43011 were compared in Fig. 2.

The error in the value of the neutron-based D-T mixture is $3 \%$ at 10 or $90 \%$ T, increasing to $20 \%$ at 40 or $60 \%$ $\mathrm{T}$ because the yield is insensitive to the mixture in this range. Part of this error is due to an uncertainty in $Z_{\text {eff }}$ that has been corrected once the optical transmission of

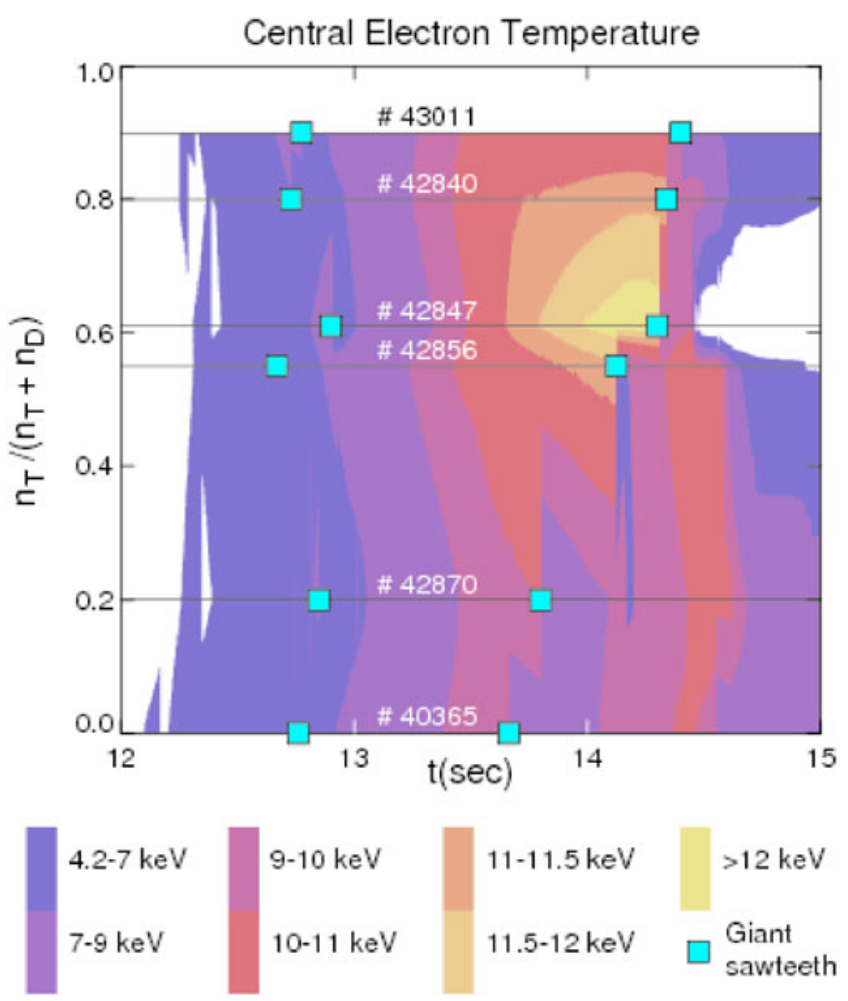

Fig. 3. Contour plot of the central electron temperature against time (horizontal axis) and $n_{\mathrm{T}} /\left(n_{\mathrm{T}}+n_{\mathrm{D}}\right)$ (vertical axis) for the six pulses of the alpha heating scan. Giant sawteeth are marked with squares. ${ }^{16}$

vacuum vessel windows has been measured. At the time, the peak yield was estimated to be closer to $50 \%$.

The late phase of the $20 \%$ tritium pulse and some other pulses, whose data have not been included, showed a clear deterioration of the central electron temperature when edge MHD activity was present. In the following, data were selected from the peak of sawteeth when such activity was not present.

Using data selected as described above, the alpha heating can be brought out very clearly. Figure 4 shows the central electron temperature against total heating power, including alpha heating, determined by TRANSP. The horizontal bars indicate the difference in absorbed NBI power compared with the $92 \%$ tritium pulse, 43011. Bars to the right indicate a relative shortfall in NBI power. It can be seen that this difference is as much as $1 \mathrm{MW}$ for some of the points at low tritium concentration. However, most of the alpha scan points are within $0.3 \mathrm{MW}$ of NBI power in 43011. The figure includes for comparison some ICRH pulses, which had been used before the tritium experiment to test the feasibility of detecting alpha heating. The effect of ICRH is very similar to that of alpha heating because it couples mainly to the plasma electrons, as discussed in the Introduction. A number of points about Fig. 4 are apparent: 


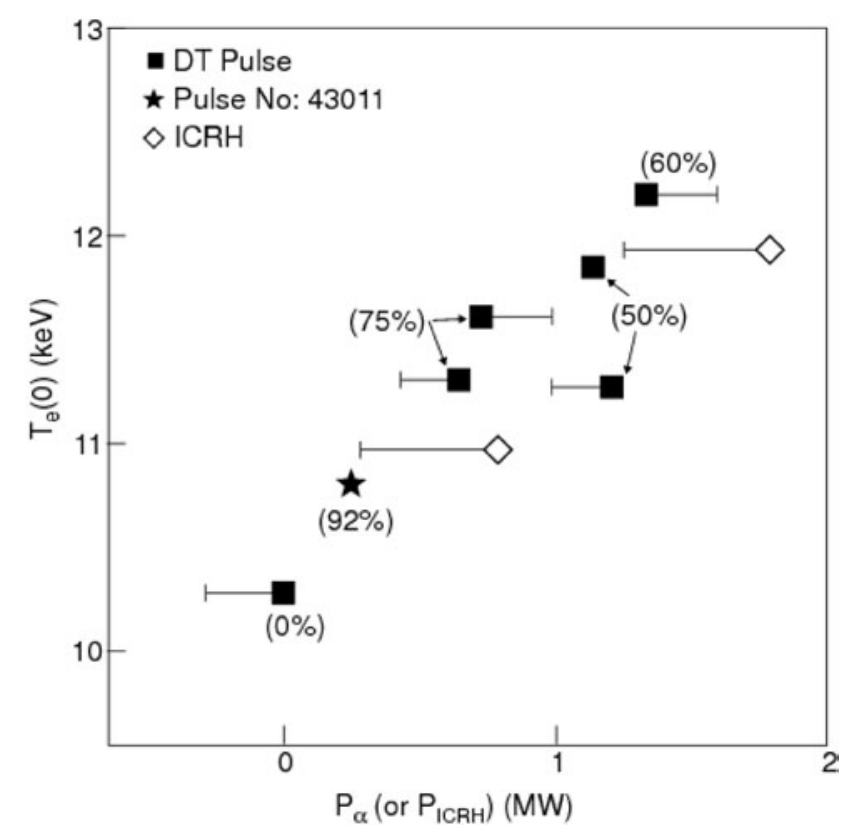

Fig. 4. Central electron temperature (ECE) versus total heating power, which includes alpha-particle heating. ${ }^{16}$ The D-T data (squares and star) and the ICRH emulation data (diamonds) are identified. The bars indicate the variation in NBI power compared to the $92 \% \mathrm{~T}$ pulse 43011.

1. The temperature is proportional to the total heating power.

2. The bars show that the correlation between the points improves if the actual NBI power is used.

3. The alpha and ICRH power sources are identical in their effects.

A regression fit to the data of Fig. 4 gives $T_{e}(0)=$ $(0.21 \pm 0.99)+(0.99 \pm 0.09) P_{\text {heat }}$, where $P_{\text {heat }}$ is the total power absorbed by the plasma, including ICRH and alpha power. Separating the alpha power in the fit gives $T_{e}(0)=(0.07 \pm 1.04)+(1.0 \pm 0.1)\left(P_{\text {heat }}-P_{\alpha}\right)+(0.99 \pm$ 0.13) $P_{\alpha}$. If the electron density or $n_{\mathrm{T}} / n_{\mathrm{D}}$ is included in the regression fit, their weights are zero within errors. The lack of any significant mixture dependence indicates that there can be no isotopic effect in energy confinement. The lack of dependence of electron density with $T_{e}$ is consistent with the simultaneous increase in plasma energy content and density during the ELM-free phase of these plasmas. The regression fit gives a change in central $T_{e}$ of $1.3 \pm 0.23 \mathrm{keV}$ with $1.3-\mathrm{MW}$ alpha power. The standard error for the temperature is indicated, which reflects the pulse-to-pulse variation in the data. It should be remembered that the data are selected to be free of MHD activity and that there are systematic calibration errors for $T_{e}(0)$ and $P_{\alpha}$, which are estimated to be 5 and $10 \%$, respectively.
The regression analysis of the $T_{e}$ data showed that there was no discernible isotope effect in the electron heating. This is confirmed by the dependence of the thermal energy confinement time on mixture. Figure 5 shows the diamagnetic and thermal energies together with the plasma energy confinement time versus D-T mixture. The thermal energy content and energy confinement time are those obtained from the TRANSP analysis. The error bars in the energy confinement time reflect the fluctuations in value from one time slice to the next and are not statistically based. The data points are taken when the diamagnetic energy content peaks, which is normally just before the end of the ELM-free phase. The difference between the energy contents mainly reflects the energy contained in the slowing-down NBI ions; this can be as much as 2 MJ. The maximum alpha energy content is 0.25 MJ. The alpha heating is visible in the thermal energy. The figure shows that the thermal energy confinement time is the same for pure $\mathrm{D}$ and pure $\mathrm{T}$ plasmas, within errors. Thus, if there is any isotopic dependence in the plasma thermal conductivity, it is rather weak. The increase in errors for the pulses with significant alpha heating is due to the larger $d W / d t$ term. That there is a slight increase in confinement time as well is due to the more favorable alpha-particle deposition profile and to the resulting peaking of the pressure profile.

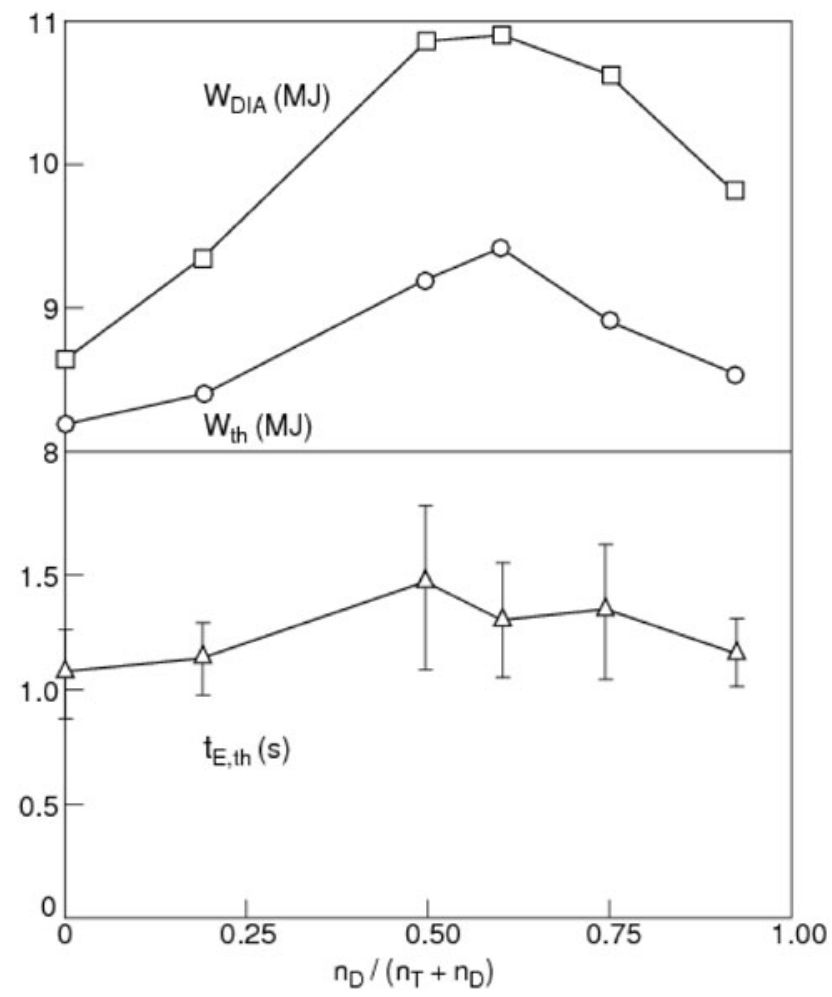

Fig. 5. Diamagnetic and thermal energy contents (top) with the plasma energy confinement time versus D-T mixture (bottom). ${ }^{16}$ 


\section{II.B. Alpha-Particle Heating of Bulk lons}

It was apparent in an early study of the data that the ion heating was larger than expected from the reference deuterium discharges and that analysis required a reduction of the ion thermal conductivity in the presence of alphas in D-T plasmas. ${ }^{26}$ The charge-exchange (CXS) data have subsequently been thoroughly checked and the latest corrections incorporated. The unexpected ion heating is still valid. It is important to note that the published results on electron heating are unchanged by subsequent analysis. Figure 6 shows that the increase in $T_{i}$ was larger than that of $T_{e}$ even though the alpha power to the ions was around $15 \%$ of the total alpha power. The ion temperature is very similar in the 0 and $92 \%$ tritium plasmas.

The independent measurements of $W_{\text {dia }}$ and of the neutron rates are in good accord with the TRANSP output, which uses $T_{i}(r)$ obtained from the CXS measurements. The efficiency of the ion heating with alphas in $\mathrm{D}-\mathrm{T}$ plasmas is three times that of the electrons, and this ion heating is not observed with ICRH substituting for alphas in deuterium discharges, although these plasmas show the same level of electron heating. The classical alpha heating of ions should be negligible. Also, the equipartition power, in the confinement region, hardly changes across the mixture scan. In contrast to the pulses with alpha heating, the ICRH pulses show ion temperature rises comparable to those of the electrons, as expected due to equipartition. It is hardly possible that either the

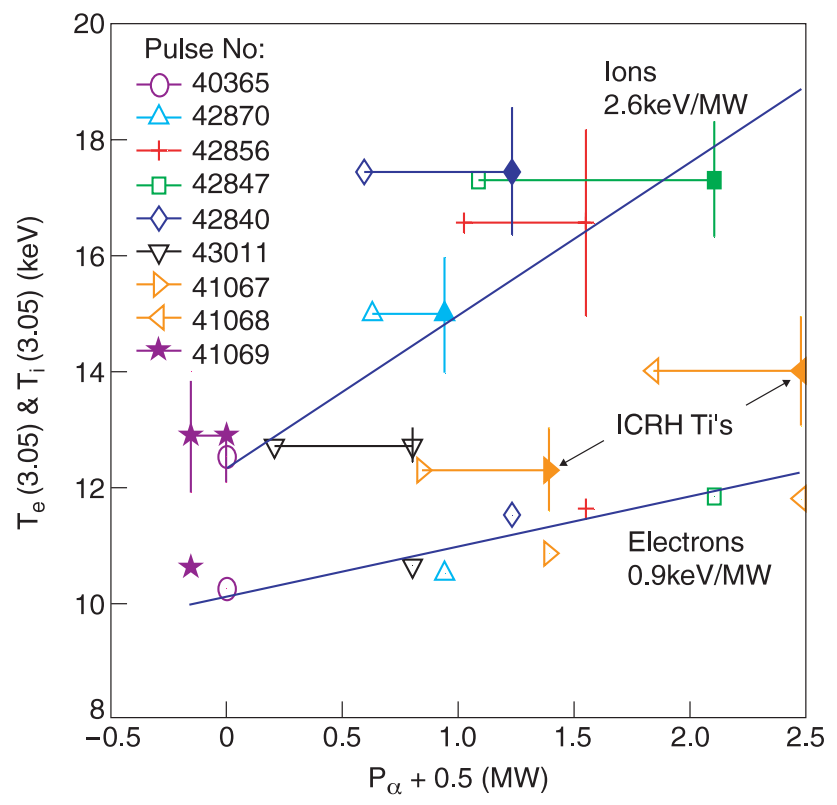

Fig. 6. The central ion and central electron temperatures for the D-T mixture scan and the ICRH emulation series. The bars indicate the change in NBI power relative to the $0 \%$ T pulse 40365 (note the difference with Fig. 4). The fitted lines are to D-T data only. ${ }^{26}$
CXS $T_{i}$ or the NBI power measurements are at fault due to the following reasons:

1. All the data validation using CXS $T_{i}$ hold up very well. The magnetic energy contents and $14-\mathrm{MeV}$ neutron yields are reproduced well by the profile data.

2. Some of the NBI power variation, across the scan, is of the same order as the alpha heating power. Goodquality fits to the electron temperature data result, if this variation is included. Thus, the NBI power measurements seem to be consistent with the ECE data.

The $T_{i}$ profiles shown in Fig. 7 give an impression of barrier formation with a marked increase in $d T_{i} / d r$. The ion thermal conductivity decreases, but to nothing like the neoclassical value. However, no evidence of an alphaspecific mechanism for ITB formation has been found for these discharges. Toroidal Alfvén eigenmodes ${ }^{27}$ (TAEs) and elliptical Alfvén eigenmodes ${ }^{28}$ (EAEs) were not detected with external magnetic pickup coils in these discharges. It is possible, however, that compressional Alfvén eigenmodes ${ }^{29}$ were present at much higher frequency, as proposed for anomalous ion heating in NSTX. Ion cyclotron emission monitors were not available during this experiment.

\section{II.C. Summary}

Alpha-particle heating has been unambiguously observed in JET D-T plasmas. A scan of D-T mixture was used successfully to separate the effects of alpha heating and potential isotopic dependence of energy confinement. A change in central electron temperature of $1.3 \pm$ $0.23 \mathrm{keV}$ is ascribed to $1.3 \mathrm{MW}$ of alpha heating. The

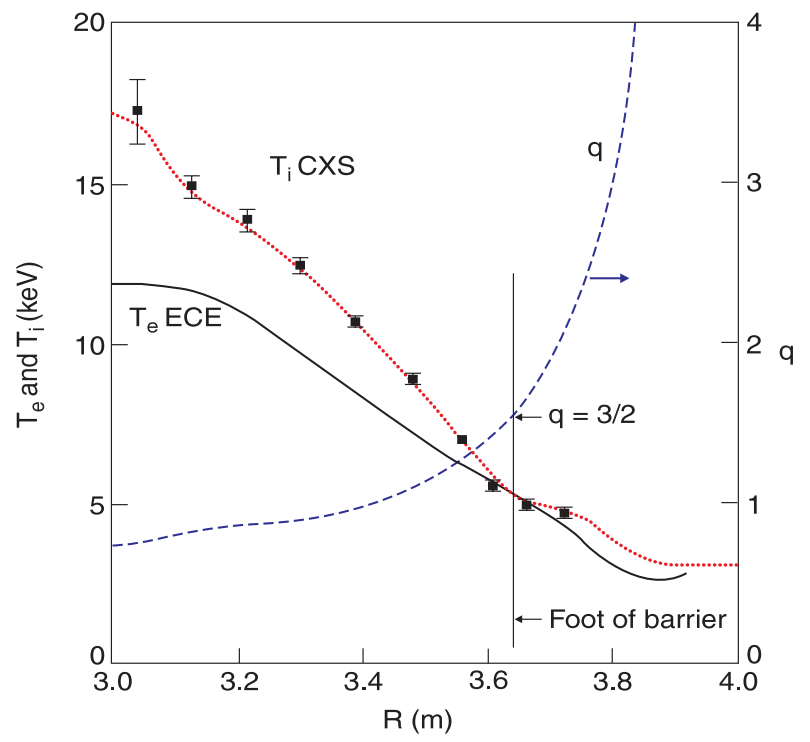

Fig. 7. Possible evidence for ion transport barrier at $q=3 / 2$ magnetic surface. ${ }^{26}$ 
scan showed that the plasma energy confinement in the hot-ion $\mathrm{H}$-mode regime has no significant isotopic dependence. With a plasma energy confinement time of $1.2 \mathrm{~s}$, the alpha heating produced an increase of plasma energy content of more than $1 \mathrm{MJ}$ in $9 \mathrm{MJ}$. Alpha heating was observed, in this study, to be as effective as hydrogen minority ICRH. This is a strong indication that there are no unpleasant surprises with respect to alpha heating of electrons and that there are no anomalous effects on trapping or slowing down. Furthermore, it is highly encouraging that the peaked alpha heating profile shows up in the heating rate and the energy confinement time.

The ion heating was found to exceed the classical estimates. Although the origin of this favourable effect is not established yet, it seems that an ion transport barrier observed experimentally might be responsible for this effect. A dedicated study of ion heating may be of great interest for possible high power D-T experiments in JET in future campaigns.

\section{ENERGETIC PARTICLE-DRIVEN AES IN JET D-T PLASMAS}

The sustainment of controlled nuclear fusion requires that the fast charged fusion products are confined for a sufficient time so as to heat plasma, enabling thermal ions to fuse and thus achieve a self-sustained burn. It is additionally required that these energetic fusion ions do not adversely affect the stability of the plasma, leading to a quench of the fusion process. How the plasma stability is affected by the presence of highly energetic populations of ions, in particular $3.5-\mathrm{MeV}$ alpha particles, is a focus of this section. In burning plasma, the fusion-born alpha particles will significantly contribute to the total plasma pressure. Of principal concern is that the alpha-particle population may induce instabilities that cause a radial alpha-particle transport and loss, as could happen due to the excitation of TAEs (Refs. 27 and 30). This is an instability that taps the universal instability drive to cause a radial displacement of the alpha particles. Typically, the universal instability drive is considered a low-frequency mechanism that causes the destabilization of drift waves (variants of which are responsible for the anomalous diffusion of the background plasma). However, because the alpha-particle energy at birth is $\sim 200$ times the thermal ion energy and the drift frequency of a species is proportional to their energy, this mechanism can cause the destabilization of relatively high-frequency waves, in particular TAE modes. Indeed, alpha particles in tokamak plasmas typically have speeds that are somewhat greater than the Alfvén velocity, and thus the resonant coupling of alpha particles to Alfvén waves is quite well matched in future burning plasma experiments.

A fusion reactor can tolerate fast particle losses of only a few percent, primarily due to the resulting first- wall damage that they would cause. It is therefore essential that any burning plasma experiment has a window of operation in which either the TAE modes are stable or, if unstable, the effect of instability leads only to a mild rearrangement of the alpha-particle distribution, rather than direct loss of alpha particles to the surrounding walls. A mild redistribution of the alpha particles still allows for the self-heating of the plasma by alpha particles. However, direct loss of energetic alpha particles reduces the self-heating in addition to causing wall damage. If a "sweet spot" with significant self-heating and alpha-particle production is established, then optimization experiments can be performed to determine the range of plasma parameters that are compatible with ignited operation.

In this section, the stability of alpha-particle-driven AEs is analyzed in high-fusion power D-T discharges in JET. Both hot-ion H-mode ${ }^{10}$ and optimized shear ${ }^{20}$ discharges are considered. Unstable AEs are not observed ${ }^{9}$ in hot-ion H-mode D-T discharges even at the highest fusion power with alpha-particle beta $\beta_{\alpha}(0) \approx 0.7 \%$. A theoretical analysis shows that the $\mathrm{AE}$ stabilization is caused by the large plasma pressure, which prevents the existence of core-localized AEs at peak fusion performance. Kinetic toroidal Alfvén eigenmodes ${ }^{31,32}$ (KTAEs) that persist even at high plasma pressure are found to be radially extended modes and are subject to strong damping. The stability analysis of these modes is based on calculations performed with the CASTOR-K code $^{33}$ that confirms that AEs cannot be driven unstable by alpha particles in high-performance hot-ion H-mode discharges performed in JET. AEs in optimized shear regimes are found to be more unstable than those in the hot-ion $\mathrm{H}$-mode regime, primarily as a result of the elevated central safety factor $q(0)$, which increases the efficiency of the interaction of the AE with the energetic ions. As a consequence, AEs are still observed in optimized shear D-T discharges when ICRH is applied at power levels as low as $1 \mathrm{MW}$, and this effect prevents an unambiguous investigation of AEs driven by fusion-born alpha particles alone. To avoid the uncertainty associated with the AE drive by ICRH-heated energetic ions, we consider a D-T discharge without ICRH (pulse 42677). Although the somewhat higher alpha-particle parameters shown in Table I have been achieved in hot-ion H-modes with ICRH, discharge 42677 with NBI only also had a significant fusion power, $11.5 \mathrm{MW}$.

\section{III.A. Instability Mechanisms and Linear AEs}

It is well known that confined plasmas of species $j$ have a flow, generally called the diamagnetic drift flow,

$$
\mathbf{v}_{* j} \approx \frac{1}{e_{j} B_{j}} \frac{\mathbf{b} \times \nabla\left(n_{j} E_{j}\right)}{n_{j}},
$$

where $n_{j}$ is the density of species $j, q_{j}$ its charge, and $E_{j}$ its mean energy. Further, if $0<\omega<\left\langle\mathbf{k} \cdot \mathbf{v}_{* i}\right\rangle$, (where $k$ is the 
mode number and the angle brackets denote spatial averaging), particle resonance gives rise to an inverse dissipative contribution that can destabilize a wave (the so-called universal instability drive). In thermal plasmas this mechanism is known to contribute to anomalous diffusion where it acts at relatively low frequency. However, because $\omega_{\alpha} \propto\left\langle E_{\alpha}\right\rangle \sim 200 T(\mathrm{keV})$, energetic particles can destabilize waves at relatively high frequencies, in particular shear Alfvén waves. In ideal MHD the shear Alfvén dispersion relation is $\omega^{2}=k_{\|}^{2} v_{A}^{2}$, where $v_{A}=$ $\sqrt{B_{0}^{2} / \mu_{0} \rho_{0}}$ is the Alfvén speed. Shear Alfvén waves represent a balance between plasma inertia and field line tension and are analogous to waves on a taut string.

In inhomogeneous plasmas, such as those found in all magnetically confined fusion-grade devices in which the density $n$, and thus the Alfvén speed, depend upon the minor radius $r$, the shear Alfvén dispersion relation becomes $\omega^{2}=k_{\|}^{2}(r) v_{A}^{2}(r)$. It is clear that no wave packet of finite radial extent can persist for long since each radial "slice" along the wave packet moves with a different velocity in a different direction. Under these conditions, a wave packet excited by resonance with energetic particles can hardly persist for a long time. However, the discovery of weakly dampeds TAE (Ref. 27) in toroidal geometry has changed the view on Alfvén instabilities driven by energetic particles.

In a torus it is natural to represent perturbations of the plasma by Fourier decomposition into poloidal $m$ and toroidal $n$ harmonics,

$$
\xi(r, \theta, \phi)=\sum_{m, n} \xi_{m, n}(r) \exp [i(n \phi-m \theta-\omega t)]
$$

Since the equilibrium magnetic field $B \approx B_{0} R_{0} / R \approx$ $B_{0}\left(1-r / R_{0} \cos \theta\right)$ is a function of $\theta$, a coupling arises between the neighboring poloidal harmonics, and to lowest order in the inverse aspect ratio, $\varepsilon=r / R_{0} \ll 1$, each poloidal harmonic $m$ couples to its nearest neighbor sidebands, $m \pm 1$. The parallel wave number is approximately given by $k_{\| m}=1 / R[n-m / q(r)]$, where $q$ is the so-called safety factor (the inverse of the field helicity $\iota$ ) and can be approximated as $q(r)=r B_{t} / R B_{p}$, where $r$ is the minor radius, $R$ is the major radius, and $B_{p}$ and $B_{t}$ are the poloidal and toroidal magnetic field components, respectively. In this case, the system is described by a set of coupled differential equations for the radial plasma displacement. ${ }^{27,30}$

There is, however, a special degeneracy in a cylinder in which the various branches of the Alfvén continuum cross each other (in Fig. 8a only two branches are shown). This occurs when $\omega=-k_{\| m+1}(r) v_{A}(r)=+k_{\| m}(r) v_{A}(r)$, which is satisfied at $q(r)=(2 m+1) / 2 n$. Near such points in a torus, toroidal coupling resolves the degeneracy by developing a band gap in frequency where phase mixing does not occur.

Within the frequency gap, eigenmode solutions known as TAEs have been found to exist. Since these modes lie within this frequency gap in the Alfvén continuum, they do not experience continuum damping and are therefore damped by weaker dissipative processes. These modes are spatially localized around the extremum of $\omega^{2}$ and typically consist of the two main poloidal harmonics that are associated with the gap. A typical example is shown in Fig. 8b. Besides TAEs, additional coupling mechanisms due to the shaping of the plasma's poloidal cross

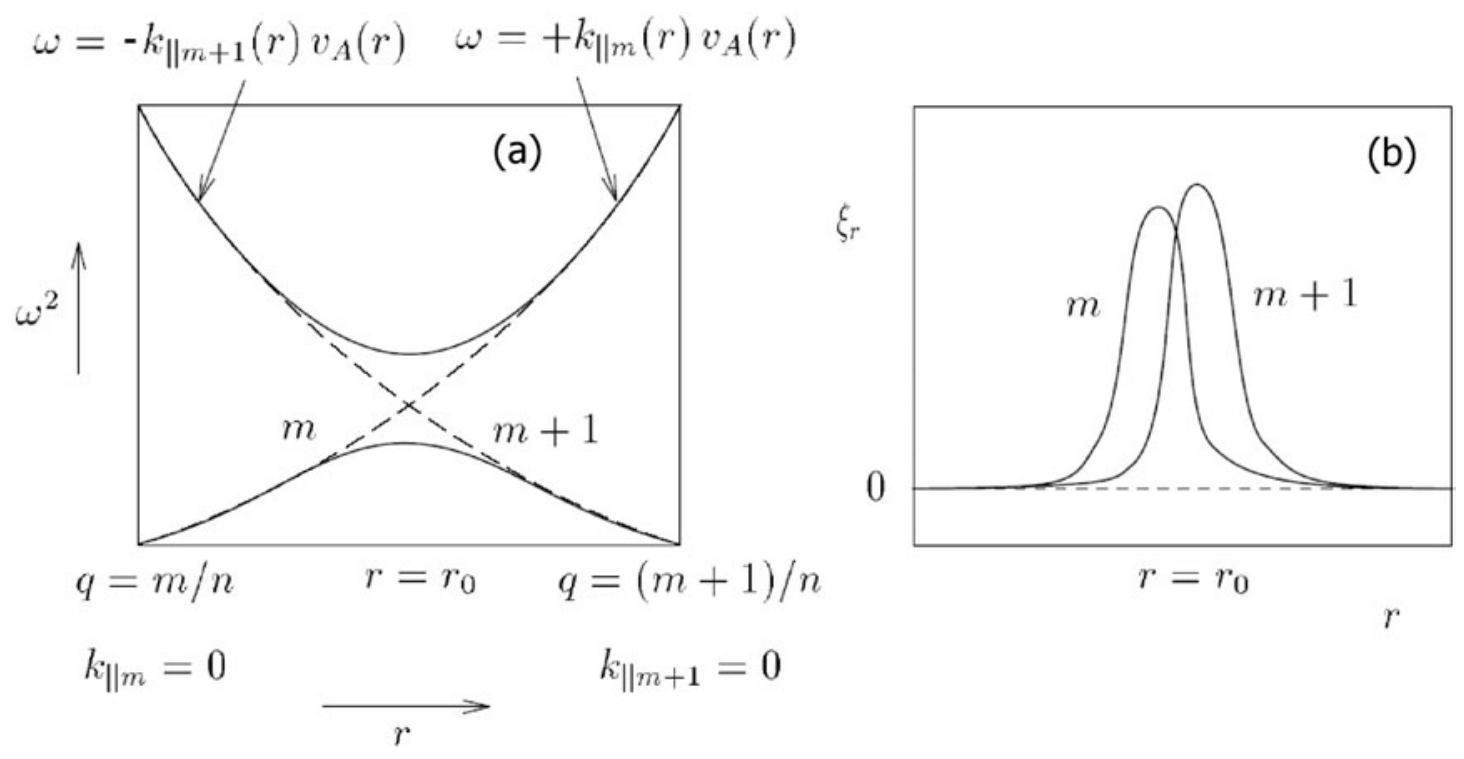

Fig. 8. (a) Alfvén frequency continuum in a torus, showing coupled poloidal harmonics, and (b) the corresponding TAE eigenfunction. 
section allow other gaps to appear in the Alfvén frequency continuum, which support other eigenmodes such as the EAE and the noncircular Alfvén eigenmode (NAE).

\section{III.B. Stability Diagram for Alpha-Particle-Driven AEs in Hot-Ion H-Mode}

The evolution of plasma parameters for an NBI-only D-T discharge with high fusion power is shown in Fig. 9. The MHD analysis of the AE spectrum in D-T discharge 42677 with NBI only, $P_{\mathrm{NBI}}=21.3 \mathrm{MW}$, was performed in the following way: The plasma equilibria are reconstructed by EFIT (Ref. 34) and HELENA (Ref. 35) for different time slices ranging from $t=12.1 \mathrm{~s}$ to $t=13.4 \mathrm{~s}$. A typical equilibrium and the corresponding structure of the Alfven continuum are shown in Fig. 10. TAEs and core-localized TAEs (Ref. 36) exist for these equilibria only at early times, i.e., for $t<13.0 \mathrm{~s}$. No ideal MHD TAEs or core-localized TAEs were found in the region $r / a \leq 0.5$ at later times. The absence of any ideal MHD TAE and core-localized TAE is caused by the pressure effect. ${ }^{9}$

The only AEs that are found in the plasma center at the time of peak performance, $t>13.0 \mathrm{~s}$, are the KTAEs (Refs. 31 and 32). These modes are computed by the

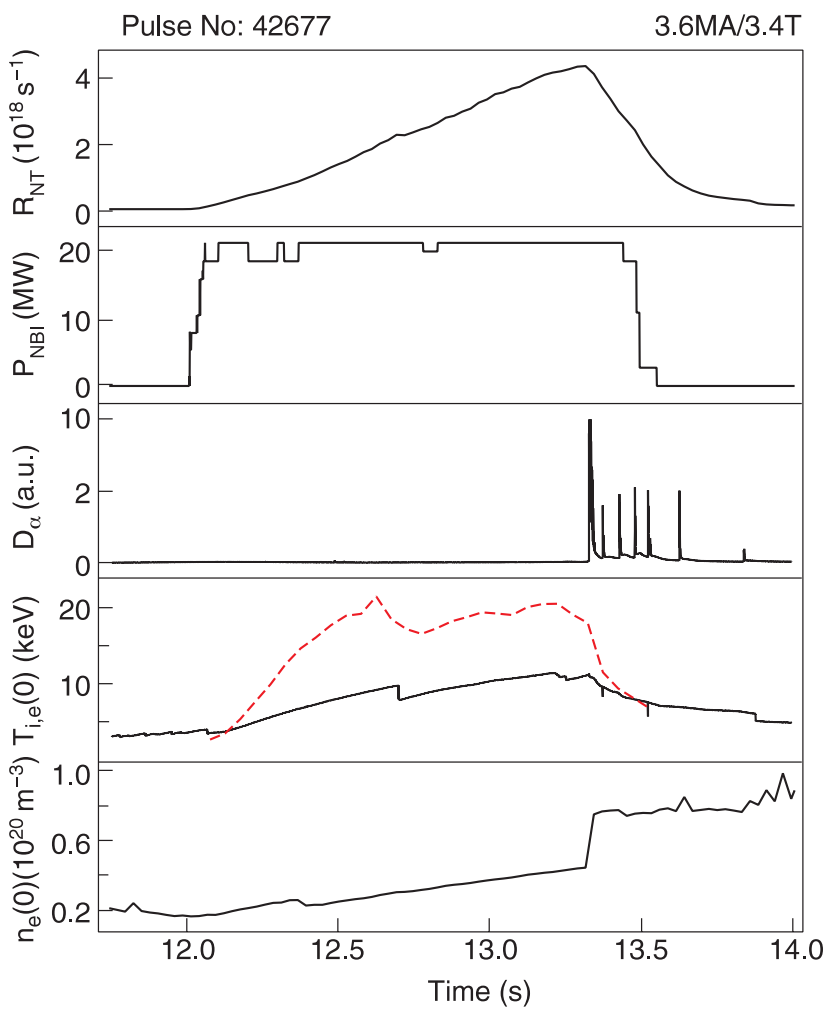

Fig. 9. Temporal evolution of the D-T fusion rate, NBI power, $D_{\alpha}$ signal, central ion (broken line) and electron (solid line) temperatures, and central electron density in D-T discharge 42677 in JET (Ref. 9).
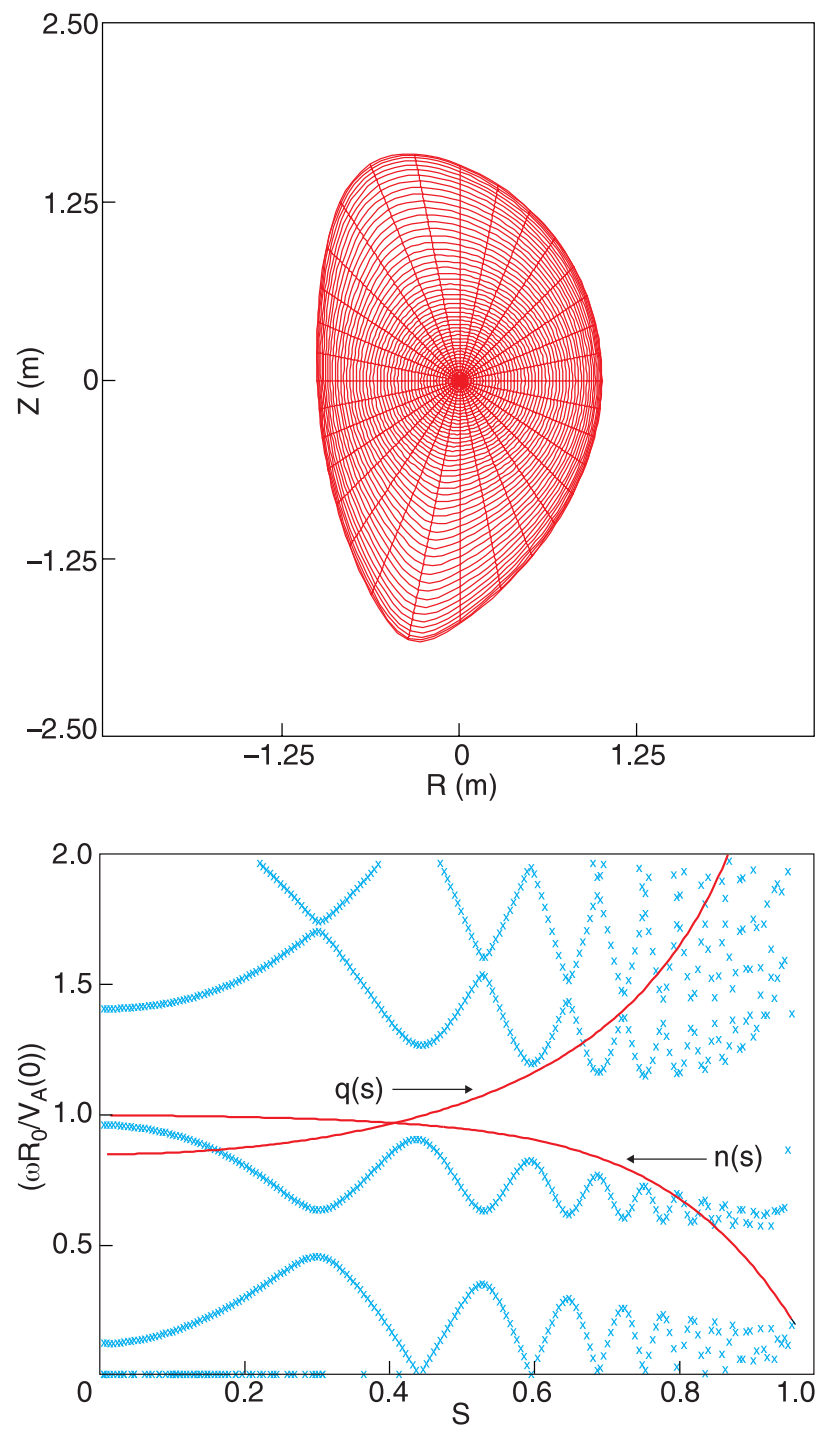

Fig. 10. The reconstructed equilibrium ${ }^{9}$ for D-T discharge 42677 at $t=13.3 \mathrm{~s}$, together with the Alfvén continuum structure. $q(s)$ and $n(s)$ are the safety factor and electron density radial profiles; $q(0)=0.85$.

CASTOR code. ${ }^{32}$ The maximum values of the growth rate are reached by AEs with toroidal mode numbers $n \approx r_{0} / \Delta_{\alpha}\left(r_{0}\right.$ is the mode localization radius and $\Delta_{\alpha} \approx$ $q \rho_{\alpha}$ is the alpha-particle drift orbit width ${ }^{32}$ ). Thus, KTAEs with intermediate mode numbers, $n=4$ to 8 , are of major interest since they have the most effective drive. These normal modes constitute the input for the stability analysis. The radial structure of the most unstable KTAE with toroidal mode number $n=6$, computed for the time slice $t=13.3$, is shown in Fig. 11. One can see that this KTAE consists of many coupled poloidal harmonics and is therefore a radially extended mode; varying $q(0)$ within $15 \%$ [keeping the profile $q(r)$ fixed] does not affect significantly the radial extent of the mode. 


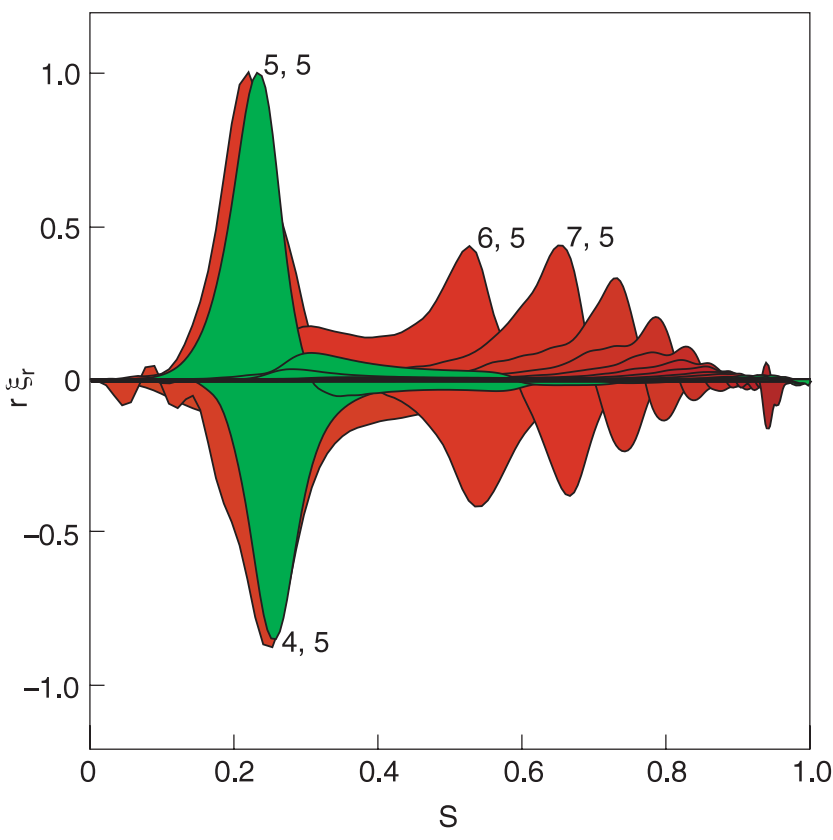

Fig. 11. The computed nonideal KTAE (red color online or dark gray in black and white) with eigenvalue in D-T discharge 42677 at $t=13.3 \mathrm{~s}$. In addition, the most unstable upper core-localized TAE analyzed in the modeling prior to the D-T experiments is shown (green color online or gray in black and white). ${ }^{9}$

Analysis has been performed on whether the $n=6$ mode shown in Fig. 11 can be linearly unstable in the presence of the alpha-particle drive. The drive competes with the bulk ion and the beam ion Landau damping, the radiative damping, and the electron collisional and Landau damping. The alpha-particle contribution and Landau damping are computed by the complex resistivity CASTOR-K code $^{33}$ with finite drift orbit and global mode structure effects taken into account. It is found that the alpha-particle drive at the time of the peak performance yields a normalized growth rate $\gamma_{\alpha} / \omega=0.27 \%$, while the bulk deuterium Landau damping is $\gamma_{\mathrm{D}} / \omega=-0.45 \%$, the bulk tritium Landau damping is $\gamma_{\mathrm{T}} / \omega=-0.2 \%$, and the beam Landau damping is $\gamma_{b} / \omega=-0.53 \%$. The radiative and electron collisional damping rates are computed by CASTOR, giving $\left(\gamma_{R}+\gamma_{e}\right) / \omega=-0.23 \%$.

Adding all the damping effects, it is evident that the KTAE is completely stable under these conditions. The radial extent of the eigenfunction causes the bulk plasma damping to stabilize the mode across almost the entire minor radius, whereas the alpha-particle drive is effective only at the very central part of the KTAE. To demonstrate the importance of the radial width of the eigenfunction, one can compare the computed $n=6 \mathrm{KTAE}$ with one of the upper core-localized TAEs used in the predictive modeling of the most unstable scenarios ${ }^{33}$ (see Fig. 11). It is seen in Fig. 11 that in contrast to the KTAE, the upper core-localized TAE is localized in the central region, where the alpha-particle distribution is localized. The CASTOR-K analysis shows that this mode (provided it exists in D-T discharges) could become marginally unstable at peak plasma performance. This conclusion can be best illustrated on the AE stability diagram (see Fig. 12), in which the instability regions for both alphaparticle-driven KTAEs and core-localized TAEs from Fig. 11 are shown as functions of the alpha-particle pressure and the plasma density (in the form of the parameter $\left.V_{\alpha} / V_{A}\right)$. The evolution of the alpha-particle pressure and of the plasma density during D-T discharge 42677 is computed by the TRANSP code. It is seen from Fig. 12 that the KTAE instability domain could not be reached during the actual D-T discharge, whereas the upper corelocalized TAE (provided it exists) could become marginally unstable at peak plasma performance.

\section{III.C. AEs in High-Fusion Power-Optimized Shear D-T Plasmas in JET}

The optimized shear scenarios ${ }^{20}$ generate very promising regimes of enhanced plasma confinement at a reduced value of inductive current of tokamak. The JET record in D-D fusion performance was achieved in an

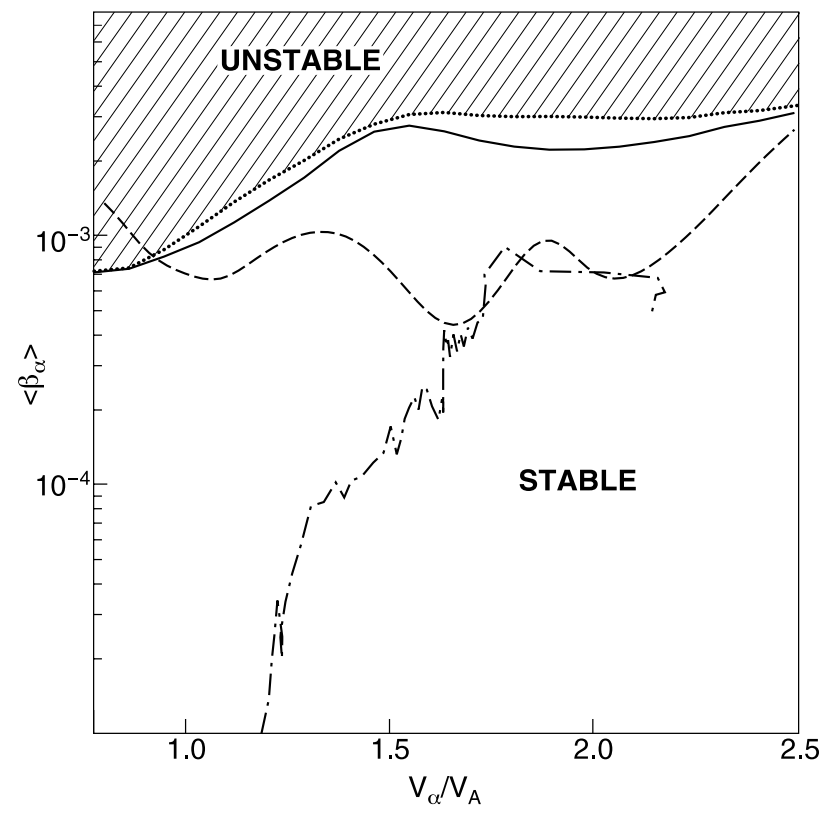

Fig. 12. The instability zone computed by the CASTOR-K code for alpha-particle-driven KTAEs shown in Fig. 11. The instability boundary without the stabilizing effect of the beam is shown by the solid line. The instability boundary for the upper core-localized TAE from Fig. 11 is also shown for comparison (dashed curve). The actual path of the parameters in discharge 42677 is computed by the TRANSP code and is marked by the dotted-dashed curve. ${ }^{9}$ 


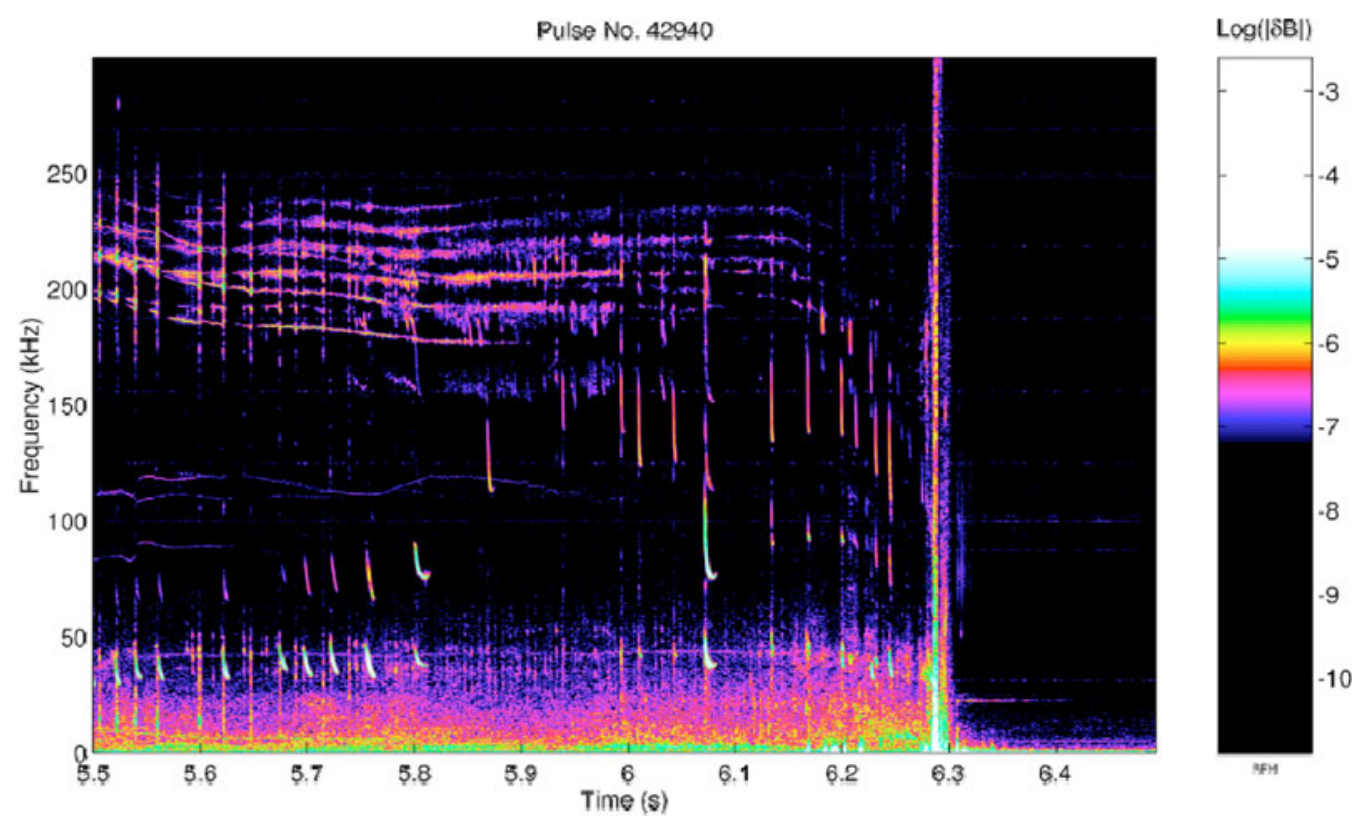

Fig. 13. Spectrogram ${ }^{9}$ of the magnetic perturbations measured by the external Mirnov coils in shear-optimized D-T discharge 42940 with high fusion power. Multiple AEs with different toroidal mode numbers ranging from $n=4$ to $n=7$ are observed at frequencies $f_{n}^{L A B}=180$ to $250 \mathrm{kHz}$. A disruption occurs at $t=6.3 \mathrm{~s}$.

optimized shear discharge; i.e., the rate of D-D fusion was $R_{\mathrm{DD}}=2 S_{n} \approx 1.1 \times 10^{17} \mathrm{~s}^{-1}$ in discharge 40554. In contrast to the hot-ion H-mode, where the transport barrier appears at the plasma edge, the optimized shear regimes are characterized by an ITB. Due to the high value of the safety factor, $q(0)>1$, and the absence of highpressure gradients near the separatrix, the optimized shear regime benefits from the absence of sawteeth and ELMs, which are the most destructive MHD phenomena in H-mode plasmas.

To set up such an ITB, a significant ICRH power is applied in JET, i.e., $P_{\mathrm{ICRF}} \geq 6 \mathrm{MW}$. The corresponding ICRH-accelerated energetic ions drive AEs at high safety factor and low initial plasma density unstable at all times during the discharge. In the absence of sawteeth and ELMs, the AEs and so-called "chirping modes" constitute the dominant MHD phenomena, which are observed on the magnetic pickup coils, as Fig. 13 shows. It is emphasized that no obvious deterioration of the plasma performance due to AEs was observed in JET. The evolution of the plasma parameters for discharge with modes shown in Fig. 13 is displayed in Fig. 14. Several electromagnetic modes with toroidal mode numbers ranging from $n=4$ to $n=7$ are observed during the entire discharge up to the time of the disruption, at $t=6.3 \mathrm{~s}$. The mode frequencies in the laboratory reference frame at $t=5.5 \mathrm{~s}$ are 200 $(n=4), 214(n=5), 226(n=6)$, and $249(n=7) \mathrm{kHz}$. The estimate of the mode frequencies in the plasma reference frame with the Doppler effect due to toroidal plasma rotation, $f_{\text {rot }} \approx 13 \mathrm{kHz}$, taken into account gives

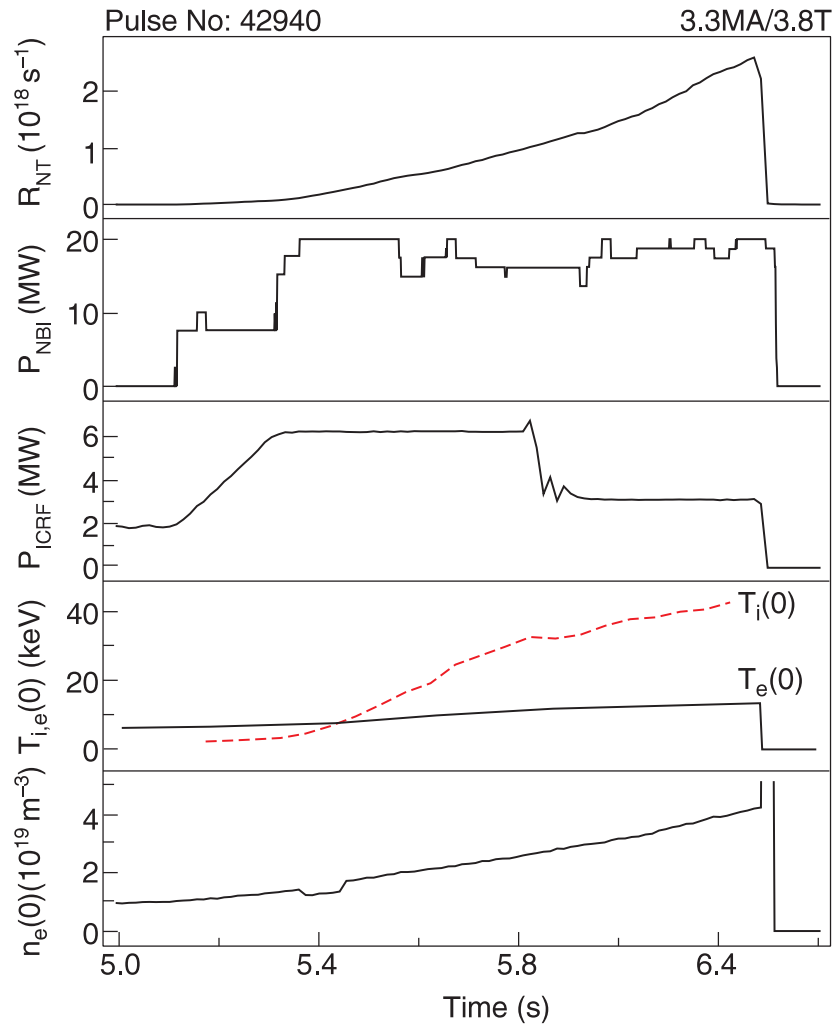

Fig. 14. Temporal evolution of the D-T fusion rate, NBI and ICRF power, central ion (broken line) and electron (solid line) temperatures, and central electron density in D-T discharge 42940 in JET (Ref. 9). 


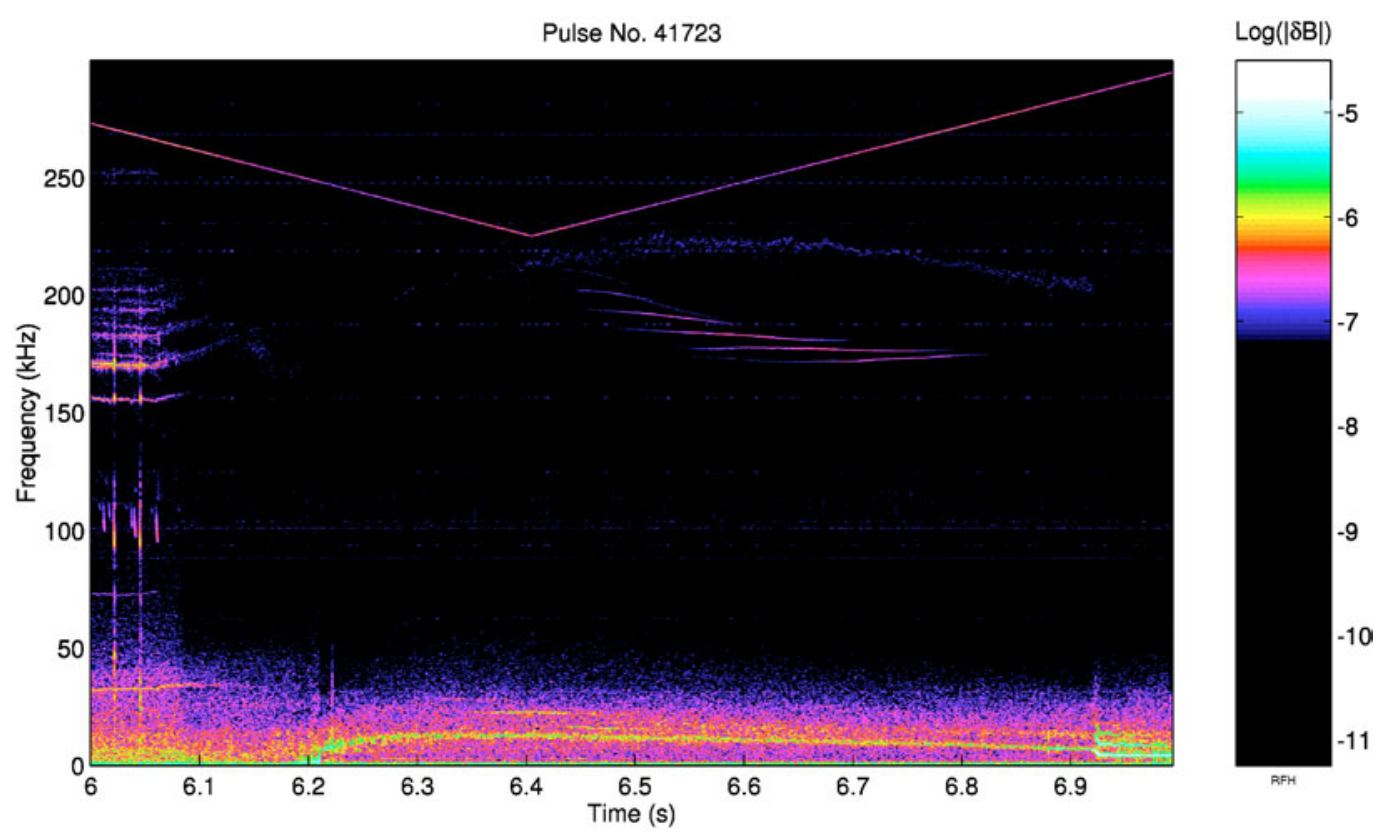

Fig. 15. Spectrogram ${ }^{9}$ of the magnetic perturbations measured by the external Mirnov coils in shear-optimized D-T discharge 41723. The "afterglow" TAEs with toroidal mode numbers ranging from $n=3$ to $n=8$ are seen from $t=6.4 \mathrm{~s}$ to $t=6.8 \mathrm{~s}$.

frequencies in the range of the TAE frequency. The measured modes propagate toroidally in the ion-diamagnetic drift direction (cocurrent) and the mode frequencies follow the Alfvén scaling with plasma density, $f_{0} \propto 1 / \rho_{i}^{1 / 2}$. The detected electromagnetic modes are therefore identified as AEs.

Further analysis of the observed eigenmodes is performed in the same way as for the AEs in hot-ion H-mode discharges. The plasma equilibria are reconstructed for several time slices ranging from $t=5.5 \mathrm{~s}$ to $t=6.2 \mathrm{~s}$. TAEs were then computed by the MISHKA code ${ }^{37}$ for the reconstructed equilibria. The computed TAEs, e.g., at time $t=5.5$, have eigenfrequencies $\omega / \omega_{A}$ as follows: $0.228(n=4), 0.22(n=5), 0.22(n=6)$, and 0.25 $(n=7)$. These eigenfrequencies agree within an accuracy of 10 to $20 \%$ with the experimentally measured ones, which are estimated to be in the plasma reference frame (in units $\left.\omega / \omega_{A}\right): 0.2(n=4), 0.202(n=5), 0.203$ $(n=6)$, and $0.203(n=7)$. All these AEs have a ballooning-type structure of eigenfunctions. A significant part of the eigenmode is radially localized in the region of the maximum gradient of the ICRH-accelerated fast ions, $r / a \approx 0.2$ to 0.4 . These TAEs obtained from the MISHKA code can therefore be identified as the modes displayed in Fig. 13.

AEs driven by ICRH-accelerated energetic ions in optimized shear regimes were found in JET to be more unstable than in the hot-ion H-modes. In particular, in optimized shear D-T discharges, unstable AEs were observed when ICRH in excess of $1 \mathrm{MW}$ is applied. This threshold is much lower than the threshold of $4 \mathrm{MW}$ found for ICRH-driven AEs in H-mode plasmas. The AEs are more unstable in optimized shear discharges due to the low initial plasma density and large slowing-down time of energetic ions, as well as to the high central safety factor $q$, which significantly increases the efficiency of $\mathrm{AE}$ interaction with energetic ions, $\gamma / \omega \propto q^{2}$. Because of the powerful excitation of AEs by ICRH-accelerated energetic ions, no alpha-particle effect on $\mathrm{AE}$ has been detected in high-fusion power optimized shear JET D-T discharges.

\section{III.D. AE Observation in the Afterglow Phase of Optimized Shear Discharges}

In some optimized shear D-T plasmas, AE instability is detected in the afterglow phase of the discharge, approximately 0.5 to $1.0 \mathrm{~s}$ after the auxiliary heating is abruptly switched off. Such a case is shown in Fig. 15. Multiple TAEs driven by ICRH-accelerated energetic ions are seen at frequencies below $200 \mathrm{kHz}$ during the auxiliary heating time, $t<6.1 \mathrm{~s}$. These modes disappear at $t \approx$ $6.1 \mathrm{~s}$, in $<0.2 \mathrm{~s}$ after the ICRH power is switched off, as Fig. 16 shows. After some delay, i.e., 0.3 to 0.7 s later, electromagnetic modes with toroidal mode numbers ranging from $n=3$ to $n=8$ are detected by the Mirnov coils. As can be seen from Fig. 15, the peak fluctuation level of modes at $t \approx 6.5 \mathrm{~s}$ is smaller than that of modes seen at full ICRH power, $t<6.1 \mathrm{~s}$. The observed mode frequencies follow the Alfvén scaling with plasma density, $f_{0} \propto$ $1 / \rho_{i}^{1 / 2}$. Therefore, the modes are identified as AEs. 


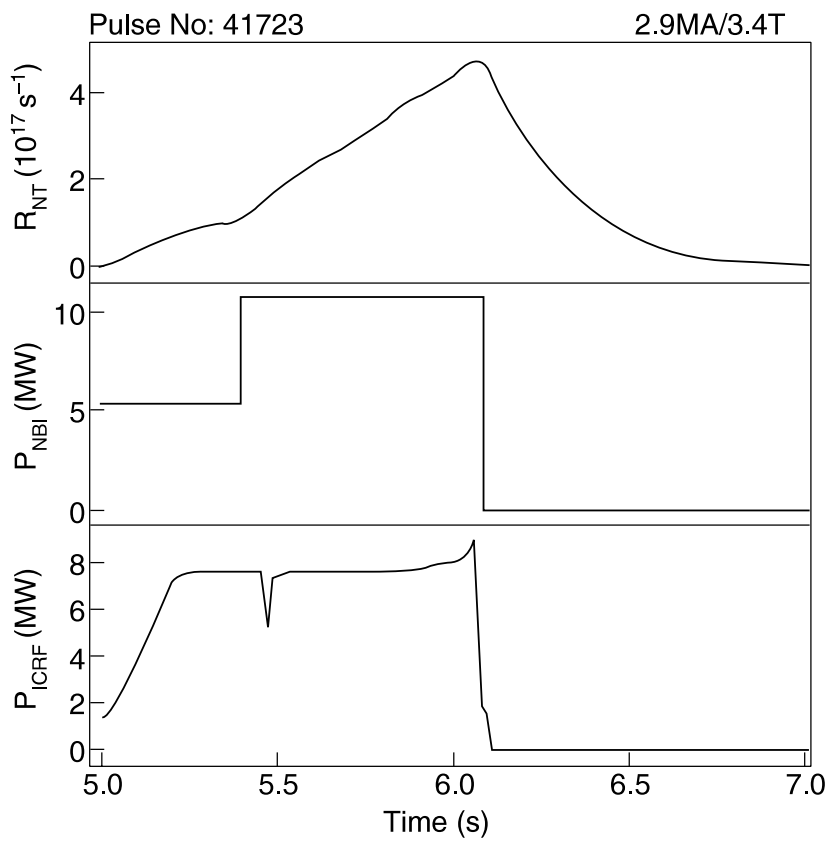

Fig. 16. Temporal evolution of the D-T fusion rate and NBI and ICRF power in shear-optimized D-T discharge 41723 in JET (Ref. 9).

No analogous AE instability was observed in pure deuterium discharges performed prior to the D-T campaign. This indicates that the observed AEs might be driven by alpha particles. Indeed, the analysis of the AE spectrum and the AE drive/damping for the afterglow phase of the optimized shear discharge shows that corelocalized TAEs can exist in this equilibrium and the upper core-localized TAE can be driven unstable by alpha particles (see Fig. 17).

Although the afterglow JET experiments look similar to the experiments on TFTR, where purely alphaparticle-driven AEs were detected, ${ }^{38}$ and although theory confirms the possibility of purely alpha-particle destabilization, the case is not conclusive. The following facts do not allow one to draw a firm conclusion about purely alpha-particle-driven AE instability in JET: First, there are uncertainties in the estimate of the plasma parameters in the afterglow phase of optimized shear discharges in JET, because some key diagnostics cannot operate without NBI. These uncertainties become unacceptably large because the ITB in some cases does not disappear completely in the afterglow phase. Second, the residual drive from ICRH ions, not necessarily trapped, can still compete with the alpha-particle drive, as was shown in TFTR with ICRH power scan in various D-T mixtures. ${ }^{39} \mathrm{~A}$ detailed analysis of the relaxation of ICRH-accelerated ions, however, is complicated by the large error bars in the estimate of the slowing-down time. These error bars are given by the uncertainty in the plasma parameters.

A specific experiment with almost pure deuterium plasma was then performed on JET to assess the possible

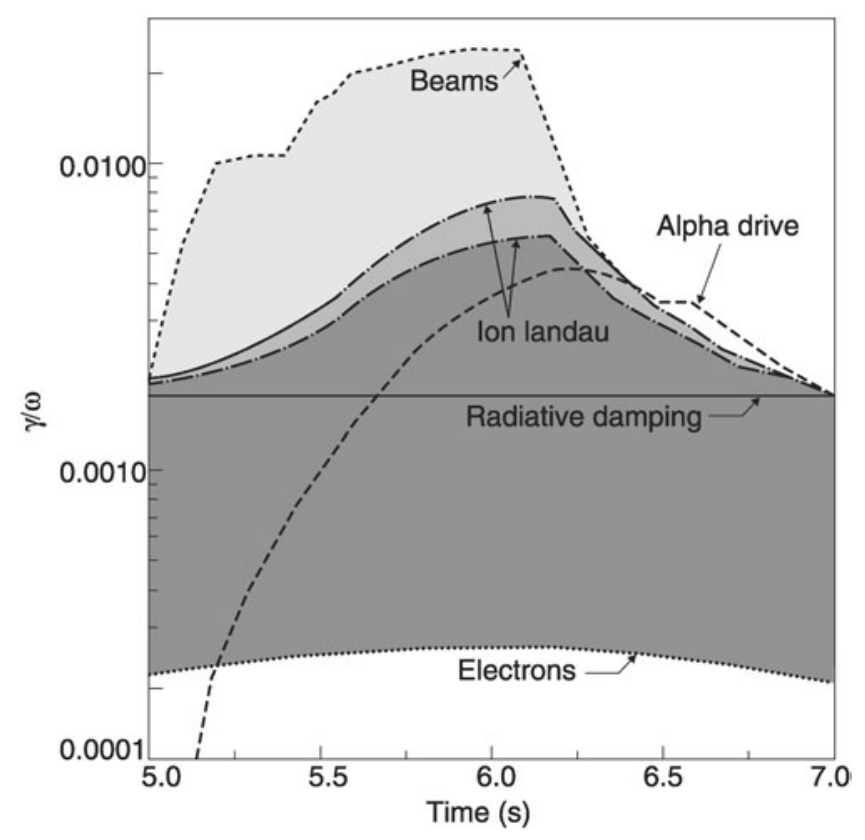

Fig. 17. Temporal evolution ${ }^{9}$ of the alpha-particle drive and the different damping effects computed by the CASTOR-K and the MISHKA code for the $n=6$ upper core-localized TAE in the afterglow phase of shear-optimized D-T discharge 41723. The eigenvalue of the upper core-localized TAE is $\omega / \omega_{A}=$ 0.35 , the dominant poloidal harmonics are $m=9$ and $m=10$, and $q(0)=1.525$.

contribution of ICRH-accelerated ions to the AE drive in the afterglow phase. An AE instability was indeed observed in this almost pure D-D discharge (with D-T fusion power $<0.1 \mathrm{MW}$ ). But the amplitudes of the unstable AEs, $\delta B / B_{0} \ll 10^{-8}$, were close to the limit of diagnostic sensitivity, $\delta B / B_{0} \ll 10^{-9}$, and approximately one order of magnitude smaller than in similar D-T cases.

We conclude therefore that an AE instability seen in the afterglow phase of the optimized shear discharges is possibly a result of the combined excitation by both alpha particles and ICRH-accelerated energetic ions. However, a significant effort in understanding the properties of the transport barrier in the afterglow phase of the discharges is needed to quantify the effect of ICRH-accelerated ions on AEs.

\section{III.E. Conclusions}

Alfvén eigenmodes are found to be stable in highfusion power D-T hot-ion $\mathrm{H}$-mode discharges in JET. The MHD modeling has shown that this result is in agreement with the observed evolution of AEs in deuterium discharges. The model shows that only radially extended and strongly damped KTAEs can exist in high-pressure JET plasmas at the time of high fusion performance. 
In contrast to $\mathrm{H}$-mode plasmas, in optimized shear discharges in JET a significant ICRH power is required for achieving an internal transport barrier. AEs driven by ICRH-heated energetic ions are observed in both JET deuterium and D-T-optimized shear plasmas at all times. No clear effect of fusion-born alpha particles on AEs has been detected so far during the main heating phase of optimized shear discharges.

AEs are sometimes seen in the afterglow phase of the optimized shear D-T discharges, i.e., approximately 0.3 to $1 \mathrm{~s}$ after the auxiliary heating is switched off. These modes were later detected in almost pure deuterium plasma, too. However, the amplitude of the modes observed in deuterium discharges was one order of magnitude smaller than the one in the corresponding D-T discharges. We conclude therefore that in the afterglow phase, AEs are possibly driven by both residual ICRHheated ions and fusion-born alpha particles. Further studies of the internal transport barriers in the afterglow phase are needed to quantify the ICRH drive accurately.

\section{ACTIVE STUDIES OF AEs USING EXTERNAL ANTENNAS}

A burning plasma is characterized by strong nonlinear coupling between its various elements, including the pressure profile, the fusion reactivity, and the alphaparticle phase space distribution. Modern fusion experiments approach this problem by investigating the individual elements of this regime, before actual burning plasma conditions are achieved in ITER. One of these elements is the interaction of alpha particles with waves in the plasma, as discussed in Sec. III.

On the other hand, the knowledge of the mechanisms behind the mode stability, the interaction, and the redistribution can be turned into tools for the control of the alpha-particle population, and hence of the plasma burn. Waves in interaction with alpha particles occupy a relatively empty portion of the plasma electromagnetic fluctuation spectrum, well above the frequencies related to gradient-driven drift instabilities of thermal plasma and well below the gyro frequencies of all plasma species. Therefore, they may constitute a unique way to "communicate" with the core plasma, to extract information on the background plasma and on the alpha particles themselves.

It is therefore very important to assess the physics of this interaction, namely, what modes can be driven unstable, with what efficiency, what determines the subsequent nonlinear evolution and the saturation mechanisms, and, finally, what is the quantitative effect on the alpha-particle distribution. Note that in present and future experiments, other ion species can reach energies of the same order as fusion-born alpha particles, namely, those produced by additional heating, both ICRH and
NBI. Important aspects of the physics of the interaction are common to all fast ion species, although the details of the wave-particle interaction depend on the exact phase space distribution, which is significantly different for these three sources, since fusion-produced alpha particles are mostly isotropic whereas ICRH and, in most cases, NBI ions have large energies primarily in the perpendicular and the parallel directions, respectively. Being the machine closest to producing burning plasma conditions, during the past 15 years JET has been equipped with tools to investigate all of these issues. ${ }^{40}$

In this section, we concentrate on the aspect of the linear stability of the modes that can be driven unstable by the fusion-born alpha particles or by other energetic ions. To obtain quantitative information on the linear properties, namely, on the existence of eigenmodes and their dispersion relation and damping rates, a simple active method to drive and detect low-amplitude modes in the plasma was developed and used in many different plasma conditions. A large database for these quantities for the potentially most important alpha-particle-driven instabilities, eigenmodes in the Alfvén range of frequencies, was constructed. Although in this section we concentrate on the experimental aspects of this research, such results are necessary to benchmark theories and enhance their predictive capabilities.

The section is structured as follows: After a brief description of the active MHD system developed in JET to drive and detect modes in the AE frequency range (Sec. IV.A), Sec. IV.B illustrates the discovery of stable, weakly damped AEs. Sections IV.C and IV.D describe sets of systematic measurements of the AE damping rate as a function of plasma conditions, such as the plasma shape (Sec. IV.C), and quantities that can be used as control parameters, such as the safety factor, the direction of the drift induced by the gradient in the magnetic field intensity, and the plasma rotation. Section VI presents the overall conclusions and an anticipation of future developments.

\section{IV.A. Experimental Setup}

Several requirements were identified for an active MHD system ${ }^{21,22}$ that would be able to drive and detect modes in the $\mathrm{AE}$ frequency range, corresponding roughly from 20 to $500 \mathrm{kHz}$ for JET. First, in-vessel antennas are required. Previously installed saddle coils, dedicated to disruption feedback stabilization and error field control, could be used. Each coil is made of three turns of a large-cross-section Inconel conductor and covers about $90 \mathrm{deg}$ toroidally and $60 \mathrm{deg}$ poloidally, with a selfinductance of about $25 \mu \mathrm{H}$ (Ref. 41).

The exciter system includes a $5-\mathrm{kW}$ broadband power amplifier, an impedance-matching network, a power splitter (distribution unit), and an isolation unit. The power distribution unit can drive different combinations of antenna phasing for one, two, or four saddle coils, 
preferentially exciting specific low toroidal mode numbers (typically $n=0,1$, and 2). Maximum current and voltage applied to the saddle coils are of the order of 20 $\mathrm{A}$ and $600 \mathrm{~V}$. The corresponding magnetic perturbations in the plasma core, $|\delta B / B|<10^{-6}$, are not expected to significantly enhance the transport of energetic particles. As an active system injecting significant voltage levels, the active MHD exciter is interfaced with the main JET machine and personnel safety networks. Ground faults and turn-to-turn short circuits can be detected over a fast time scale $(<1 \mathrm{~ms})$ and used to trip the power amplifier to avoid sustaining arcs during plasma operation. Crowbars are activated during plasma disruptions and their signal is also used to inhibit the $5-\mathrm{kW}$ amplifier output.

The plasma response ${ }^{42-44}$ during repetitive sweeps of the driving frequency is determined in terms of driven oscillating quantities such as magnetic fields and densities, extracted from background noise via synchronous detection of signals from magnetic coils, ECE, heterodyne reflectometer, and X-ray fast cameras. Synchronous detection is performed by analogy. The diagnostic signals are multiplied by a reference signal and then are low-pass filtered. The reference signal typically corresponds to the injected current and is transmitted optically to the different areas of the plant where the diagnostic signals are acquired. The in-phase and quadrature signals result from the multiplication with the reference signal itself and with its 90 deg out of phase component and, once properly normalized (typically to the current driven in the saddle coils), correspond to the real and imaginary parts of the antenna-plasma transfer function $H(\omega, r)$. The transfer function $H(\omega, r)$ is digitized at $1.5-\mathrm{kHz}$ rate for $12 \mathrm{~s}$, allowing for a full coverage of the plasma discharge. With recent improvements in the direct digitization of the fluctuating signals, in terms of sampling rate (up to $2 \mathrm{MHz}$ at present), dynamic range (14 bits), and memory depth, synchronous detection can presently also be performed on the digital signals.

By fitting the measured transfer function $H(\omega, r)$ for a number of probes with a rational function $H(\omega, r)=$ $B(i \omega, r) / A(i \omega)$, one obtains the mode frequency, amplitude, damping rate $\gamma / \omega$, and radial structure in different regimes as a function of various plasma parameters. ${ }^{45}$ The time resolution of the frequency and damping measurements can reach $10 \mathrm{~ms}$, for a frequency resolution of less than $100 \mathrm{~Hz}$ and frequency sweep rates of the order of $200 \mathrm{kHz} / \mathrm{s}$.

\section{IV.B. Demonstration of the Existence of Stable, Weakly Damped AEs}

Since the very first experiments using the JET active MHD system, ${ }^{21,42}$ the existence of stable AEs of weak damping $(\gamma / \omega<10 \%)$ was very clearly demonstrated, confirming MHD theory to a high degree of accuracy. Figure 18 shows an example of a plasma resonance corresponding to an $n=1 \mathrm{TAE}$. In the same figure, a clear

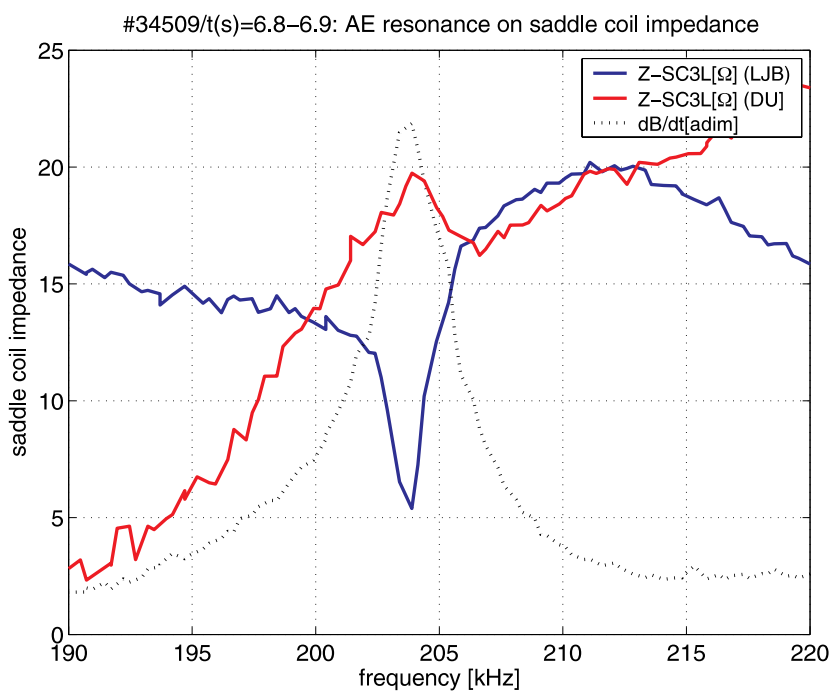

Fig. 18. Single resonance with complex circle, and peak in the antenna loading, as measured at two different points along the power transmission line: at the Distribution Unit output (labeled DU), located about $80 \mathrm{~m}$ before the antenna (long coaxial cable), and at the limb junction box (labeled LJB), located much closer to the antenna, about $10 \mathrm{~m}$ before the feedthrough. For clarity the antenna loading only for one saddle coil (SC3L) is shown, with very similar results for the other active antenna (SC7L). Note that the antenna loading increases at the DU and decreases at the LJB when a resonance is found in the plasma.

signature of the $\mathrm{AE} /$ resonance is visible on the impedance signal, measured at two different points along the power transmission line. An eigenmode corresponds to a peak in the antenna loading (typically causing an increase/ decrease in the loading of up to a few ohms). Note that this parameter is the most practical way of detecting the eigenmodes in numerical simulations, assessing the coupling and even determining their damping rates. In fact, in the codes a virtual antenna is inserted and its loading as a function of frequency is computed. ${ }^{43}$

These data confirm that modes of global nature can be coupled with external antennas and that extremely high signal-to-noise ratios can be achieved. The neatness of the plasma response is due to the measurement technique, to the robustness of the MHD modes in the plasma, and to the fact that the plasma spectrum is exempt from high-activity turbulence levels in this range of frequency (see Fig. 19). A very accurate determination of the mode parameters (frequency, structure, and damping rate) ${ }^{21}$ is therefore possible, opening the way, more generally, for a very precise "MHD spectroscopy." 44

The potential of the AE active diagnostic is significantly enhanced by the application of a digital real-time control system to perform individual resonance tracking. The controller of the exciter frequency, run at a 1-ms 


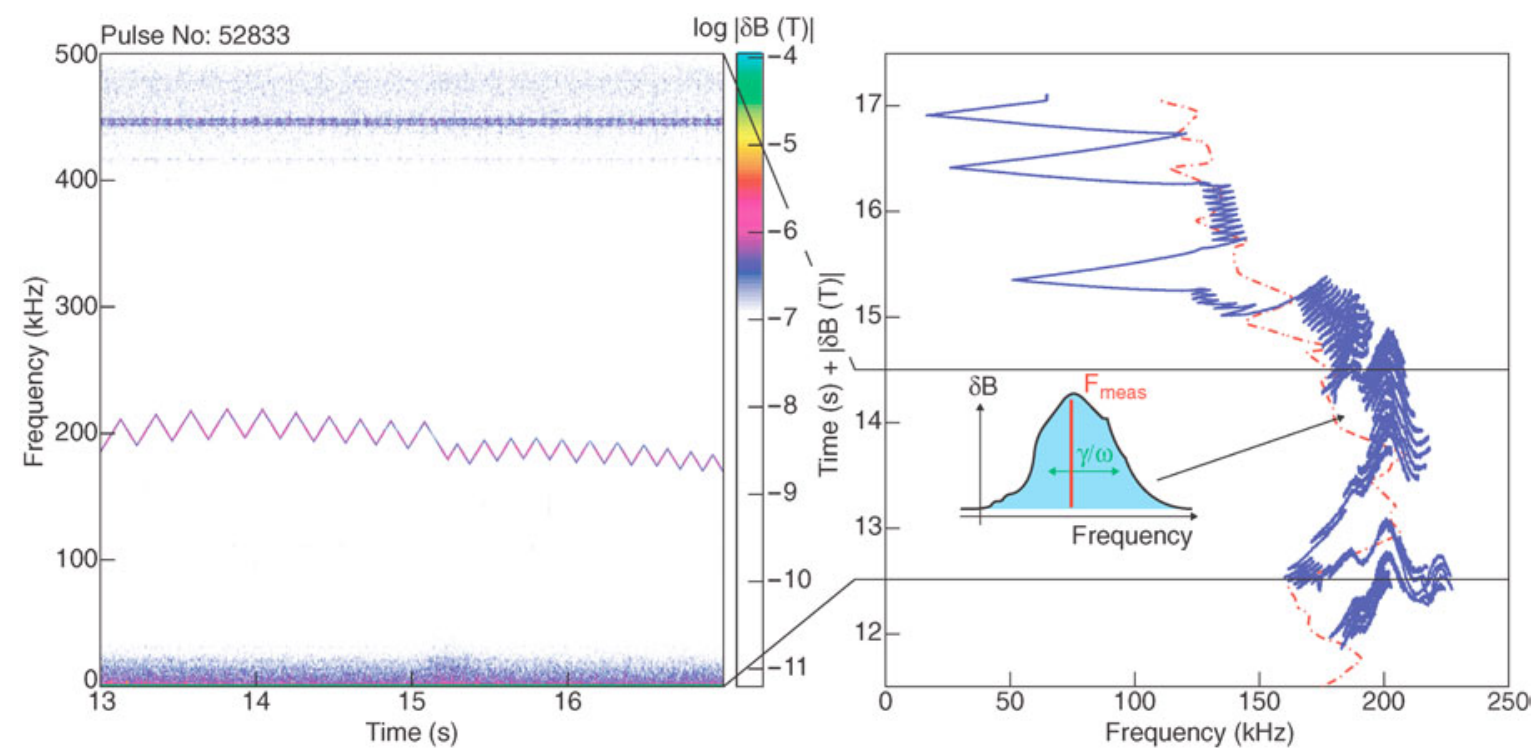

Fig. 19. Tracking of an individual AE (an $n=1$ TAE) in the absence of fast particle drive in the limiter phase of a JET ohmic discharge, with the synchronously detected signal (right), shown using a false three-dimensional representation, and the spectrogram of the directly digitized magnetic perturbation (left). ${ }^{44}$ Note the complete absence of background fluctuations in the AE frequency range for ohmic plasmas (left), where the antenna frequency is rapidly swept.

clock rate, varies the frequency linearly around the expected frequency of the mode $f_{\mathrm{AE}}$, calculated in real time from the measured density and magnetic field. The frequency $f_{\mathrm{AE}}$ corresponds to the center of the gap for the chosen class of eigenmodes, which is used as a first guess for the exact value of the eigenfrequency and should be calculated from the local value of the density, magnetic field, and safety factor. In most experimental conditions, for the low- $n$ modes driven by the saddle coils, the lineintegrated density divided by the major radius is used, together with $q=1.5$. A frequency multiplier that is assumed to be proportional to the total plasma current is used to account for the variation of the safety factor within a discharge.

When an $\mathrm{AE}$ resonance is met, the exciter frequency is swept back and forth around the resonance full width. As a result, the AE frequency and damping rate can be measured in real time with high time resolution (typically $<50 \mathrm{~ms}$ ) along their dynamical evolution throughout a JET discharge, as shown in Fig. 19. Discrimination of the toroidal mode numbers ( $n$ odd versus $n$ even) in the real-time detection is also possible. We have also developed a simple algorithm to obtain in real time the mode frequency $f_{\mathrm{AE}-\mathrm{RT}}$ and the damping rate $(\gamma / \omega)_{\mathrm{AE}-\mathrm{RT}}$ from the width of the Alfvén eigenmode local manager frequency sweep, using the phase inversion characteristic of a stable, antenna-driven, plasma resonance. The realtime measurements are in very good agreement with the computed/fitted values. ${ }^{45}$

Stable TAEs, EAEs, and NAEs are routinely driven and detected in JET ohmic plasmas in the range 80 to 500
$\mathrm{kHz}$, with measured damping rates covering a large range, $10^{-3}<\gamma / \omega_{\text {damp }}<10^{-1}$. From the time evolution of the frequency of the driven and tracked mode, information on parameters other than the AE characteristics themselves, but which depend on them, such as the isotopic mass or the value of the product $n_{e} q^{2}$ ( $n_{e}$ is the electron density) at the eigenmode resonance location, can also be reconstructed.

\section{IV.C. Damping Rate of Global AEs as a Function of the Plasma Shape}

Systematic studies are needed to reveal the dependence of the AE damping rate on the plasma parameters. To provide an accurate testing for the code predictions, it is crucial that the same mode be measured throughout the parameter scan. For this, real-time tracking of the antennadriven modes constitutes in practice an invaluable tool.

In JET discharges that evolve from a limiter to a diverted configuration, the damping rate of the antennadriven low- $n$ AEs starts relatively low and increases dramatically when the plasma approaches the $X$-point phase and the edge magnetic shear is increased. ${ }^{45}$ As the $X$ point is formed in the plasma, the damping becomes too large $(\gamma / \omega>5 \%)$ for the resonance to be recognized in real time. This experimental observation has motivated a significant amount of theoretical and modeling work. ${ }^{46,47}$ These comparisons indicate that strong damping can occur in regions of large magnetic shear at the plasma edge and suggest a strong sensitivity to the plasma shape. Systematic experimental analyses of $\mathrm{AE}$ damping rates as a 
function of the plasma shape and edge magnetic shear were conducted for radially extended $n=1$ TAEs and $n=$ 0 global Alfvén eigenmodes (GAEs) in ohmic limiter discharges $^{45}$ and for core-localized $n=3$ to 10 TAEs driven by resonant ions in limiter and $X$-point discharges ${ }^{48}$

Figure 20 (left ${ }^{45}$ shows the dependence of the damping rate separately on the core $\left(\kappa_{0}\right)$ and edge $\left(\kappa_{95}\right)$ elongation, and on the lower $\left(\delta_{L O W}\right)$ and upper $\left(\delta_{U P}\right)$ triangularity. The data for the elongation scan were averaged over $0.08<\left[\delta_{L O W}, \delta_{U P}\right]<0.12$, and those for the triangularity scan were averaged over $1.1<\kappa_{0}<1.2$ and $1.2<\kappa_{95}<1.3$. We notice that when averaging over low values of the plasma elongation, the $\mathrm{AE}$ damping rate increases approximately linearly with the triangularity and the edge magnetic shear. Conversely, when averaging over low values of the plasma triangularity, the $\mathrm{AE}$ damping rate shows a sharp increase for a small variation in the elongation around $\kappa_{95} \approx 1.5$ and $\kappa_{0} \approx 1.35$. Figure 20 (right) ${ }^{43}$ summarizes the results obtained in the experiments for stable low- $n$ AEs as a function of the mean triangularity $\langle\delta\rangle=\left(\delta_{L O W}+\delta_{U P}\right) / 2$ and of the midradius elongation $\kappa(r / a=0.5)$, which is obtained with a parabolic fit $\kappa=\kappa_{0}+\alpha(r / a)^{2}$, with $\alpha=\left(\kappa_{95}-\kappa_{0}\right) /$ $(0.95)^{2}$. These measurements show a strong effect of the edge plasma shape, as determined by the elongation and triangularity, on the AE damping rate: The effect is comparable for the $n=0$ GAEs and the $n=1$ TAEs.

Results along the same line were also obtained for the case of $n=3$ to 10 TAEs excited by resonant NBIproduced ions, with $v_{\| \mathrm{NBI}} \approx v_{\mathrm{A}}=B /\left(\mu_{0} \rho\right)^{1 / 2}$ (here $\rho$ is the plasma mass density), by comparing limiter and
$X$-point plasma configurations (i.e., low and high edge magnetic shear, respectively). ${ }^{48}$ For the $X$-point scenario, a ramp in the NBI heating power is used to determine the TAE instability threshold, whereas for the limiter configuration various discharges were run at different NBI power levels due to the limited power-handling capability of some in-vessel protection tiles. For similar background plasmas, we find that one needs approximately $50 \%$ more NBI power $\left(P_{\mathrm{NBI}}=8 \mathrm{MW}\right.$ compared to $P_{\mathrm{NBI}}=$ 5.3 MW) and NBI fast ions with parallel velocity much closer to the resonant velocity $\left[\max \left(v_{\| \mathrm{NBI}}\right) \approx 0.95 v_{\mathrm{A}}\right.$ compared to $\left.\max \left(v_{\| \mathrm{NBI}}\right) \approx 0.8 v_{\mathrm{A}}\right]$ to destabilize TAEs with intermediate $n$ 's in plasmas with high edge magnetic shear than in plasmas with low edge magnetic shear.

These measurements clearly indicate the role of the edge magnetic shear in the damping of AEs whose radial structure has a significant edge component, since the large edge magnetic shear acts as an energy sink for these modes since it causes a strong interaction with the edge Alfvén continuum. A similar effect was recently found to be important in modeling global AEs driven in ITER (Ref. 49). This idea was further tested experimentally ${ }^{50}$ by measuring the damping rate of antenna-driven $n=1$ EAEs. Contrary to the TAE cases, these modes typically interact with the Alfvén continuum only toward midradius (usually around $r / a \approx 0.65$ ), and their damping does not depend significantly on the edge shape and magnetic shear. This clearly indicates that when the source of the damping is not localized at the edge of the plasma, the damping of the modes is independent of the edge plasma shape.
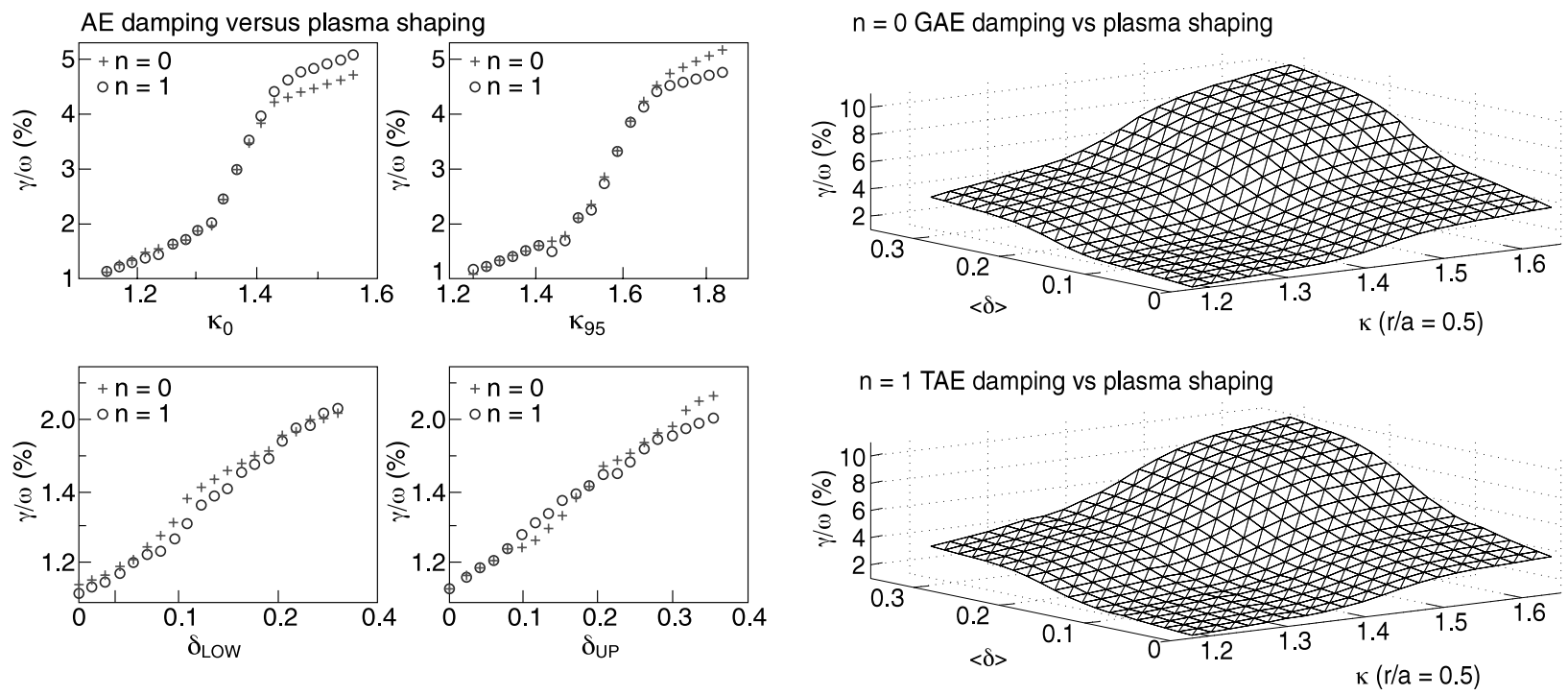

Fig. 20. Variation of the measured AE damping rates as a function of the elongation (at almost constant $0.08<\delta_{L O W} \approx \delta_{U P}<0.12$ ) and triangularity (at $1.1<\kappa_{0}<1.2$ and $1.2<\kappa_{95}<1.3$ ), for $q_{95} \approx 4, q_{0} \approx 0.9$, and $\sigma_{0} \approx 1$. Here $\langle\delta\rangle=\left(\delta_{U P}+\delta_{L O W}\right) / 2$ and $\kappa(r / a=0.5)$ is given by the parabolic fit $\kappa=\kappa_{0}+\alpha(r / a)^{2}$. The data are shown for $n=0$ GAEs and $n=1$ TAEs during the ohmic limiter phase of JET plasma pulses. We note a sharp variation in $\gamma / \omega$ as a function of the elongation around $\kappa_{95} \approx 1.5$ and $\kappa_{0} \approx 1.35$, whereas $\gamma / \omega$ varies approximately linearly with the triangularity. ${ }^{45}$ 
The strong sensitivity of the damping rate of radially extended AEs upon the edge shape parameters may lead to a method to control the stability of fast ions resonating with AE global wave fields. Based on a command generated from the real-time measurement of the AE damping rate, the coils used to shape the plasma could be energized with a typical time response of the order of $20 \mathrm{~ms}$, thus achieving a real-time control of radially extended, fast ion-driven AE instabilities.

\section{IV.D. Damping Rate of Global AEs as a Function of Plasma Parameters}

Having established the role of the plasma shape in determining the AE damping, and hence the AE stability limits, experiments have been performed in JET to identify the dependence of the $\mathrm{AE}$ damping rate on a variety of plasma parameters. These investigations have a twofold aim: to contribute to a better understanding of the AE stability physics, in order to improve our predictive capability, in conjunction with theory and numerical modeling, and to identify which operational parameters can be used in practice to control the AE stability in burning plasmas such as ITER.

The experiments were designed to avoid being dominated by edge damping, and explore other mechanisms and their scaling. In particular, the strong increase of $\gamma / \omega$ for the saddle coil-driven, stable low- $n$ AEs at high edge elongation $\left(\kappa_{95}>1.5\right)$ and triangularity $\left(\delta_{95}>0.35\right)$ makes it difficult to measure the damping rate for $n=1$ AEs in such configurations, since in these cases $\gamma / \omega \approx 10 \%$ and the broadening of the AE resonance makes it difficult to fit the transfer function. Hence, our experimental work was mainly focused on plasmas with low $\kappa_{95}$ and $\delta_{95}$, in which the measured $\gamma / \omega$ is smaller. We provide here some examples of these parameter scans and of the conclusions that can be drawn from the relevant databases.

A set of plasma discharges with different isotopic mass, during D-D and D-T campaigns, were investigated. The measured low- $n$ TAE damping rate was found to decrease with the isotopic mass. Such scaling, as well as the absolute value of the damping, appeared to be incompatible with fluid theory, which predicted much lower damping rates. ${ }^{46}$ This indicated an incomplete description of the mode-plasma interaction and motivated a set of dedicated experiments specifically designed to test the predictions of a theory model for a specific damping mechanism, referred to as radiative damping. ${ }^{51}$

Among the various mechanisms that have been proposed to account for the AE damping rate, the radiative damping model gives a clear dependence on the ion Larmor radius $\rho^{*}$ and the $\lambda$ parameter, $\lambda \propto \sigma \rho^{*}(3 / 4+$ $\left.T_{e} / T_{i}\right)^{1 / 2},(\gamma / \omega)_{R A D} \propto \exp \left(\sigma^{2} / \lambda\right)$. The measured variation of $\gamma / \omega$ for an $n=1$ TAE as a function of $\rho^{*}$ and $\lambda$ is shown in Fig. 21 for a series of discharges characterized by a weak core magnetic shear. Here the magnetic field and plasma current were increased at fixed $q_{0} \approx 0.8$ and $q_{95} \approx 2.8$, to change $\rho^{*}$, and $\lambda$. These results clearly show that $(\gamma / \omega)_{M E A S} \approx 10 \times(\gamma / \omega)_{R A D}$, and $(\gamma / \omega)_{R A D}$ predicts a different scaling for increasing $\rho^{*}$ and $\lambda$. We conclude that the radiative damping model is not sufficient to predict correctly the AE stability limit. ${ }^{52-54}$

As part of the investigations conducted to identify the dependence of the AE damping on operational control parameters, we measured the TAE damping rate in a
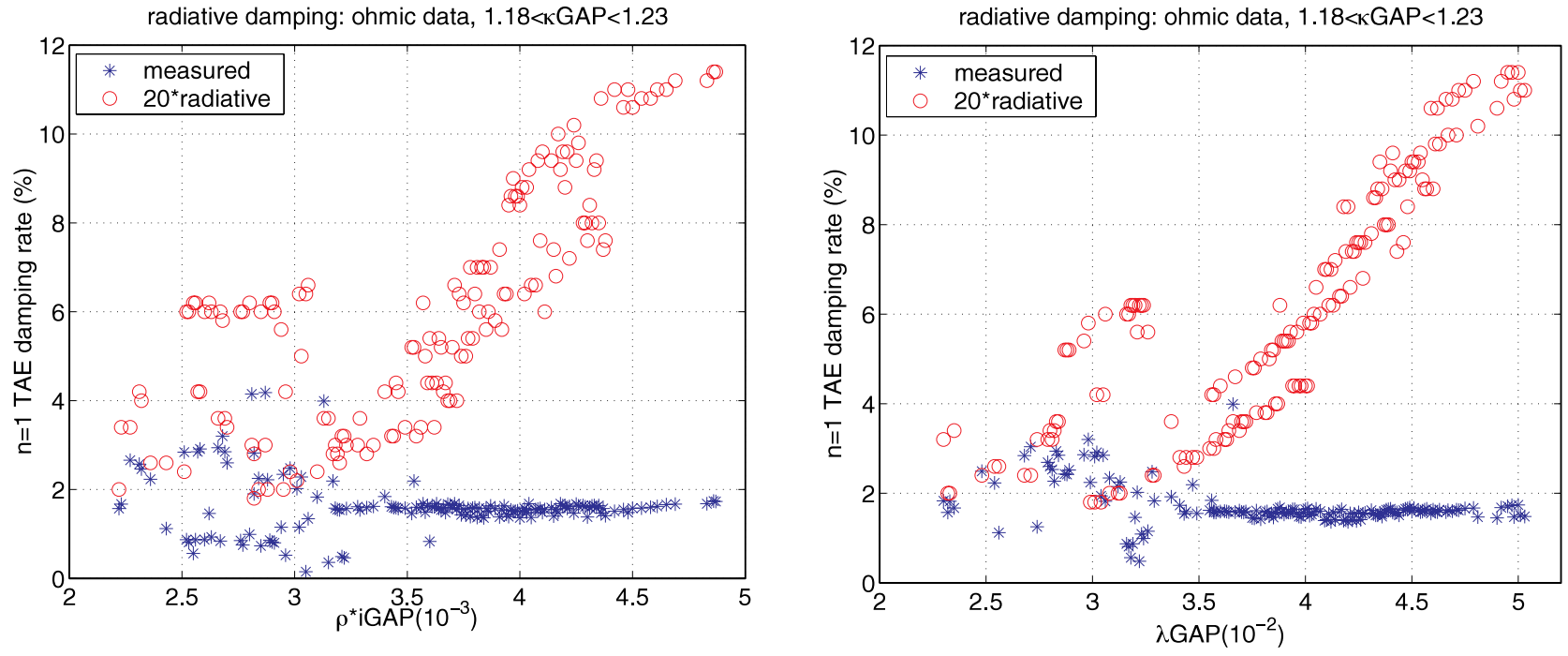

Fig. 21. The dependence on $\rho^{*}$ and $\lambda$ (estimated at $q=3 / 2$ ) of the $n=1$ TAE damping rate measured and computed using the radiative damping model for a series of discharges with similar plasma parameters ${ }^{52}: T_{e 0} \approx(0.8$ to 2.6$) \mathrm{keV}, n_{e 0} \approx$ $(1.8$ to 3$) \times 10^{19} \mathrm{~m}^{-3}, T_{i 0} \approx(0.6$ to 2.3$) \mathrm{keV}$, and $\delta \approx 0.05$. 
large set of discharges with different values of the safety factor on axis, in ohmic limiter configuration with a monotonic $q$ profile. ${ }^{55}$ A database containing more than 1500 points (from 23 individual discharges) was constructed, with $q_{0}$ in the range $0.76<q_{0}<1.59$. This database covers a wide range in the main plasma parameters [edge elongation and triangularity $1.24<\kappa_{95}<1.55$ and $0<$ $\delta_{95}<0.25$, central electron density and temperature $1.35<$ $n_{e 0}\left(10^{19} \mathrm{~m}^{-3}\right)<4.2$ and $1.1<T_{e 0}(\mathrm{keV})<5.6$, and edge safety factor $\left.2.5<q_{95}<4.75\right]$, and hence represents a significant statistical overview of the damping rate measurements in this configuration. The $q$ profile is reconstructed by the EFIT code using edge magnetic and internal polarimetry measurements, with a typical accuracy of the order of $10 \%$ for the cases considered here. Furthermore, for $q_{0}<1$, a very good agreement was found between the position of the $q=1$ surface as determined by EFIT and from the sawtooth inversion radius. Figure 22 shows the dependence of the measured damping rate for $n=1$ TAEs on $q_{0}$. Considering ohmic plasmas with $\kappa_{95}<1.5$ and $\delta_{95}<0.3$, we find that for $q_{0}>1.1$ the damping rate of $n=1$ TAEs does not exceed the value $\gamma / \omega \approx 2 \%$ for a variety of electron temperature and density profiles and for different values of the plasma current and magnetic field. For $q_{0}>1.1$, experimental conditions were not found for which the $n=1 \mathrm{TAE}$ damping rate can be made large enough for the modes to be stable against the typical fast ion drive computed for JET plasmas with a monotonic $q$ profile and low edge magnetic shear. On the other hand, we find that with $q_{0}<$ 0.9 in otherwise similar experimental conditions, $\gamma / \omega$ can reach values up to $\gamma / \omega \approx 8 \%$. Such observations

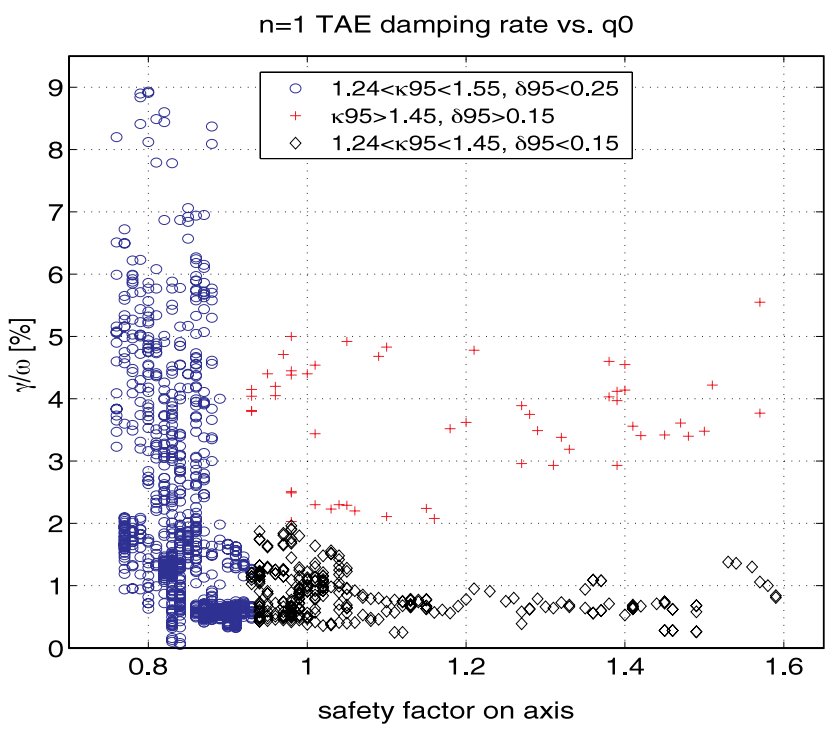

Fig. 22. The scaling ${ }^{53}$ of the measured damping rate for $n=1$ TAEs versus $q_{0}$. Note that for $q_{0}>0.95$, a high value of the damping rate, $\gamma / \omega>2 \%$, can be obtained only at high $\kappa_{95}$ and $\delta_{95}$. indicate that large values of the damping rate $(\gamma / \omega>$ $2 \%$ ) can be obtained for $q_{0}<1$ only in plasmas with a low magnetic shear, which are then less prone to becoming TAE unstable than similar plasmas with $q_{0}>1.1$. Detailed theoretical modeling is necessary to reproduce and provide an interpretation for this phenomenon. The separation between these two regimes, observed for $q_{0} \approx$ 0.9 to 1.1 , is suggestive of a role for the $q=1$ surface and possibly for the sawteeth, for example, an average effect of the sawteeth redistribution of the plasma current providing, on average, a lower magnetic shear in the plasma core, and hence favoring core damping via mode conversion.

Two parameters that are important both in terms of a direct effect on the plasma characteristics, well beyond the AE stability properties, and for identifying the necessary ingredients that a numerical model should include to correctly represent the AE stability limits, are the plasma rotation and the magnetic field direction. Theoretical modeling and direct measurements ${ }^{52,54}$ indicate that low- $n$ AEs have a global structure, i.e., have a radial profile extending over a large fraction of the plasma cross section. The shear in the toroidal rotation $f_{R O T}$ of the whole plasma column, $s_{R O T}=\left(r / f_{R O T}\right)\left(d f_{R O T} / d r\right)$, can thus be expected to have an impact over the effective mode damping. The measurement of the different effect of the shear in the toroidal rotation profile on the damping rate of $n=1$ TAEs as a function of $P_{\mathrm{NBI}}$ provides empirical indications that different damping mechanisms are active at low and high performance. ${ }^{53}$ Similar to the $q_{0}$-scan case, detailed theoretical modeling using codes that include explicitly the sheared toroidal rotation profile is needed to reproduce these observations.

In JET ohmic limiter discharges, for very similar background plasma parameters and eigenfunctions, the damping rate of $n=1$ TAEs is measured to be a factor of 3 larger when the magnetic field and plasma current are reversed together, i.e., when the ion $\nabla B$ drift is directed away from the divertor. ${ }^{48} \mathrm{~A}$ theoretical explanation for these measurements is still lacking, other than the simple observation that ion $\nabla B$ drift terms are present in gyrokinetic codes, whereas fluid codes include only equilibrium gradients depending on the density and temperature. However, we also note that in JET the plasma helicity is conserved when reversing the toroidal magnetic field (i.e., the toroidal plasma current is also correspondingly reversed), and the limiter discharges used for the measurement of the $n=1 \mathrm{TAE}$ damping rate were largely up-down symmetric. Hence, in principle there could be only a minimal effect coming from the specific ion $\nabla B$ drift terms present in the gyrokinetic codes because of these dominant up-down symmetries. On the other hand, the difference in the measured $\gamma / \omega$ for $n=1$ TAEs could be associated with the observation of different flows at the plasma edge and scrape-off layer for experiments with forward and reversed magnetic field, and hence with the ion $\nabla B$ drift directed to and away from the divertor, 
respectively. ${ }^{55}$ In addition to a possible direct effect on the TAE damping rate, these flows also change the e-folding length for the edge density from the scrape-off layer toward the first wall, hence possibly affecting the interaction of the TAE modes with the Alfvén continuum at the plasma edge.

\section{IV.E. Conclusions and Outlook}

Several years of experiments using an active antenna system to drive and detect modes in the Alfvén frequency range in JET have produced a large variety of experimental results. The existence of weakly damped, stable eigenmodes, clearly identified as AEs, was demonstrated, in accordance with MHD theory. No other resonant modes were found in the plasma spectrum in the Alfvén wave frequency range (20 to $500 \mathrm{kHz}$ ) in the absence of energetic particles. Of direct interest for predicting the linear stability limits of modes interacting with energetic ions, including fusion-produced alpha particles, in ITER and other future experiments in the burning plasma regime, is the large database that was constructed with measurements of the AE damping rate in a variety of plasma configurations.

A clear stabilizing effect of the magnetic shear close to the plasma edge was observed for the antenna-driven low- $n$ AEs. Such a mechanism provides large damping rates and can be used via appropriate plasma shaping to establish a region free of unstable AEs, in which fast ion transport induced by resonant AEs would be prevented.

A particular effort was devoted to establishing parametric dependencies in the experiment to provide stringent tests for the theory, despite an extreme sensitivity in the theoretical predictions of the damping rates to the details of the plasma equilibrium profiles. For damping mechanisms acting in the plasma core, several challenges were posed to the theory. For example, the model of radiative damping is demonstrated to be inadequate for low- $n$ TAEs, and the role of edge flows, of the plasma rotation and its shear, appear to be important in the experiment and require a rather comprehensive treatment beyond the MHD model.

Although modes generally appear to be more strongly damped in the experiment than in theoretical predictions, the lack of agreement across the whole range of plasma conditions still limits our predictive capabilities for ITER and future reactors. An effort is required on the theory side to systematically compare with the data the different models of increasing complexity and identify the necessary ingredients for reproducing the experimental results on the mode damping rates.

On the experimental side, due to the saddle coil geometry, the intermediate $n(n=3$ to 15$)$ range could not be explored in JET experiments to date. Since the most unstable modes for ITER are expected to belong to this range, a new dedicated set of in-vessel antennas for JET was designed and constructed. To excite different com- binations of high- $n$ AEs, eight antennas asymmetrically located in the toroidal direction have been built. Each antenna has a small solenoid-like shape with 18 turns, corresponding to a static self-inductance for each antenna of the order of $80 \mu \mathrm{H}$. The new AE antennas are divided into two groups of four antennas each, positioned as close as possible to the plasma, with a distance between the first turn of the antenna and the last closed flux surface of the order of $6 \mathrm{~cm}$. A single group of four antennas has been installed in JET and has recently started to produce experimental results.

Together with the excitation of higher toroidal mode numbers, more effort in internal mode structure reconstruction will be required. Because several modes can be present at different radial positions in the intermediate $n$ regime, some knowledge of the mode structure is essential for the choice of which mode should be considered in comparisons with theory. Comparisons with other experiments adopting the same method as JET to launch and detect modes in the Alfvén frequency range and directly measure their damping rate will also significantly contribute to our capability of identifying and quantifying the dominant damping mechanisms in ITER, possibly leading to an optimization of operation scenarios in terms of the stability of alpha-particle-driven modes.

\section{DEVELOPING NEW DIAGNOSTIC TECHNIQUES FOR BURNING PLASMA}

Diagnosis of energetic ions and energetic ion-driven instabilities is of major importance for burning plasma devices, ${ }^{1,56}$ so the development of burning plasma diagnostics is one of the most important problems for the magnetic fusion. This problem is challenging, too, since the burning plasma diagnostics under the harsh conditions of D-T plasma must be capable of performing accurate simultaneous measurements of several populations of energetic ions: fusion-born alpha-particles, NBIproduced $\mathrm{D}$ ions in the $\mathrm{MeV}$ energy range, and minority ions accelerated with ICRH (Ref. 1). In addition, reliable techniques for detecting electromagnetic plasma oscillations coupled to some of these energetic ions via the wave-particle resonances, such as fishbones ${ }^{57}$ and AEs (Ref. 30), are also required since these perturbations may affect transport and losses of the energetic ions.

This section reviews the development and testing of diagnostic techniques measuring distribution functions of energetic ions and energetic ion-driven instabilities in JET during the decade 1996 to 2006. The main diagnostic techniques are reviewed here: measurements of the distribution function of energetic ions with a neutral particle analyzer in Sec. V.A, diagnosis of the confined alpha-particle population derived from the neutron emission spectrum in Sec. V.B, gamma-ray spectroscopy and gamma-ray tomography in Sec. V.C, gamma-ray 
measurements of fusion-born alpha particles in trace tritium experiments in Sec. V.D, interferometry/ reflectometry detection of fast ion-driven Alfvén cascade eigenmodes in reversed shear JET discharges in Sec. V.E, simultaneous time-resolved measurements of ICRH-accelerated ${ }^{3} \mathrm{He}$ ions with $E>500 \mathrm{keV}$ and coupled energetic ion-driven AEs in Sec. V.F, and further improvements of fast ion diagnostics in JET in Sec. V.G.

\section{V.A. Measurements of the Distribution Function of Energetic Ions with a Neutral Particle Analyzer}

Direct detection of energetic ions in JET is carried out with the help of the neutral particle analyzer (NPA) and relies on neutralization of the ions in CXS reactions with intrinsic impurities: carbon, beryllium, and helium (the last is present when helium NBI fueling is employed). This effect is called impurity-induced neutralization (IIN) and it is described in Ref. 58 and references therein. High efficiency of IIN for the energetic ions results from closeness of their velocities to the orbital velocities of the electrons in the ground states of main $[\mathrm{H}]$ and $[\mathrm{He}]$ impurity ions. The most important reactions for hydrogen isotope ions are

$$
\begin{aligned}
& \mathrm{H}^{+}\left(\mathrm{D}^{+}, \mathrm{T}^{+}\right)+\mathrm{C}^{5+}, \mathrm{Be}^{3+}, \mathrm{He}^{+}, \mathrm{C}^{4+}, \mathrm{Be}^{2+}, \mathrm{He} \\
& \quad \rightarrow \mathrm{H}(\mathrm{D}, \mathrm{T})+\mathrm{C}^{6+}, \mathrm{Be}^{4+}, \mathrm{He}^{2+}, \mathrm{C}^{5+}, \mathrm{Be}^{3+}, \mathrm{He}^{+} .
\end{aligned}
$$

Three key factors determined a successful development of the diagnostic based on IIN:

1. development of an asymptotic theory of nonadiabatic transitions relevant to the above reactions

2. development and employment of a special NPA GEMMA-2 capable of measurements of hydrogen and helium isotope fluxes in the energy range $0.3 \leq E(\mathrm{MeV}) \leq 3.5$ with close to $100 \%$ detection efficiency and very low sensitivity to gamma and neutron radiation $\left[\sim 10^{-7}\right.$ at $E \geq 1 \mathrm{MeV}$ (Ref. 59)]

3. reliable measurements of the local density of bare impurity ions provided by CXS spectroscopy. ${ }^{60}$

The NPA measurements are intended to

1. provide experimental testing and validation of theories of radio frequency heating and heating scenarios

2. quantify the link between energetic ions and MHD instabilities

3. investigate confinement and slowing down of charged fusion products.

The NPA is located at the top of the torus, with its vertical line of sight intersecting the ICRH power deposition region and lower energy (octant 4) NBI at the plasma center. ${ }^{58}$ The energy distribution function $f(E)$ integrated along the line of sight is measured for ions in the narrow

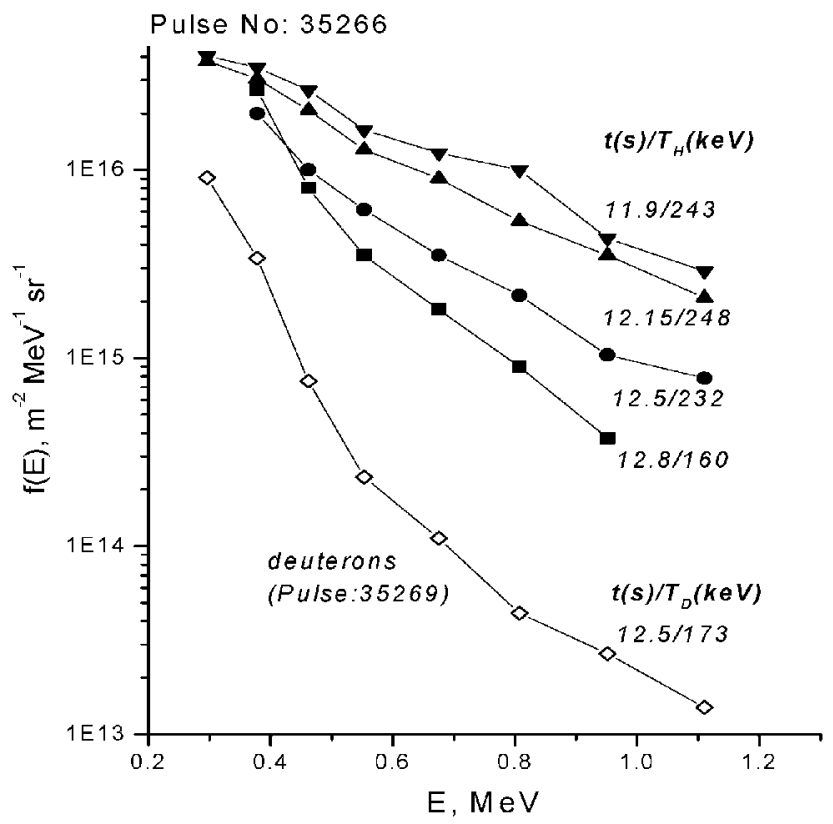

Fig. 23. Evolution of the ICRH-accelerated proton distribution function during combined ICRH and deuterium NBI. The distribution function of the second-harmonic ICRH-driven deuterons is also shown.

range of pitch angles $\leq 5 \times 10^{-3}$. Procedures for deduction of the local effective temperature and minority density in the case of an anisotropic distribution function of ICRH-accelerated ions from the measured $f(E)$ were developed in Ref. 61.

The NPA measurements of the energy distribution functions of ICRH-accelerated ions have resulted in corroboration of a linear increase of the perpendicular temperature of the minority ions with the ICRH power density, ${ }^{61}$ predicted by the Stix model ${ }^{8}$ Redistribution of the power between different species was observed in the course of combined heating scenarios considered for application in ITER. As an example, Fig. 23 demonstrates a reduction of the tail temperature of the proton distribution function after injection of the deuterium heating beam at $12 \mathrm{~s}$. Starting from that time, a high-energy tail appears on the energy distribution function of deuterons, implying channeling of the ICRH power from first-harmonic heating of proton minority to second-harmonic heating of high-energy beam deuterons. ${ }^{62}$

Much attention was paid to the preparation for measurements of fluxes of fusion products in the JET D-T campaign in 1997. This activity has led to the theoretical prediction that IIN of alpha particles would be capable of providing helium fluxes sufficient for the measurement of the alphas' energy spectrum at the core of ITER plasma. ${ }^{63}$ The IIN of alpha particles is based on the double CXS reactions with two-electron species of main intrinsic plasma impurities: 


$$
\mathrm{He}^{2+}+\mathrm{C}^{4+}, \mathrm{Be}^{2+}, \mathrm{He} \rightarrow \mathrm{He}+\mathrm{C}^{6+}, \mathrm{Be}^{4+}, \mathrm{He}^{2+} .
$$

For the first time, the predicted flux of the helium atoms in the energy range 0.3 to $1.1 \mathrm{MeV}$ was measured during ICRH $\mathrm{D}\left({ }^{3} \mathrm{He}\right)$ heating in JET (Ref. 8). To make such measurements possible, the NPA was enhanced with a spectrometric scintillation detector setup, which greatly increased the contrast between signal counts and neutron noise. Comparison of the measured distribution function with that calculated using the bounce-averaged diffusion operator in a time-dependent two-dimensional FokkerPlanck equation for ICRH validated the IIN of helium ions (Fig. 24). The importance of the [He] ions of beryllium and carbon in the creation of the high-energy tail of the measured helium flux and, hence, for alpha-particle diagnostics was established. The conclusion was made that for the anticipated fusion reactivity in JET D-T experiments, the measurement of neutralized alpha-particle flux would be feasible. The measurements of the distribution function of the slowing-down fusion alphaparticles in JET D-T plasmas were made in high-fusion reactivity hot-ion $\mathrm{H}$-mode pulses ${ }^{64}$ using only NBI heat-

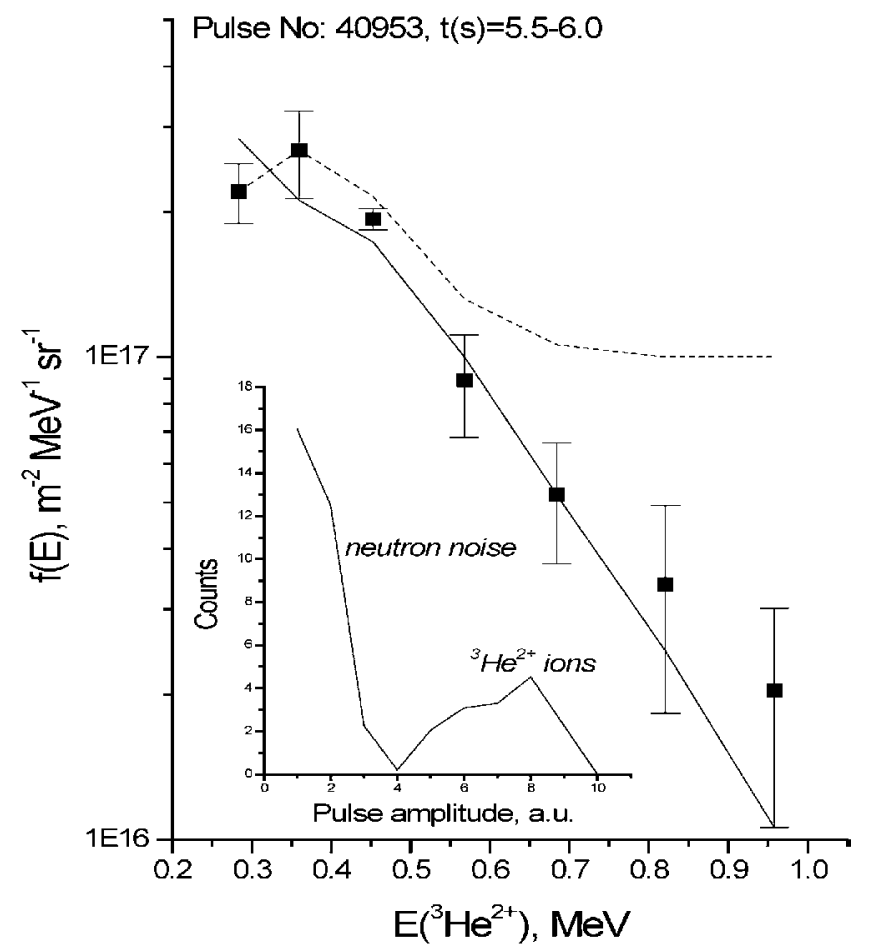

Fig. 24. Distribution function for ICRH-driven ${ }^{3} \mathrm{He}^{2+}$. The solid curve is for calculations using IIN modeling. The dashed curve was calculated ignoring the contribution of $\mathrm{Be}^{2+}$ and $\mathrm{C}^{4+}$ to the neutralization of ${ }^{3} \mathrm{He}^{2+}$ ions. The pulse-height spectrum of the detector output of the NPA channel set up for ${ }^{3} \mathrm{He}^{2+}$ at $680 \mathrm{keV}$ is also shown. The detection efficiency for neutrons is $10^{-5}$ of that for ions at the peak of ion distribution. ing, comprising $80-\mathrm{keV} \mathrm{D}$ atoms and $140-\mathrm{keV} \mathrm{T}$ atoms, giving a $\mathrm{D}-\mathrm{T}$ fuel mixture ${ }^{16} n_{\mathrm{D}} /\left(n_{\mathrm{D}}+n_{\mathrm{T}}\right) \sim 0.5$. The measurements were carried out with the NPA set up to detect ${ }^{4} \mathrm{He}$ atoms in eight channels in the energy range 0.8 to $3.0 \mathrm{MeV}$. The ${ }^{4} \mathrm{He}$ atoms were separated from all hydrogen isotope atoms except deuterium (having the same $Z / A$ ratio), making ${ }^{4} \mathrm{He}$ of energy $E_{\alpha}$ indistinguishable from $\mathrm{D}$ of energy $E_{d}=E_{\alpha} / 2$. For all pulses analyzed, a flux of neutral particles an order of magnitude higher than expected was observed in the low-energy channels. Most significantly, the low-energy flux arose long before alpha particles could have slowed down to the measurement energy. The analysis ${ }^{64}$ has shown that the excess flux should be attributed to high-energy deuterons produced by close elastic collisions (knock-on) between fusion alpha particles and thermal plasma fuel ions. ${ }^{65-67}$ Despite the low density of knock-on deuterons compared to that of alpha particles $\left[0.0025 \leq f_{d}(E) /\right.$ $\left.f_{\alpha}(\mathrm{E}) \leq 0.07\right]$, a flux of deuterium atoms to the lowenergy NPA channels exceeding the helium flux was produced because of the much higher neutralization probability for the deuterons. The derived distribution functions were shown to be in satisfactory agreement with calculations ${ }^{64}$ using a time-dependent three-dimensional Fokker-Planck code FPP-3D, which incorporates neoclassical transport and classical slowing-down of fusion alpha particles and knock-on deuterons (Fig. 25). The investigation has also shown that a better strategy for determining alpha-particle behavior in future D-T experiments would be to measure knock-on tritons in the range $E_{t} \leq 1(\mathrm{MeV})$ rather than direct measurements of alpha particles or the knock-on deuterons. This is because the knock-on tritons will be well separated from the alpha particles in the NPA, unlike the deuterons, and the IIN of tritons is much more efficient than that for alpha particles.

\section{V.B. Information on the Confined Alpha-Particle Population in the Neutron Emission Spectrum}

Neutron emission spectrum (NES) measurements are used to provide information on the fusion reactivity in deuterium and D-T plasmas, including its detailed dependence on the velocity distribution of the fuel ion population. Thermal (Maxwellian) plasmas produce spectra of Gaussian shape whose width is determined by the Doppler broadening, the reflecting ion temperature. Deviation from the Gaussian shape signals the presence of suprathermal velocity components that appear in conjunction with ICRH and/or NBI, which also form the NES high-energy tail. High-energy tails can also arise from fusion products such as the alpha knock-on neutron $(\mathrm{AKN})$ emission from reactions involving suprathermal components in the fuel ion population accelerated by $\alpha+\mathrm{D}$ and $\alpha+\mathrm{T}$ close collisions, giving maximum energies up to just below $E_{\alpha}$. The AKN spectrum thus extends well beyond that of D-T neutron (DTN) emission even with ICRH and NBI applied. 


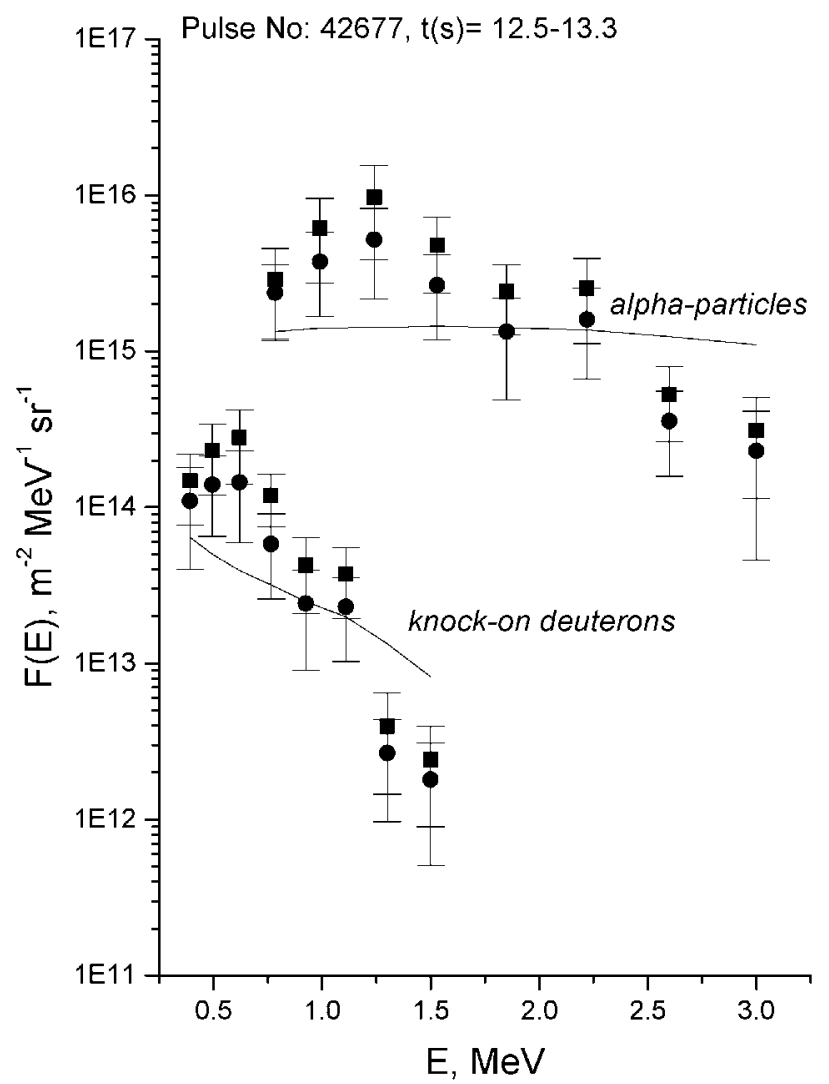

Fig. 25. Distribution functions of fusion alpha particles and knock-on deuterons ${ }^{64}$ deduced from the NPA measurements (points) and the modeling assuming classical confinement and slowing down (curves). The circles and squares show the results when the maximum and minimum values for neutralization probability are used.

The AKN effect was observed experimentally for the first time during the DTE1 campaign and was also theoretically modeled. ${ }^{68,69}$ The data were collected from 150 AKN events for all high-power NBI-heated plasmas with an integrated fusion power of $80 \mathrm{MWs}$ (Fig. 26). The AKN tail appears here at the last $20 \%$ of the measured spectrum (neutron energy range $E_{n}=15.7$ to $16.8 \mathrm{MeV}$ ), where it dominates over the DTN component. The observation is well described by a calculation with respect to the AKN/DTN ratio, assuming that the alpha-particle confinement and slowing down is classical. Moreover, the data consist of 10 discharges of different electron temperatures in the range 4 to $8 \mathrm{keV}$ whose neutron emission spectra were analyzed individually. The observed amplitude variation of the AKN/DTN is in line with the expected $T_{e}^{3}$ dependence due to the combined slowing down of the alpha particles and of the $\mathrm{D}$ and $\mathrm{T}$ fuel ions.

The most direct confined alpha-particle information from NES measurements concerns alpha-particle pressure $^{69}$; its variation over time could be probed by the

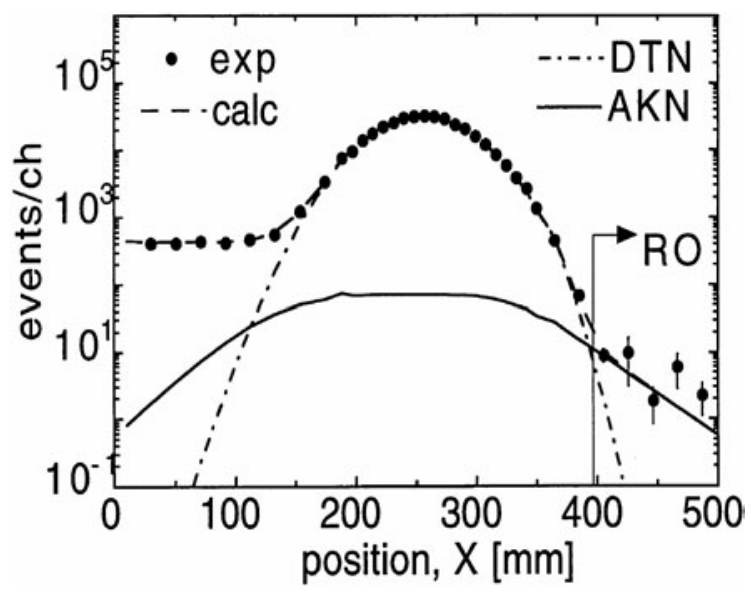

Fig. 26. Example ${ }^{68}$ of a recoil position histogram for the 14MeV NES measurements performed during the JET D-T discharge with NBI. The spectrum is decomposed into the contribution coming from deuterontriton neutron emission and the AKN emission based on model calculations. $\mathrm{RO}$ is the $\mathrm{AKN}$ region of observation of the full spectrum; the latter extends from $E_{n}=11$ to $17 \mathrm{MeV}$.

AKN/DTN ratio and quantitative information derived with the help of plasma model calculations. Moreover, NES measurements can provide information on the fast $\mathrm{D}, \mathrm{T}$, and alpha-particle populations and their relationship to the fusion reactivity. For quasi-steady state plasma conditions, this would provide empirical input to equilibrium calculations of the relationship between fusion reactivity (thermal and suprathermal components from absolute measurement of DTN) and its connection to the heating from the alpha-particle population (such as the birth-to-confinement ratio from the AKN/DTN ratio).

The measurements can be performed with high accuracy with known types of spectrometers, and the neutron flux from source to detection is practically unaffected by plasma and vessel windows. The data, therefore, have high reliability and a low level of systematic uncertainties, which benefits their use as benchmark input to plasma model calculations to extract embedded specific confined alpha-particle information. Here, NES is in a unique position as the provider of simultaneous information on both DTN and AKN reactivities for the separation and linkage of $P_{a u x}$ and $P_{\alpha}$ effects on burning plasmas, and this important technique developed on JET may be of significant interest for ITER (Ref. 1).

\section{V.C. Gamma-Ray Spectroscopy and Gamma-Ray Tomography in JET Plasmas with ICRH-Accelerated Ions}

On JET, the measurements of gamma-ray emission coming from nuclear reactions between energetic ions 

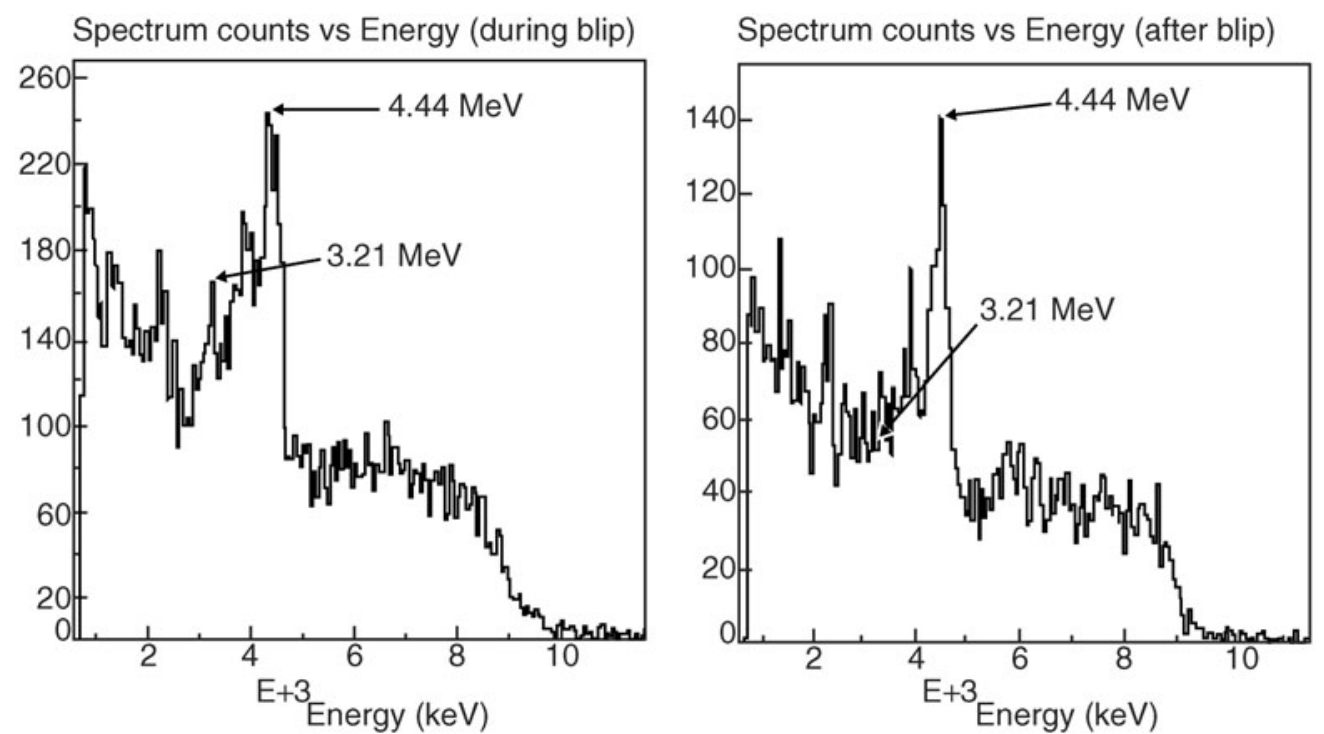

Fig. 27. Gamma-ray spectra measured ${ }^{80}$ in 2.0-MA, 2.25-T JET discharge (pulse 61046) with deuterium 15-MW NBI during 300 -ms blip of tritium NBI and just after the blip. Here, $P_{\mathrm{TNBI}} \approx 1.5 \mathrm{MW}, T_{e}(0) \approx 6 \mathrm{keV}$, and $n_{e}(0) \approx 6 \times 10^{19} \mathrm{~m}^{-3}$.

and impurity and fuel ions were first reported by Sadler et al. ${ }^{70}$ and have since been used to interpret a variety of fast ion effects during ICRH and NBI (Refs. 8, 11, and 71-73). There are three sources of fast particles that can give rise to gamma-ray emission from high-temperature plasmas. First, fusion reactions between the plasma fuel ions produce fusion products such as fast tritons (1.0 $\mathrm{MeV})$, protons $(3.0 \mathrm{MeV}),{ }^{3} \mathrm{He}(0.8 \mathrm{MeV})$, and ${ }^{4} \mathrm{He}(3.5$ and $3.7 \mathrm{MeV}$ ) ions. Second, ICRH of $\mathrm{H}$ and ${ }^{3} \mathrm{He}-$ minority ions accelerates these ions to energies in the $\mathrm{MeV}$ range. There are also ICRH schemes to accelerate $\mathrm{D}, \mathrm{T}$, and ${ }^{4} \mathrm{He}$ ions. Third, NBI heating introduces $\mathrm{D}, \mathrm{T}$, $\mathrm{H},{ }^{4} \mathrm{He}$, and/or ${ }^{3} \mathrm{He}$ ions. Fast ions born in the plasma produce line spectra due to their nuclear reactions with low- $Z$ plasma impurities. The fusion neutrons interact with the structural materials, generating a continuous $\gamma$-ray background.

A list of all essential nuclear reactions that have been identified in the gamma-ray spectra recorded in JET was given in Table 1 of Ref. 74. These reactions are classified by the types of fast ions interacting with different target ions in plasma. The table contains assessments of the minimum energy of the fast particles required to produce gamma-ray yields at levels that can be measured in JET. In particular, the table displays the reaction ${ }^{9} \mathrm{Be}(\alpha, n \gamma){ }^{12} \mathrm{C}$, the significance of which for the diagnosing helium ions in the $\mathrm{MeV}$ energy range was shown. ${ }^{11,75-77}$ This nuclear reaction is of a resonant type, which has thresholds. The presence of the $4.44-\mathrm{MeV}$ peak in the gamma-ray spectra is evidence for the existence of alphas with energies that exceed $1.7 \mathrm{MeV}$. The $3.21-\mathrm{MeV}$ gamma rays indicate that the alphas with energies in excess of $4 \mathrm{MeV}$ exist in the plasma. As an example,
Fig. 27 shows two gamma-ray spectra, recorded in the same JET discharge: The plot on the left shows the spectrum during a 300-ms blip of tritium NBI; the one on the right shows the spectrum just after this NBI blip. During the injection, two gamma-ray peaks, $4.44 \mathrm{MeV}$ and 3.21 $\mathrm{MeV}$, are observed; however, in the postblip time slice the 3.21-MeV peak becomes rather weak. This is an effect of changes in the distribution function, i.e., the result of a shift of the high-energy tail to the low-energy range due to the alpha particle slowing down. ${ }^{76}$

In alpha-particle simulation experiments with thirdharmonic heating of ${ }^{4} \mathrm{He}$ beam in ${ }^{4} \mathrm{He}$ plasmas, ${ }^{11,77}$ gamma radiation due to the nuclear reaction ${ }^{9} \mathrm{Be}\left({ }^{4} \mathrm{He}, n \gamma\right){ }^{12} \mathrm{C}$ was detected, showing the successful ICRH acceleration of ${ }^{4} \mathrm{He}$ beam ions as intended. The first energy level, 4.44 $\mathrm{MeV}$, of the final nucleus, ${ }^{12} \mathrm{C}$, is excited by alpha particles, and as a result the peak at $4.44 \mathrm{MeV}$ in the spectrum appears. The gamma-ray emission from the reaction ${ }^{12} \mathrm{C}(\mathrm{D}, p \gamma){ }^{13} \mathrm{C}$ was observed as well. A peak at 3.09 $\mathrm{MeV}$ (transition $3.09 \rightarrow 0$ ), which is identified as a gamma emission from the ${ }^{12} \mathrm{C}(\mathrm{D}, p \gamma){ }^{13} \mathrm{C}$ reaction, reflects the presence in the plasma of the fast $\mathrm{D}$ ions in the $\mathrm{MeV}$ range. This evidence indicates that the deuterium minority also absorbs some ICRH power at the third-harmonic D resonance that coincides with the third harmonic ${ }^{4} \mathrm{He}$ resonance. Figure 28 shows the gamma-ray spectra recorded during $1 \mathrm{~s}$ in two similar discharges with a highresolution $\mathrm{NaI}(\mathrm{Tl})$ spectrometer. It is seen that when the $110-\mathrm{keV}$ neutral beam heating injector was replaced by the $70-\mathrm{keV}$ one with the same power, the intensity of the 4.44-MeV gamma emission fell substantially, whereas the deuterium gamma peak did not change. This effect can be explained by decreasing single-pass ${ }^{4} \mathrm{He}$ damping 


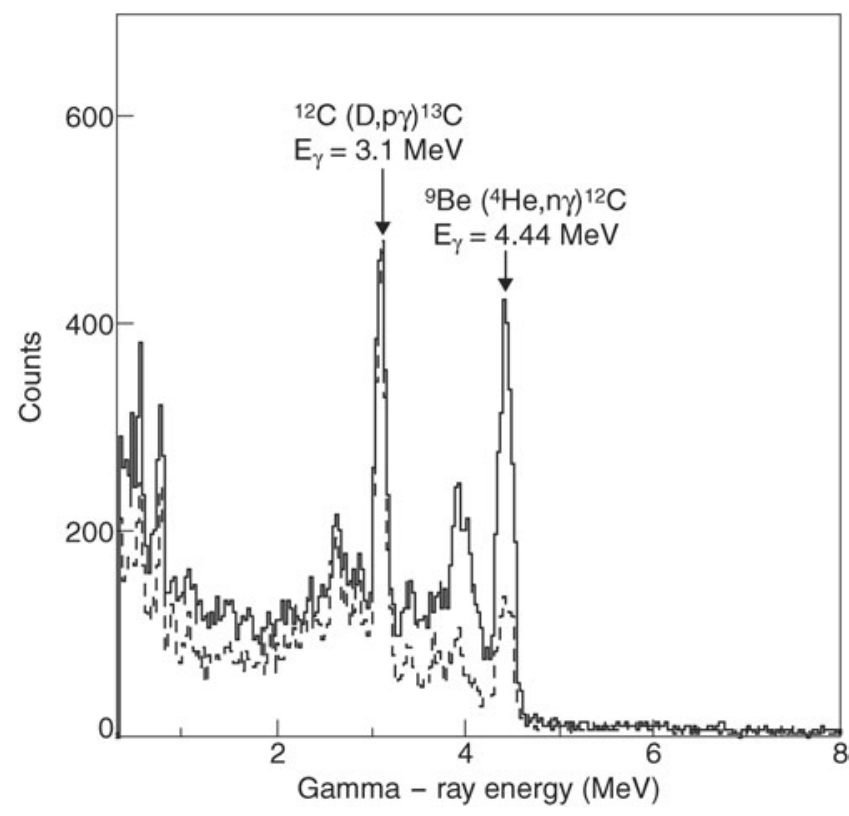

Fig. 28. Gamma-ray spectra measured ${ }^{77}$ by the $\mathrm{NaI}(\mathrm{Tl})$ detector. The solid line shows the spectrum recorded in a discharge with 70- and $110-\mathrm{keV}^{4} \mathrm{He} \mathrm{NBI}$; the dashed line shows the spectrum recorded in a discharge with two $70-\mathrm{keV}{ }^{4} \mathrm{He}$ NBI.

of the ICRH waves due to the smaller value of the finite Larmor radius of the fast ions that determines the third cyclotron harmonic absorption.

In JET gamma-ray energy spectra are measured with two different devices, a horizontal and a vertical line of sight through the plasma center. ${ }^{74}$ The first spectrometer is a calibrated bismuth germanate (BGO) scintillation detector with $75-\mathrm{mm}$ diameter and $75-\mathrm{mm}$ height that is located in a well-shielded bunker and views the plasma tangentially. To reduce the neutron flux and the gammaray background, the front collimator is filled with polythene. Behind the scintillation detector there is an additional dump of polythene and lead. The gamma rays are continuously recorded in all JET discharges over the energy range 1 to $28 \mathrm{MeV}$, with an energy resolution of about $4 \%$ at $10 \mathrm{MeV}$. The second device for gamma-ray energy spectrum measurements is a $\mathrm{NaI}(\mathrm{Tl})$ scintillation detector with a diameter of $125 \mathrm{~mm}$ and a height of $150 \mathrm{~mm}$ that views the plasma vertically through the center.

The spatial distribution of the gamma-ray emission sources in the JET plasma is measured using the neutron profile monitor shown in Fig. 29. The monitor consists of two cameras, vertical and horizontal, with 9 and 10 lines of sight, respectively. To measure the gamma-ray emission profile in the energy range $E_{\gamma}>1 \mathrm{MeV}$, we use the fast electrons-bremsstrahlung diagnostic system, which is incorporated into the neutron profile monitor. The detector array is composed of the $19 \mathrm{CsI}(\mathrm{Tl})$ photodiodes

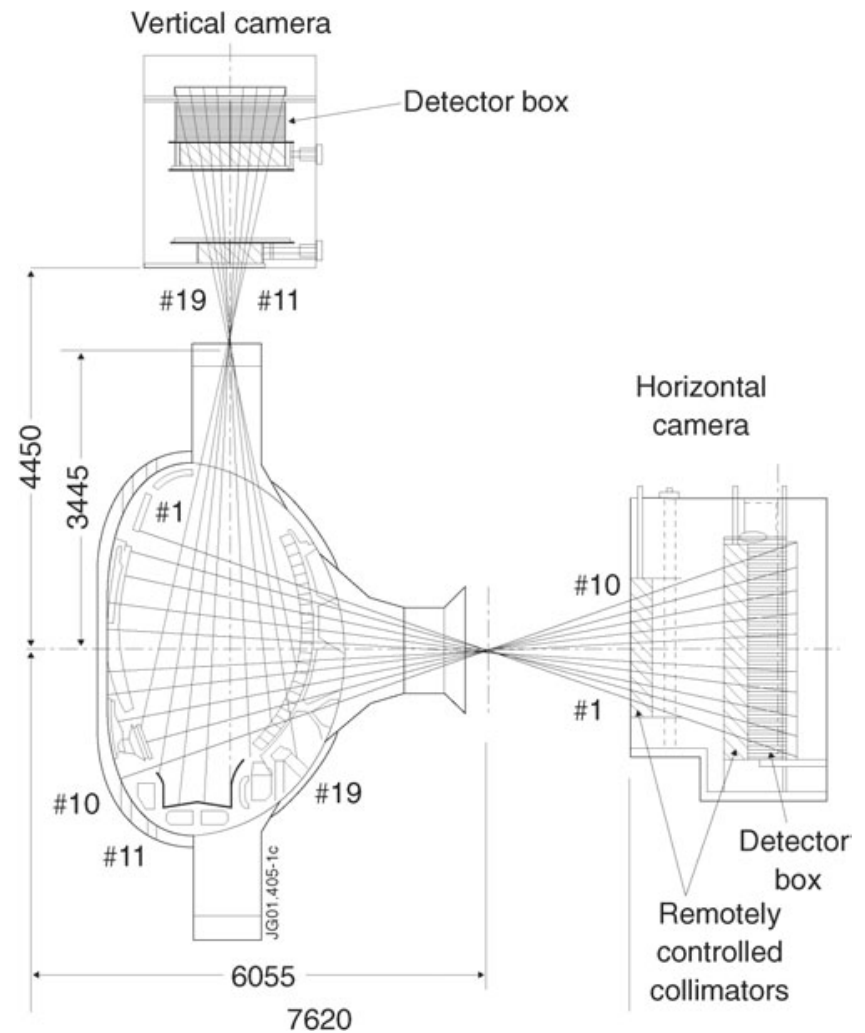

Fig. 29. Schematic view of the JET neutron emission profile monitor used for the spatial gamma ray emission measurements. $^{74}$

$(10 \mathrm{~mm} \times 10 \mathrm{~mm} \times 15 \mathrm{~mm})$. The photodiodes are remotely placed for measurements in the front of neutron detectors within each collimation channel. The data acquisition system accommodates the gamma-ray count rate measurement in four energy windows. This allows the allocation of specific gamma-ray peaks in the windows to be counted separately. A typical example of the tomographic reconstruction of the measured line-integrated profiles is shown in Fig. 30. It is seen clearly that the gamma-ray emission profile produced by fast $\mathrm{D}$ ions differs from the profile from ${ }^{4} \mathrm{He}$ ions accelerated by ICRH (Ref. 77). This effect can be explained by the initial difference in pitch angle distribution between ${ }^{4} \mathrm{He}$ beam ions injected into the plasma quasi-tangentially and isotropic D-minority ions.

To identify fast particles that exist in the plasma and give rise to the observed gamma-ray emission and to assess the effective tail temperatures of these fast ions, a dedicated code for the gamma-ray spectrum modeling, the GAMMOD code, has been developed based on the known nuclear reaction cross sections; it includes about a hundred gamma-ray transitions in the final nuclei of the nuclear reactions. ${ }^{74}$ Measurements from JET experiments in recent years indicate that the gamma-ray diagnostics work well, but further substantial improvement 


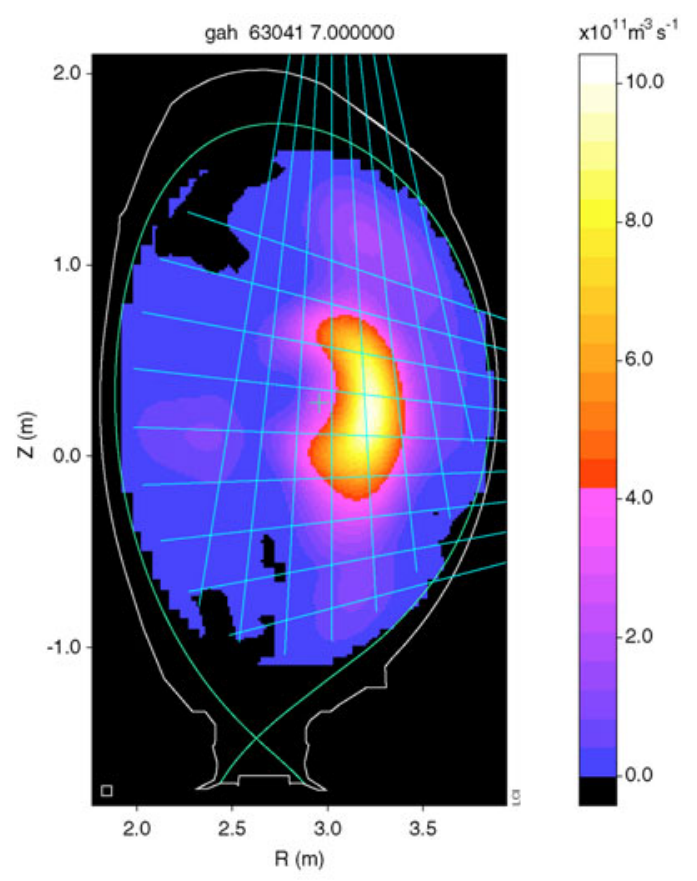

${ }^{4} \mathrm{He}$ in monotonic $q(r)$-plasma

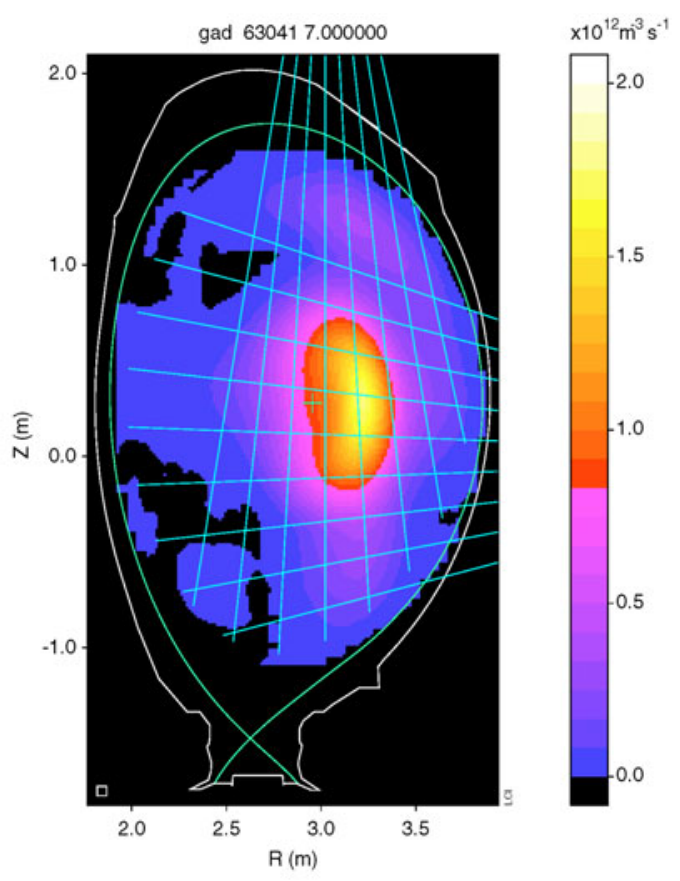

$D$ in monotonic $q(r)$-discharge

Fig. 30. Tomographic reconstructions of $4.44-\mathrm{MeV}$ gamma-ray emission from nuclear reaction ${ }^{9} \mathrm{Be}\left({ }^{4} \mathrm{He}, n \gamma\right){ }^{12} \mathrm{C}(\mathrm{left})$ and 3.09-MeV gamma-ray emission from the reaction ${ }^{12} \mathrm{C}(\mathrm{D}, p \gamma){ }^{13} \mathrm{C}$ (right) deduced from simultaneously measured profiles. ${ }^{77}$

of the diagnostics is proposed for measurements in highperformance D and D-T discharges to demonstrate the capabilities of gamma-ray diagnostics for burning plasma physics in future reactors. In particular, the development of gamma-ray spectrometers based on fast heavy scintillators and of a data acquisition system with fast digitizers $^{78}$ and the installation of the efficient neutron attenuators ${ }^{79}$ has been proposed. The main goals of this project are improving the energy and temporal resolution of the diagnostics and reducing the gamma-ray background.

\section{V.D. First Gamma-Ray Measurements of Fusion-Born Alpha Particles in Trace Tritium Experiments in JET}

The gamma-ray measurements ${ }^{80}$ of fusion-born alpha particles were done in JET "trace tritium" discharges, i.e., in majority deuterium plasmas after seeding with a small population of tritium NBI fast ions. The gammaray emission from the nuclear reaction ${ }^{9} \mathrm{Be}(\alpha, n \gamma){ }^{12} \mathrm{C}$ is used to measure changes in the density of the fast alpha particles with energy $E_{\alpha}>1.7 \mathrm{MeV}$ in the post-NBI period. This diagnostic nuclear reaction has already been applied to detect the presence of the fast alpha particles in JET experiments as simulated by ${ }^{4} \mathrm{He}$ beam ions accelerated with ICRH to the $\mathrm{MeV}$ range. ${ }^{11}$
In the experiments gamma-ray energy spectra are measured with a calibrated $\mathrm{BGO}$ scintillation detector ${ }^{74}$ with diameter of $75 \mathrm{~mm}$ and a height of $75 \mathrm{~mm}$. The detector is located in a well-shielded bunker and views the plasma quasi-tangentially. The detector line of sight lies in a horizontal plane about $30 \mathrm{~cm}$ below the plasma magnetic axis. During these experiments, the gamma rays were continuously recorded with integration time $250 \mathrm{~ms}$ over the energy range 1 to $28 \mathrm{MeV}$, with an energy resolution of about $4 \%$ at $10 \mathrm{MeV}$.

Diagnostic capabilities of the nuclear reaction ${ }^{9} \mathrm{Be}(\alpha, n \gamma){ }^{12} \mathrm{C}$ are determined by the specific reaction cross section. The resonance structures in the excitation functions of the first two levels of the final nucleus, ${ }^{12} \mathrm{C}$, populated in this reaction provide the energy selectivity for the alpha-particle measurements. The first energy level, 4.44 MeV, is excited by alpha particles with energies exceeding $1.7 \mathrm{MeV}$, and the second one, $7.65 \mathrm{MeV}$, is populated by alpha particles with energies in excess of 4 $\mathrm{MeV}$. The beam-plasma alpha particles can give rise to 4.44-MeV gammas $(4.44 \rightarrow 0$ transition) and can also excite the second level, giving rise to gamma rays with energy $3.21 \mathrm{MeV}(7.65 \rightarrow 4.44$ transition).

Clear variations in the intensity of the $4.44-\mathrm{MeV}$ gamma-ray emission were observed in the post-beam blip period of many discharges. Figure 31 shows decays of the $4.44-\mathrm{MeV}$ gamma-ray intensity, recorded by the 


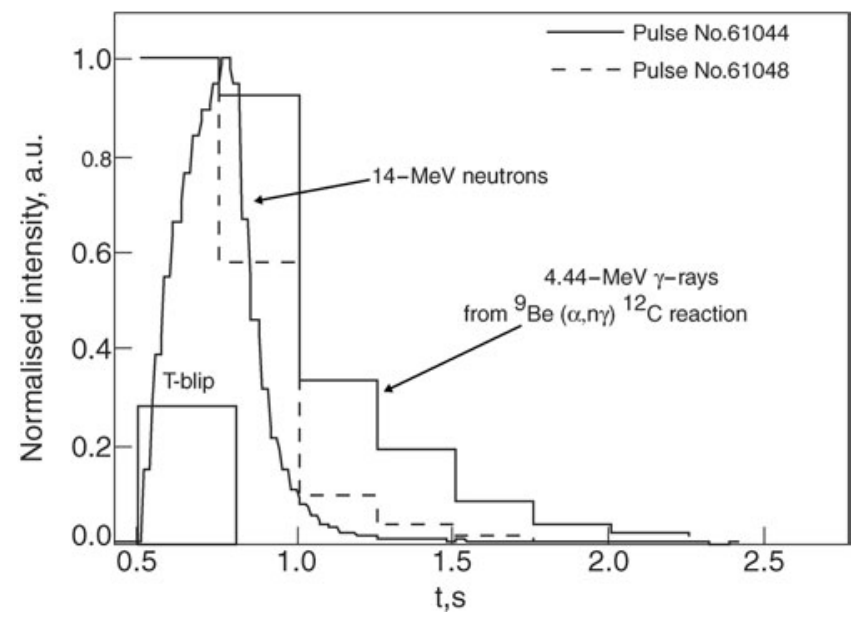

Fig. 31. Comparison ${ }^{80}$ of time evolutions of 4.44-MeV $\gamma$-ray emission measured in discharges 61044 and 61048 . Pulse 61044: $2.0 \mathrm{MA}, 2.25 \mathrm{~T} ; P_{\mathrm{DNBI}} \cong 14.5 \mathrm{MW}$, $P_{\mathrm{TNBI}} \cong 1.5 \mathrm{MW} ; T_{e}(0) \cong 5 \mathrm{keV}, n_{e}(0) \cong 4.8 \times$ $10^{19} \mathrm{~m}^{-3}$. Pulse 61048: $2.0 \mathrm{MA}, 2.25 \mathrm{~T} ; P_{\mathrm{DNBI}} \cong$ $2.9 \mathrm{MW}, P_{\mathrm{TNBI}} \cong 2.3 \mathrm{MW} ; T_{e}(0) \cong 3.5 \mathrm{keV}, n_{e}(0) \cong$ $3.2 \times 10^{19} \mathrm{~m}^{-3}$.

spectrometer in discharges with different NBI heating powers. The measured rate of $14-\mathrm{MeV}$ neutrons, which are born during the T-beam injection, is shown as well. The decays of the gamma-ray emission are measured against unchanging plasma conditions. In these experiments the duration of T-beam blips was $t_{\text {blip }} \leq 300 \mathrm{~ms}$.

More than 20 discharges were analyzed. Correlation between the $\tau_{\gamma}$ decay time of the $4.44-\mathrm{MeV} \gamma$-ray intensity from the reaction ${ }^{9} \mathrm{Be}(\alpha, n \gamma){ }^{12} \mathrm{C}$ and the plasma parameters in different plasma scenarios was established. The interpretation of the experimentally observed evolution of the alpha-produced gamma-ray emission requires not only a simulation of the alpha-particle transport but an equally accurate description of the time dependence and the phase space shape of the fusion source determined by the tritium NBI blips and the deuterium plasma. Due to the essential contribution of beam-target and beam-beam fusions to alpha production, the source term shape in spatial and velocity coordinates is determined by the tritium behavior as well as by the distribution function of deuterium.

\section{V.E. Interferometry/Reflectometry Detection of Fast Ion-Driven Alfvén Cascade Eigenmodes in Reversed Shear JET Discharges}

The first O-mode interferometry-like measurements of AEs driven by energetic ions accelerated by ICRH in JET were performed in 2003 (Ref. 18). This technique, which measures line-integrated electron density perturbations, has been used to detect Alfvén cascade (AC) eigenmodes ${ }^{19,83}$ typical of reversed-shear advanced to- kamak scenarios in JET. It shows an unprecedented frequency and time resolution of the eigenmodes, far superior to those obtained by external magnetic coils or by the ECE radiometer reported earlier. ${ }^{19,81}$

This diagnostic probes the midplane plasma from the outer side of the torus (at major radius $R \approx 4 \mathrm{~m}$ ) and toward the high magnetic field region along a radial line of sight. Initially applied to density profile measurements, ${ }^{82}$ this diagnostic tool now works with up to 10 fixed-frequency channels for density perturbation measurement. The microwave beam propagating through the plasma undergoes a change in amplitude and a shift in phase proportional to the integration of the refractive index $N(R)=\left[1-n_{e}(R) / n_{e}^{\text {crit }}\right]^{1 / 2}$ along the line of sight. Here, the plasma electron density is the sum of the equilibrium density $n_{0}(R)$ and the perturbed density $\delta n(R)$ caused by AEs, $n_{e}(R)=n_{0}(R)+\delta n(R)$, and $n_{e}^{\text {crit }}$ is the critical density determined for microwaves with frequency $\omega=\omega_{p e}$, where $\omega_{p e}$ is the plasma frequency. The density perturbations $\delta n$ induce perturbations of both the amplitude $A$ and the phase $\phi$ of the probing microwave beams by comparing the beam propagating through the plasma with the reference beam outside the plasma. The beam propagating through the plasma is reflected from the inner wall of the torus (at $R \approx 2 \mathrm{~m}$ ) in the interferometry regime and from the plasma in the reflectometry regime. The receiver measuring the returned beam is connected to a digital converter with a sampling rate of $1 \mathrm{MHz}$, and the data for $A \cos \phi$ and $A \sin \phi$ are recorded with 14-bit effective resolution for $3 \mathrm{~s}$ during discharges.

Figure $32 \mathrm{a}$ shows time traces of the input power and the plasma parameters from a $B_{T} \approx 2.75 \mathrm{~T}, I_{P} \approx 1.7 \mathrm{MA}$ JET discharge, in which lower hybrid current drive (LHCD) was applied during the current ramp-up phase to obtain a reversed-shear magnetic configuration. ICRH power was used for accelerating hydrogen minority ions to energies high enough for the ions to resonate with shear Alfvén waves. Figure 32b shows the fixed frequencies of the six-channel microwave beams used in the experiment versus the cutoff frequency determined by the plasma density profile. These probing beams operate in the interferometry regime if the maximum plasma density is below $0.43,1.06,1.44,1.94,2.54$, or 3.16 $\left(\times 10^{19} \mathrm{~m}^{-3}\right)$, respectively, for the six beams. Figure 33 shows both the interferometry and the external magnetic coil measurements of Alfvén modes excited by the fast ions in this discharge. The Alfvén frequency spectrum consists of many frequency-sweeping discrete modes observed in the frequency band from $40 \mathrm{kHz}$ to the TAE frequency range, $140 \mathrm{kHz}$, in agreement with the wellestablished characteristics of the ACs (Refs. 19, 81, and 83 ). Figure 33 a shows the interferometry measurements of the density perturbation associated with AC modes, indicating that very high resolution in both time and frequency with high sensitivity can be achieved. The high sensitivity of this measurement technique allows the observation of ACs with toroidal mode numbers up to 

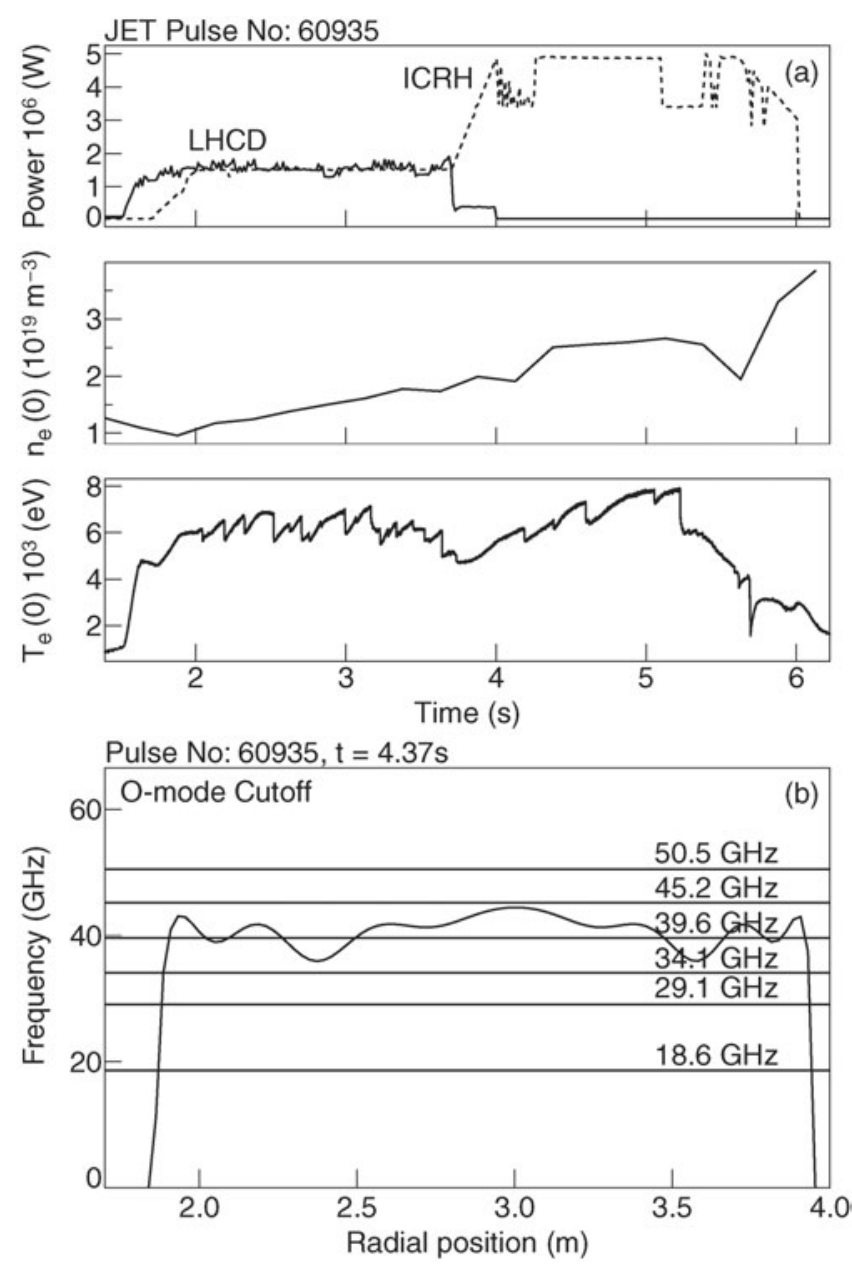

Fig. 32. Discharge 60935 in JET (Ref. 18). (a) Time traces of LHCD and ICRH power, on-axis electron density, and central electron temperature. (b) Frequency of the $\mathrm{O}$-mode cutoff as a function of major radius calculated from the measured electron density profile together with frequencies of the six microwave beams launched from $R \approx 4 \mathrm{~m}$.

$n=16$ (inferred from the slope $\left.d \omega_{A C} / d t\right)$. In comparison, the magnetic probe data shown in Fig. 33b detected ACs with mode numbers only up to $n=5$ and during a much shorter time window. The physics interpretation of the data is independent of the choice between the amplitude $A$ and the phase shift $\phi$ of the reflected signal since they both show the ACs. However, the clearest images of the ACs were obtained on spectrograms of the amplitude $A$ and of the homodyne signals $A \cos \phi$ or $A \sin \phi$ due to the way the signals are recorded in JET. In accordance with Ref. 19, an AC starts to exist if the condition $m-n q_{\min }(t)=0$ becomes valid, where both $m$ and $n$ are integers. It follows, then, that for a $q_{\text {min }}(t)$, which decreases in time, ACs of different mode numbers $m, n$ are excited one by one, scanning the condition $m / n=q_{\min }(t)$ in time. Figure 33a, then, is showing the sequence of ACs

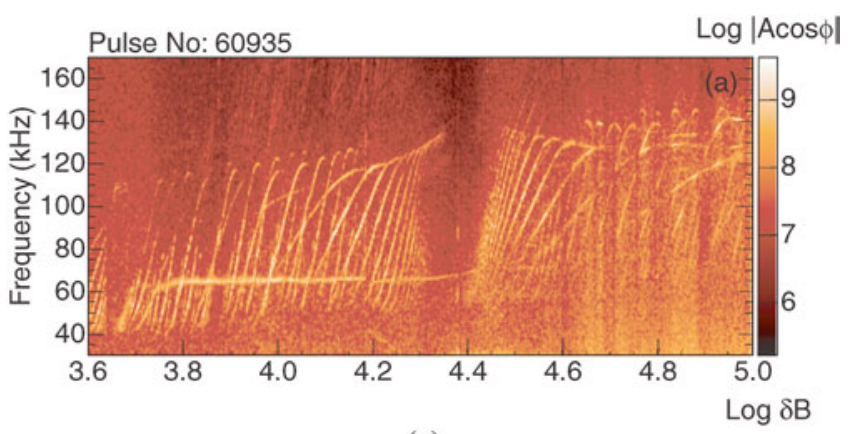

(a)

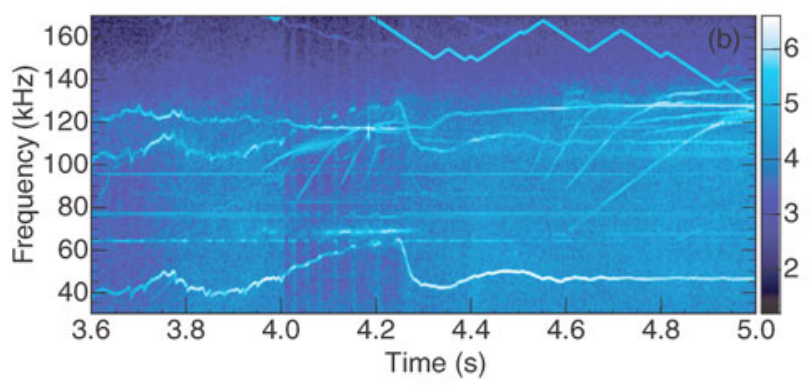

(b)

Fig. 33. Fourier spectrograms ${ }^{18}$ showing ACs with different toroidal and poloidal mode numbers in the discharge shown in Fig. 32. (a) Interferometry measurements with microwave beam of $45.2 \mathrm{GHz}$. (b) Measurements with external magnetic pickup coil.

associated with all possible (low) rational values through which $q_{\min }(t)$ passes. Therefore, monitoring of the value of $q_{\min }(t)$ with a very high accuracy, up to $\Delta q_{\min } \approx \frac{1}{16}$, can be performed based on the interferometry measurements. The appearance times of the ACs also can be used as a diagnostic of rational magnetic surfaces passing through a layer at $r \approx r_{\min }$ associated with the magnetic surface at $q_{\min }(t)$. In particular, Fig. 33a shows clearly that at $t \approx 4.4 \mathrm{~s} \mathrm{ACs}$ of all possible mode numbers are excited simultaneously, indicating the appearance of $q_{\min }(t)=$ integer. ${ }^{19,81}$ A clear gap in the density of the ACs observed just before this time shows the depletion of the rational $m / n$ values, as discussed in Ref. 84 .

An important correlation in time is observed in JET (Ref. 85) between the ITB-triggering events and "grand" ACs marking the appearance of $q_{\min }(t)=$ integer magnetic surfaces. Often, the ITB-triggering events precede the observation of grand ACs. This correlation is observed for a variety of JET reversed-shear discharges with plasma densities up to $5 \times 10^{19} \mathrm{~m}^{-3}$ and for various magnetic fields and plasma currents. ${ }^{86}$ Use of this correlation allows a better development of new advanced scenarios in JET.

In summary, a new interferometry technique was developed for monitoring the discrete spectrum of Alfvén cascade eigenmodes driven by ICRH-accelerated ions in "advanced" scenarios of the JET tokamak. Similar 
techniques based on higher probing frequency microwaves may be applied for diagnosing plasma of ITERtype tokamak-reactors.

\section{V.F. Simultaneous Time-Resolved Measurements of ICRH-Accelerated ${ }^{3} \mathrm{He}$ lons with $E>500 \mathrm{keV}$ and Coupled Energetic Ion-Driven AEs}

To study coupling between fast ions and AEs in the regime of small drift orbits of fast ions, $\Delta_{\text {drift }} / a \approx 0.1 \ll$ 1, relevant for ITER-type machines, JET developed a scenario for AEs excited with ICRF-accelerated ${ }^{3} \mathrm{He}$ minority in ${ }^{4} \mathrm{He}$ plasma ${ }^{87}$ Fast ${ }^{3} \mathrm{He}$ ions with energy as low as $500 \mathrm{keV}$ can interact with $\mathrm{C}$ and $\mathrm{Be}$ impurities and generate gamma rays through nuclear reactions much more numerous ${ }^{74}$ than those with ${ }^{4} \mathrm{He}$. Due to the high intensity of this gamma-ray emission from ${ }^{3} \mathrm{He}$, timeresolved measurements of fast ${ }^{3} \mathrm{He}$ become possible. The choice of helium plasma instead of deuterium was determined for two main reasons. First, for similar ion temperatures, ${ }^{4} \mathrm{He}$ thermal ions have lower speed than deuterium thermal ions, so the ion Landau damping of AEs due to the $V_{\| i}=V_{A} / 3$ resonance is exponentially smaller for AEs in ${ }^{4} \mathrm{He}$ plasma. Second, the low level of neutrons makes the gamma-ray measurements nearly noise free, so a better-quality gamma-ray image of the fast ${ }^{3} \mathrm{He}$ ions, with time resolution up to $10 \mathrm{~ms}$, could be obtained.

Figure 34 shows simultaneous measurements of the $\mathrm{AE}$ activity in the frequency range up to $450 \mathrm{kHz}$ with Mirnov coils, together with the intensity of gamma-ray

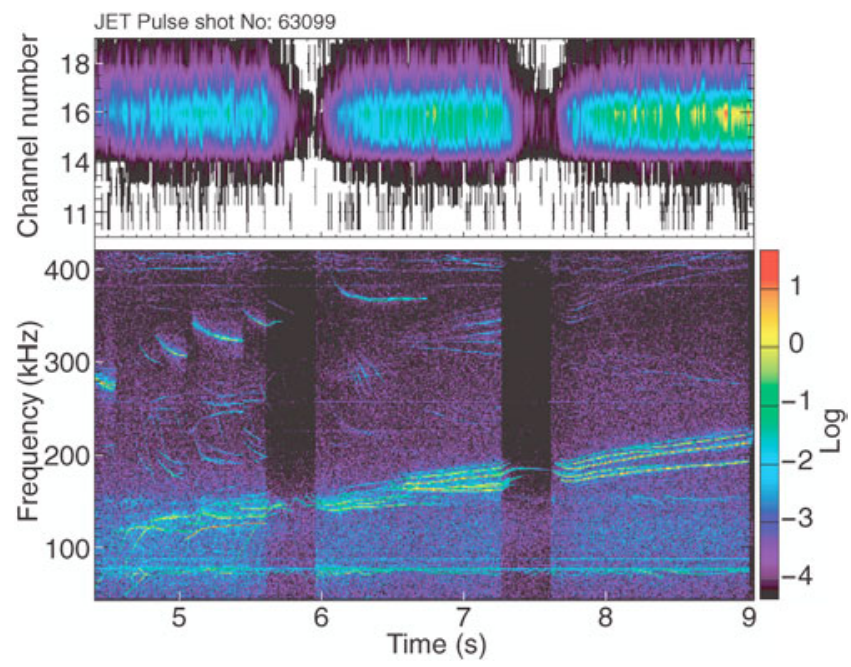

Fig. 34. Top: Intensity of $\gamma$ rays born in nuclear reactions between fast ${ }^{3} \mathrm{He}$ and Be as measured with vertical $\gamma$-ray camera (channel 11 is at the inner side of the torus and channel 19 at the outer side, as Fig. 29 shows). Bottom: Magnetic spectrogram showing ACs, TAEs, and EAEs excited by fast ${ }^{3} \mathrm{He}$ ions at the time of measurements of ${ }^{3} \mathrm{He}$ profile discharge 63099 in JET (Ref. 87). emission measured by the vertical gamma-ray camera in JET helium discharge 63099. In this discharge, the magnetic field and maximum plasma current were $B=3.3 \mathrm{~T}$ and $I=2.3 \mathrm{MA}$, respectively, the electron temperature and density were $T_{e}(0) \approx 6.5 \mathrm{keV}$ and $n_{e}(0) \approx 2.5 \times$ $10^{19} \mathrm{~m}^{-3}$, and the plasma composition was ${ }^{4} \mathrm{He}: \mathrm{D}=$ 91\%:9\%. LHCD power of $1.7 \mathrm{MW}$ was applied during the current ramp-up phase to obtain a reversed shear magnetic configuration, and ICRH power of $5 \mathrm{MW}$ was applied for the on-axis heating of ${ }^{3} \mathrm{He}$ minority ions. Notches in the ICRH power from $5 \mathrm{MW}$ down to $1 \mathrm{MW}$ were performed in this discharge at time slices $5.6 \mathrm{~s}$ and $7.2 \mathrm{~s}$ in order to observe both the decay and increase of the ${ }^{3} \mathrm{He}$ fast ion population with $E>500 \mathrm{keV}$ on the gamma-ray diagnostics. It is seen in Fig. 34 that numerous AEs of different types were excited in this discharge: ACs (Ref. 81), TAEs (Ref. 27), and EAEs (Ref. 28). The notches in ICRH power affect significantly both the gamma ray emission from ${ }^{3} \mathrm{He}$ ions and the AEs, but different AEs are affected somewhat differently. Figure 34 shows that a sharp decrease in intensity of gamma ray emission from ${ }^{3} \mathrm{He}$ with $E>500 \mathrm{keV}$ is well correlated with the disappearance of the TAE modes. However, the ACs with frequencies approaching the TAE persist during the time of the notch in ICRH power, when no ${ }^{3} \mathrm{He}$ ions are detected with the gamma-ray camera. This indicates that the $\mathrm{AC}$ instability is caused by ${ }^{3} \mathrm{He}$ ions with energies lower than $500 \mathrm{keV}$, in correspondence with the observation of ACs in JET and on DIII-D driven with sub-Alfvénic NBI (Ref. 86). In spite of the high-amplitude AE activity in the whole frequency range covered by the MHD diagnostics, the time-resolved measurements of ${ }^{3} \mathrm{He}$ with $E>500 \mathrm{keV}$ detected no notable degradation of gamma-ray emission due to any of the AEs observed in these discharges with the orbit widths $\Delta_{\text {drift }} / a \ll 1$.

\section{V.G. Further Improvements of Fast Ion Diagnostics in JET}

Gamma-ray spectroscopy is now the most established technique to measure both the energy and the spatial distribution of the alpha particles in JET. The main limitation of this approach is due to the energy threshold of the nuclear reactions, which are the basis of the gamma ray emission. The reactions most used in JET have cross sections that fall off with energy quite rapidly for energies lower than about $1 \mathrm{MeV}$. This method is therefore very useful to detect the source and the high energy range of the alpha or fast particles. On the other hand, the energy range of more interest from the point of view of wave-particle interactions is below $1 \mathrm{MeV}$. To address this limitation of the JET diagnostic capability, a different approach based on the atomic physics of impurity ions is presently being developed. It consists of injecting an extrinsic high-Z impurity into the plasma. The energy levels of its highly ionized species found in the plasma 
core can be populated by collisions with energetic particles, such as the alphas, as well as electrons. Spectral line intensity ratios can therefore allow the energetic particle density to be determined. Preliminary experiments in which F-like $\mathrm{Kr}$ has been excited by fast $\mathrm{D}$ neutral beam particles have given positive results. The quantum mechanical calculations of the relevant cross sections are being finalized for F-like $\mathrm{Kr}$. By using transitions from different ions and other elements, this technique has the potential to give information on the velocity distribution functions of the alpha particles.

Another JET diagnostic capability, which lies in the field of lost alpha detection, is available now. A series of Faraday cups were installed during the 2004 and 2005 shutdown. ${ }^{88}$ The Faraday cups consist of an array of 13 individual detectors, each with a minimum of four $75 \mathrm{~mm} \times$ $25 \mathrm{~mm} \times 2.5 / 1 \mu \mathrm{m}$ Ni foils separated by $2.5 \mu \mathrm{m}$ phlogophite mica electrical insulating foils. Each detector allows a modest degree of energy resolution (between 20 and $50 \%$ depending on the detailed foil and aperture geometry), a time resolution of about $1 \mathrm{~ms}$, and a minimum detectable signal of about $0.5 \mathrm{nA}$. The detectors are distributed between three radial locations (equally spaced between 25 and $85 \mathrm{~mm}$ behind the adjacent poloidal limiter) at five poloidal locations between midplane and $80 \mathrm{~cm}$ below midplane. The heat load from the alpha-particle flux is dissipated by having the aperture in front of the foils consist of an array of 3-mm-diameter circular holes. The estimated temperature of the foils will consequently remain less than about $900 \mathrm{~K}$. The minimum detectable signal level is about $0.5 \mathrm{nA}$. A total of about 40 signals will therefore be available. The detectors have an energy resolution between 30 and $50 \%$ and a bandwidth of $1 \mathrm{kHz}$. This diagnostic could be implemented in ITER since it is rather robust and compatible with the ITER environment.

The Faraday cups are complemented by a scintillator probe located just below the equatorial plane. ${ }^{89}$ The scintillator device consists of a thin layer of P56 scintillator (0.63 lumens/W). The scintillator image is transmitted through a series of lenses and fiber optic bundles to a charge-coupled device and a photomultiplier tube outside the vessel with respective time resolutions of 20 and $3 \mathrm{~ms}$. The device will allow a pitch angle resolution of $5 \%$, a best achievable resolution of the gyroradius of $15 \%$, and a minimum detectable signal level of about $5 \times$ $10^{4} \alpha / \mathrm{s}$. To maintain a scintillator temperature below about $400^{\circ} \mathrm{C}$, an externally supplied water cooling system has been incorporated in the probe design.

The JET diagnostic was designed mainly with the detection of alpha particles born in fusion reactions $\left({ }^{4} \mathrm{He}\right.$, $3.5 \mathrm{MeV}$ ) in mind but is sufficiently flexible to also see high-energy hydrogen and deuterium. ${ }^{90}$ Its range of accessible pitch angles extends from 35 to 85 deg (5\% resolution); detectable energies depend on the particle type since only particles with gyroradii between 30 and $140 \mathrm{~mm}$ (15\% resolution) pass the collimator. It is equipped with P56 as the scintillating material, which shows high photon efficiency per ion and a moderately fast decay time ( $3 \mathrm{~ms}$ ) and is compatible with the environment in JET (hot vessel, neutron fluxes).

\section{CONCLUSIONS AND OUTLOOK}

One can now assess the JET results described in this chapter in the wider context of the present-day magnetic nuclear fusion research and the next-step burning plasma experiment. Analysis of electron heating by fusion-born alpha particles in JET D-T discharges has shown that the energy transfer from alpha particles to electrons is consistent with classical expectations and reveals no anomalous effects. In the same experiments, thermal ion heating was found to be higher than expected, possibly due to ion temperature internal barrier formation in D-T plasmas or other effects unaccounted for so far. Further investigation of thermal ion heating by fusion-born alpha particles is of significant interest for possible future D-T experiments. It is important to note, however, that the excessive value of thermal ion heating is a positive effect enhancing the fusion reactivity of the plasma. In summary, the heating study performed on JET did not reveal any anomalous effects that may prevent achievement of predicted fusion power in a next-step device.

No AEs driven by alpha particles were observed in the highest fusion power JET D-T hot-ion H-mode discharges. The presence and absence of AEs excited by ICRH-accelerated ions and alpha particles are consistent with the existing CASTOR-K model. AEs possibly driven by alpha particles together with ICRH-accelerated ions were observed in optimized shear JET plasmas with elevated $q$ profile in the afterglow phase after abrupt switching off of NBI and ICRH power. A study of alphaparticle-driven AEs in such scenarios will require development of a reproducible scenario for optimized shear plasmas with NBI only (no ICRH). This development also may be considered as an aim for possible future D-T experiments.

Experiments using an active antenna system to drive and detect modes in the Alfvén frequency range have shown the existence of weakly damped, stable eigenmodes, clearly identified as AEs of TAE and EAE frequency ranges. No other resonant modes were found in the plasma spectrum in the Alfvén wave frequency range $(20$ to $500 \mathrm{kHz})$. Eigenfrequencies of the stable AEs were found to be in good agreement with existing theory of AEs, and this allowed development of an MHD spectroscopy technique. In addition to the information on $\mathrm{AE}$ frequency, a large database of the $\mathrm{AE}$ damping rates has been accumulated in a variety of plasma configurations. This database is of interest for assessing the linear stability limits of AEs under different conditions. Following the successful active antenna experiment in JET, similar 
diagnostics have been installed on C-MOD and MAST, thus significantly expanding the $\mathrm{AE}$ spectrum database for high-density high-field plasmas (C-MOD) and highbeta plasmas (MAST).

Knock-on deuterons of $\mathrm{MeV}$ energy range born in close elastic collisions with alpha particles were detected for the first time in JET D-T experiments by two independent diagnostics, NPA and neutron emission spectroscopy. These measurements have demonstrated the possibility of obtaining information about alpha particles through knock-on deuterons, which are much easier to measure.

Diagnosis of energetic ions based on measuring gamma rays born in nuclear reactions between the energetic ions and fuel and impurity ions was favorably demonstrated in JET experiments. For the first time, this diagnostic technique measured simultaneously spatial profiles of two different energetic ion populations, D and ${ }^{4} \mathrm{He}$. It was also demonstrated for the first time that such diagnostics are capable of measuring gamma-ray emission from fusion-born alpha particles in tritium trace experiments. Measurement of gamma-ray profiles of ICRHaccelerated ${ }^{3} \mathrm{He}$ ions with high time resolution performed simultaneously with measurements of unstable AEs in a broad frequency range has demonstrated the possibility of assessing the properties of energetic ions coupled to significant AE activity. Further development of the gamma diagnostics will aim at the possibility of employing these measurements in high-power D-T plasmas by using neutron filters in the collimators and testing detectors, which would be sensitive to gamma rays but transparent to neutrons.

AEs remain one of the most important uncertainties in predicting alpha-particle behavior in future burning plasma devices. It is therefore crucial to develop diagnostic techniques that are able to unambiguously detect unstable AEs in plasmas. A new O-mode interferometry technique detecting electron density perturbations has shown for the first time the possibility to detect AEs with higher time and frequency resolution and higher sensitivity than Mirnov coils. Following this development, far-infrared interferometry and phase contrast imaging diagnostic techniques were employed for detecting highfrequency AEs in JET and worldwide.

A major enhancement of fast ion diagnostics in JET has been recently performed. This includes scintillator and Faraday cups for measuring fast ion losses, neutron spectrometer TOFOR, and dedicated active TAE antenna. All the new diagnostics have started to operate very successfully, and they significantly enhance JET as a machine for burning plasma studies.

\section{ACKNOWLEDGMENTS}

The authors thank B. Alper, S. Hacquin, M. Mantsinen, and V. Yavorskij for their valuable comments and C. Gorme- zano for important discussions and help in preparing this chapter. This work has been conducted under the European Fusion Development Agreement. The work of UKAEA personnel was partly funded by the U.K. Engineering and Physical Sciences Research Council and by Euratom. A. Fasoli and D. Testa were partly supported by the Fond National Suisse pour la Recherche Scientifique, grant 620-062924. The views and opinions expressed herein do not necessarily reflect those of the European Commission.

\section{REFERENCES}

1. ITER Physics Basis, Chap. 5, Nucl. Fusion, 39, 2471 (1999).

2. P. H. REBUT and B. E. KEEN, Fusion Technol., 11, 13 (1987).

3. The JET Project, Scientific and Technical Developments in 1976, p. 35, Commission of the European Communities Directorate General (1977).

4. G. J. SADLER et al., Plasma Phys. Control. Fusion, 34, 1971 (1992).

5. G. A. COTTREL and D. F. H. START, Nucl. Fusion, 31, 61 (1991).

6. D. F. H. START et al., Nucl. Fusion, 39, 321 (1999).

7. L.-G. ERIKSSON et al., Nucl. Fusion, 39, 337 (1999).

8. J.-M. NOTERDAEME et al., "Physics Studies with the Additional Heating Systems in JET," Fusion Sci. Technol., 53, 1103 (2008).

9. S. E. SHARAPOV et al., Nucl. Fusion, 39, 373 (1999).

10. M. KEILHACKER et al., Nucl. Fusion, 39, 209 (1999).

11. M. J. MANTSINEN et al., Phys. Rev. Lett., 88, 105002 (2002).

12. G. DUESLING et al., Fusion Technol., 11, 163 (1987).

13. J. JACQUINOT, G. J. SADLER, and the JET TEAM, Fusion Technol., 21, 2254 (1992).

14. T. H. STIX, Plasma Phys., 14, 367 (1972).

15. G. TAYLOR et al., Phys. Rev. Lett., 76, 2722 (1996).

16. P. R. THOMAS et al., Phys. Rev. Lett., 80, 5548 (1998).

17. A. FASOLI et al., Phys. Plasmas, 7, 1816 (2000).

18. S. E. SHARAPOV et al., Phys. Rev. Lett., 93, 165001 (2004).

19. S. E. SHARAPOV et al., Phys. Lett. A, 289, 127 (2001).

20. C. GORMEZANO et al., Phys. Rev. Lett., 80, 5544 (1998).

21. A. FASOLI et al., Phys. Rev. Lett., 75, 645 (1995).

22. A. FASOLI et al., Nucl. Fusion, 35, 1485 (1995).

23. M. KEILHACKER et al., Plasma Phys. Control. Fusion, 39, B1 (1997).

24. E. DOYLE et al., Phys. Fluids B, 3, 2300 (1991).

25. R. GOLDSTON et al., J. Comput. Phys., 43, 61 (1981).

26. P. R. THOMAS et al., "Heating of Thermal Ions by Alphas in the JET DT Experiment," Proc. 28th EPS Conf. Controlled Fusion and Plasma Physics, Funchal, Madeira, June 18-22, 2001, ECA Vol. 25A, 929 (2001)

27. C. Z. CHENG, L. CHEN, and M. S. CHANCE, Ann. Phys., 161, 21 (1985).

28. R. BETTI and J. P. FREIDBERG, Phys. Fluids B, 3, 1865 (1991). 29. N. N. GORELENKOV and C. Z. CHENG, Phys. Plasmas, 2, 1961 (1995). 
30. G. Y. FU and J. W. VAN DAM, Phys. Fluids B, 1, 1949 (1989).

31. R. R. METT and S. M. MAHAJAN, Phys. Fluids B, 4, 2885 (1992).

32. B. N. BREIZMAN and S. E. SHARAPOV, Plasma Phys. Control. Fusion, 37, 1057 (1995).

33. D. BORBA et al., in Theory of Fusion Plasmas (Proc. Joint VarennaLausanne Int. Workshop), Varenna, Italy, p. 327, Societa Italiana di Fisica/Editrice Compositori, Bologna, Italy (1996).

34. D. O'BRIEN et al., Nucl. Fusion, 32, 1351 (1992).

35. G. T. A. HUYSMANS, J. P. GOELDBLOED, and W. KERNER, Proc. CP90 Conf. Computational Physics, Amsterdam, the Netherlands, September 10-13, 1990, p. 371, World Scientific Publ. Co. (1991).

36. H. L. BERK et al., Phys. Plasmas, 2, 3401 (1995).

37. A. B. MIKHAILOVSKII, G. T. A. HUYSMANS, W. KERNER, and S. E. SHARAPOV, Plasma Phys. Rep., 23, 844 (1997).

38. R. NAZIKIAN et al., Phys. Rev. Lett., 78, 2976 (1997).

39. K.-L.WONG et al., Phys. Rev. Lett., 76, 2286 (1996).

40. A. MURARI et al., Plasma Phys. Control. Fusion, 47, B249 (2005).

41. A. SANTAGIUSTINA et al., Proc. 19th Symp. Fusion Technology, Lisbon, Portugal, September 16-20, 1996, Vol. 1, p. 989 (1996).

42. A. FASOLI et al., Proc. XV IAEA Int. Conf. Plasma Physics and Controlled Fusion, Sevilla, Spain (1994).

43. L. VILLARD and J. VACLAVIK, Nucl. Fusion, 37, 351 (1997).

44. A. FASOLI et al., Plasma Phys. Control. Fusion, 44, B159 (2002).

45. D. TESTA and A. FASOLI, Nucl. Fusion, 41, 809 (2001).

46. A. FASOLI, A. JAUN, and D. TESTA, Phys. Lett. A, 265, 288 (2000).

47. A. JAUN, A. FASOLI, J. VACLAVIK, and L. VILLARD, Nucl. Fusion, 40, 1343 (2000).

48. D. TESTA et al., Plasma Phys. Control. Fusion, 46, S59 (2004). 49. N. N. GORELENKOV et al., Nucl. Fusion, 45, 226 (2005).

50. C. BOSWELL et al., Bull. Am. Phys. Soc., (2005).

51. G. Y. FU et al., Phys. Plasmas, 3, 4036 (1996).

52. D. TESTA, G. Y. FU, A. JAUN, A. FASOLI, and O. SAUTER, Nucl. Fusion, 43, 479 (2003).

53. D. TESTA, C. BOSWELL, and A. FASOLI, Nucl. Fusion, 45, 907 (2005).

54. D. TESTA, A. FASOLI, and A. JAUN, Nucl. Fusion, 43, 724 (2003).

55. R. PITTS et al., J. Nucl. Mater., 337, 146 (2005).

56. W. W. HEIDBRINK and G. SADLER, Nucl. Fusion, 34, 535 (1994).

57. K. McGUIRE et al., Phys. Rev. Lett., 50, 891 (1983).

58. A. A. KOROTKOV, A. GONDHALEKAR, and A. J. STUART, Nucl. Fusion, 37, 35 (1997).

59. A. I. KISLYAKOV, A. V. KHUDOLEEV, S. S. KOZLOVSKIJ, and M. P. PETROV, Fusion Eng. Des., 34-35, 107 (1997).
60. A. BOILEAU et al., Nucl. Fusion, 29, 1449 (1989).

61. K. G. McCLEMENTS, R. O. DENDY, and A. GONDHALEKAR, Nucl. Fusion, 37, 473 (1997).

62. D. TESTA, W. G. F. CORE, and A. GONDHALEKAR, Phys. Plasmas, 6, 3498 (1999).

63. A. A. KOROTKOV and A. M. ERMOLAEV, Proc. 22nd EPS Conf. Controlled Fusion and Plasma Physics, Europhysics Conference Abstracts 19C, III-389, European Physical Society, Petit Lancy, Switzerland (1995).

64. A. A. KOROTKOV, A. GONDHALEKAR, and R. J. AKERS, Phys. Plasmas, 7, 957 (2000).

65. D. D. RYUTOV, Physica Scripta, 45, 153 (1992).

66. P. HELANDER, M. LISAK, and D. D. RYUTOV, Plasma Phys. Control. Fusion, 35, 363 (1993).

67. F. S. ZAITSEV, R. J. AKERS, and M. R. O'BRIEN, Nucl. Fusion, 42, 1340 (2002).

68. J. KÄLLNE, L. BALLABIO, J. FRENJE, S. CONROY, G. ERICSSON, M. TARDOCCHI, E. TRANEUS, and G. GORINI, Phys. Rev. Lett., 86, 1246 (2000).

69. L. BALlABIO, G. GORINI, and J. KÄLlNE, Phys. Rev. E, 55, 3358 (1997).

70. G. J. SADLER et al., Fusion Technol., 18, 556 (1990).

71. L.-G. ERIKSSON et al., Nucl. Fusion, 29, 87 (1989).

72. J. JACQUINOT and G. J. SADLER, Fusion Technol., 21, 2254 (1992).

73. O. N. JARVIS et al., Nucl. Fusion, 36, 1513 (1996).

74. V. G. KIPTILY et al., Nucl. Fusion, 42, 999 (2002).

75. M. J. MANTSINEN et al., Phys. Rev. Lett., 89, 115004 (2002).

76. V. G. KIPTILY et al., Proc. 33rd EPS Conf. Plasma Physics and Controlled Fusion, Rome, Italy, ECA Vol. 30I, p. 1,077 (2006).

77. V. G. KIPTILY et al., Nucl. Fusion, 45, L21 (2005).

78. V. G. KIPTILY, F. E. CECIL, and S. S. MEDLEY, Plasma Phys. Control. Fusion, 48, R59 (2006).

79. V. G. KIPTILY et al., Tech. Phys., 43, 471 (1998).

80. V. G. KIPTILY et al., Phys. Rev. Lett., 93, 115001 (2004).

81. S. E. SHARAPOV et al., Phys. Plasmas, 9, 2027 (2002).

82. A. C. C. SIPS and G. J. KRAMER, Plasma Phys. Control. Fusion, 35, 743 (1993).

83. H. L. BERK et al., Phys. Rev. Lett., 87, 185 (2002).

84. A. D. BEKLEMISHEV and W. HORTON, Phys. Fluids B, 4, 200 (1992).

85. E. JOFFRIN et al., Nucl. Fusion, 43, 1167 (2003).

86. S. E. SHARAPOV et al., Nucl. Fusion, 46, S868 (2006).

87. S. E. SHARAPOV et al., Nucl. Fusion, 45, 1168 (2005).

88. D. DARROW et al., Rev. Sci. Instrum., 75, 3566 (2004).

89. S. BAEUMEL et al., Rev. Sci. Instrum., 75, 3563 (2004).

90. D. DARROW et al., Rev. Sci. Instrum., 77, 10E701 (2006). 